
3 Research Square
Preprints are preliminary reports that have not undergone peer review.
They should not be considered conclusive, used to inform clinical practice, or referenced by the media as validated information.

\title{
Circumscription and typification of sphagnicolous omphalinoid species of Arrhenia (Hygrophoraceae) in Newfoundland and Labrador: three obligate and one facultative species
}

\author{
Andrus Voitk ( $\sim$ seened@gmail.com ) \\ Foray Newfoundland \& Labrador https://orcid.org/0000-0002-3483-8325 \\ Irja Saar \\ Institute of Ecology and Earth Sciences, University of Tartu: Tartu Ulikool \\ Bibiana Moncada \\ Universidad Distrital Francisco Jose de Caldas \\ Edgar B. Lickey \\ Department of Biology, Bridgewater College
}

\section{Research Article}

Keywords: Arrhenia oniscus, Arrhenia sphagnicola, Omphalina rivulicola, bog agarics, bog Arrhenia. Introduces 2 new taxa and 5 new typifications

Posted Date: February 11th, 2022

DOI: https://doi.org/10.21203/rs.3.rs-1336756/v1

License: @ (i) This work is licensed under a Creative Commons Attribution 4.0 International License. Read Full License 


\section{Abstract}

Molecular studies of sphagnicolous arrhenias in Newfoundland and Labrador created four clades in Arrhenia, three obligate sphagnophiles (two scaly-capped and one smooth-capped) and one facultative (smooth-capped). Nomenclatural review recovered 16 names applied to omphalinoid sphagnicolous taxa in the past. Critical review of these left five as suitable to this group. One scaly obligate sphagnophilic clade contained the type for Arr. gerardiana and the other the type for Clitocybe gerardiana var. fusca; the latter we introduce as the novel species Arr. bigelowii. It differed from the first by longer spores and a darkening reaction in $10 \%$ of collections. The smooth-capped third obligate sphagnophilic clade contained the types of Ag. telmatiaeus and Omphalina fusconigra; we recombined it as Arr. telmatiaea. This is the darkest species of the group, with a more northern distribution. The facultative sphagnophile was identified as Arr. philonotis, a lighter smooth-capped species also with a more northern distribution. In addition, we collected an unidentified smooth-capped facultatively sphagnophilic species of Omphalina of the $O$. pyxidata complex. All five species are distributed in both Europe and North America. We describe each species of Arrhenia with a sequenced type, providing new type material where needed, and add an informal description of the Omphalina. Overall, this study adds new sequences from 82 specimens of sphagnicolous arrhenias to the two existing in GenBank when we began, 11 new sequences of the unidentified species of Omphalina, and several other arrhenias.

\section{Introduction}

\section{Aim}

This study was undertaken to identify the sphagnicolous omphalinoid species of Arrhenia Fr. native to the Canadian province of Newfoundland and Labrador (NL). Our interest to learn more about this group was stimulated in part because over 15 years one of these species has been the logo for Foray Newfoundland \& Labrador (FNL), the mushroom club of the province, with which three of the four authors are or have been associated.

\section{Definitions}

Arrhenia: a genus erected by Fries (1849), belonging to the Hygrophoraceae (Lodge et al. 2014). Currently it contains species of bryophilous fungi with basiomata varying from pleurotelloid to omphalinoid, pilei varying from nutant to infundibuliform, hymenia varying from reduced (flat, wavy, wrinkled or folded) to gilled, and stipes varying from absent, reduced or rudimentary, to fully developed, placed laterally, eccentrically or centrally, all particularly well represented in arctoalpine habitats. Obligate sphagnophile: taxon found only with Sphagnum L., never separately. Facultative sphagnophile: taxon found in Sphagnum with some regularity, but also among other mosses, usually in barren locations where Sphagnum is present. Sphagnicolous: taxa regularly found in Sphagnum, whether in an obligate or facultative relationship. Our definition excludes species normally preferring other habitats, even if found on occasion in Sphagnum, presumably by chance (e. g. an occasional Craterellus Pers., or a lignicolous species on wood that happens to be in Sphagnum). Omphalinoid: agaric species with small basiomata having an umbilicate pileus, decurrent gills and central stipe.

\section{History of nomenclatural usage}

Earlier treatments of species we now consider omphalinoid sphagnicolous arrhenias applied epithets inconsistently, using several names for what seems like a small number of taxa. Increasing consistency in both morphologic species concepts and nomenclature began to emerge during the last 75 years. Eleven major publications by 12 authors who have collected and studied these species (i.e. excluding check lists and general reviews or descriptions of regional 
mycota), use eight names between them to describe three to four different morphospecies: Favre $(1948)-3$, Redhead (1979) - 2, Clémençon (1982) - 4, Lange \& Lange (1982) - 2, Bigelow (1958, 1985) - 5, Kuyper (1995) - 3, Breitenbach \& Kränzlin (1991) - 2, Bon (1997) - 4, Gminder (2001) - 1, and Elborne (2012) - 3. Undoubtedly Jules Favre, father of alpine mycology and mycoecology (Brunner et al. 2017), provided the pivot point to a more coherent and unified taxonomy and nomenclature of sphagnicolous arrhenias. Regular collecting in subalpine peat bogs, followed by seven seasons of daily collecting trips to the alpine zone of the Swiss National Park gave him unsurpassed familiarity with these species in their environment. His work (Favre 1948, 1955) became the cynosure for this mycota, inspiring and influencing subsequent investigators for a long time. With the obvious expertise of his factual observations, came occasional nomenclatural conventions based on less accurate influences. These in no way lessened the enormous contribution of Favre, but did propagate some nomenclatural problems in this small group. Below we review names used for omphalinoid sphagnicolous arrhenias by year of publication, taken from the above ten major publications or their authors, as well as names, synonyms and citations found in the pertinent protologues-adding a few from diverse sources-and assess the suitability of each to sphagnicolous arrhenias.

\section{Review of taxonomy}

1. 1782. Agaricus tigrinus Bull.,nom. sanct. Described as a white, lignicolous species (Buillard 1782) and later recombined by Fries (1818) as Lentinus tigrinus (Bull.) Fr., nom. sanct., the name by which we know it today. This white wood dweller does not fit a grey-brown, sphagnicolous Arrhenia, and was not considered a sphagnicolous Arrhenia by author or sanctioner. It is listed here because taxonomic confusion has caused some of its homoand synonyms to be so considered.

2. 1794. Agaricus epichysium Pers.,nom. sanct. Described as growing on hollow willow trunks (Persoon 1794) this species is currently known as the wood-dwelling Arrhenia epichysium (Pers.) Redhead, Lutzoni, Moncalvo \& Vilgalys. Because rotten wood can also be found in bogs, lignicolous fungi have been collected from Sphagnum (Bunyard et al. 2008). Possibly because of such chance association the name "epichysium" has been applied to some of these species on occasion, persisting as late as 1982 (Lange \& Lange 1982). Currently Arr. epichysium is not considered a sphagnicolous species and we do not consider the name suitable for sphagnicolous arrhenias.

3. 1801. Agaricus epichysium var. icmadophilus Pers., nom. sanct. In translation, Persoon's (1801) entire description, omitting measurements, reads: "very delicate, pileus funnel-shaped and dark grey, gills planodecurrent and grey. Arising in turf among Sphagnum." Initially Bigelow (1958) preferred this laconian description, sanctioned by Fries (1821), over the use of the light-coloured "oniscus" for the dark sphagnicolous Arrhenia species, and transferred it to Clitocybe, raising it to species level. Later Bigelow (1985) gave up icmadophilus in favour of oniscus, because he felt that the description of $\mathrm{Ag}$. epichysium var. icmadophilus lacked specific identifying features, and "is very short and could also be of several species". In his doctoral thesis Redhead (1979) followed Bigelow, but states (personal communication, 2018) that by 2002 he had "acquiesced to standard usage". Apart from these brief but transient uses, the epithet has never been in common usage and has not been applied to this group. We find nothing convincing in the scant protologue of $A$. epichysium var. icmadophilus that compels us to apply the name to any species of sphagnicolous Arrhenia with conviction, and in view of the absence of type material, paucity of detail in the protologue and lack of continued usage, we are content to follow Bigelow and Redhead in this matter.

4. 1805. "Agaricus tigrinus Alb. \& Schw." Fries $(1818,1821)$ cites these authors' publication while describing Agaricus affricatus Fr. This may have confused some workers into thinking the description of Ag. tigrinus by von Albertini \& von Schweinitz (1805) is the protologue for a new species, something claimed by neither Fries nor von Albertini \& von Schweinitz. The latters' Conspectus is a treatment of regional mycota, made up of both new and previously known species. They mark all new species "nobis" (Latin for "from or by us", in the sense of described 
as new by us) and provide a list of the new species they describe (Catalogus fungorum novorum nostratium, $\mathrm{P}$ XIII). Agaricus tigrinus is not marked "nobis" and does not appear in the list, making it clear that von Albertini \& von Schweinitz did not introduce it as a new species. The title of their book states "e metodo Persooniana" to indicate that they refer to Persoon's work. Thus, in this case they refer to Persoon's 1801 description of Agaricus tigrinus. In turn, Persoon also did not proffer his description as that of a new species, but cites earlier descriptions by Sowerby and by Bulliard. With the latter citation we have come full circle to the first taxon in this list, indicating why it needed to be included. "Agaricus tigrinus Alb. \& Schw." does not exist and cannot be used.

5. 1818. Agaricus affricatus Fr., nom. sanct. This epithet is occasionally (mis)applied to sphagnicolous arrhenias, probably because Fries (1818) described it as common in swamps (copiose in paludibus). No doubt the description of an omphalinoid agaric in swamps, with darkening (nigricantibus), grey, bristly scales (squamulis pilosis cineris), may suggest a similarity to scaly-capped arrhenias, but Fries clearly described Ag. affricatus as a white-capped mushroom, "albidus" in the protologue (Fries 1818) and "albido" in his sanctioning Systema (Fries 1821). He did not change this description during his lifetime, and repeated it later with a coloured illustration (Fries 1867), which, he states fits (adumbrat) his Ag. affricatus. The illustration shows a basidioma with a snowwhite, non-striate pileus with black scales, resembling the current Lentinus tigrinus. Both description and image are unsuitable to represent grey-brown and translucent sphagnicolous arrhenias.

6. 1818. Agaricus incomtus Fr.,nom. sanct. Described as sphagnicolous by Fries (1818), but to our knowledge, hitherto not applied to a species of Arrhenia. Fries described the cap and stem as grey and the gills as dirty white, yellowing with time. Unlike our sphagnicolous arrhenias, the gills are adnate, not decurrent. Fries also cited varieties rimosa and truncigena of Agaricus discolor, as described by von Albertini \& von Schweiniz. These authors described the former as a woodland species and the latter as lignicolous, reinforcing that this epithet, possibly encompassing several current taxa, is not applicable to sphagnicolous species of Arrhenia.

7. 1818. Agaricus oniscus Fr., nom. sanct. Both in his protologue (Fries 1818) and sanctioning description (Fries 1821) of this species, Fries leaves no doubt that he is providing a new name for Ag. cespitosus Bolton (1788). A recent review (Voitk 2021) of the protologues and associated original material of both determined that $\mathrm{Ag}$. cespitosus was a later synonym for the species currently known as Lichenomphalia umbellifera, and because Fries had declared $\mathrm{Ag}$. oniscus a synonym for Ag. cespitosus, Ag. oniscus also becomes a later synonym for that taxon. The name, therefore, is both unsuitable and unavailable for sphagnicolous arrhenias.

8. 1828. Agaricus philonotis Lasch. Lasch (1828) described this species, currently known as Arrhenia philonotis_(Lasch) Redhead, Lutzoni, Moncalvo \& Vilgalys, from bogs near Brandenburg, a basidioma with an umbilicate cap and deeply decurrent, whitish, subdistant gills. He described the cap as sallow (lurido), light grey (subcinereus), and sparsely wooly (leviter tomentosus). Qualifiers in formal descriptions are used to distinguish the character qualified from its "normal" state; of the two, the qualifier becomes the more important descriptor. If Lasch felt it necessary to add "leviter", it was to stress the sparse nature of the cap ornamentation, to distinguish it from species with truly tomentose caps. Even should one consider "leviter" a relative concept, and dismiss "lightly, slightly, sparsely" or "minutely" as subjective, there should be no misunderstanding the meaning of "tomentosus". The term is specifically used to indicate woolly, fuzzy, fine hair, and not scales; the latter would require terms like "scabrosus" or "squamosus". Lasch's description of Ag. philonotis is compatible with smoothcapped sphagnicolous arrhenias.

9. 1836. Agaricus sphagnicola Berk. The use of this name for sphagnicolous arrhenias has been very confusing. Because Berkeley (1836) described the cap of Ag. sphagnicola as "minutely squamulose", many workers have applied the epithet to scaly-capped sphagnicolous arrhenias, while others, like Redhead (1979) in his original report, and Kuyper (1995) found that Berkeley's description, according to Kuyper, "seems to fit better for Phytoconis ericetorum" (= L. umbellifera). A recent detailed analysis of Berkeley's protologue (Voitk 2021) 
supported the early opinion of Redhead (1979), and agreed with Kuyper (1995) that Berkeley's protologue fit with L. umbellifera, but produced significant conflicts, if applied to sphagnicolous arrhenias. For nomenclatural stability the species was neotypified with a specimen collected and identified by Berkeley as Ag. sphagnicola, and subsequently identified by Redhead (1979) as Gerronema ericetorum (= L. umbellifera), making this unsuitable name unavailable for sphagnicolous arrhenias.

10. 1865. "Agaricus affricatus Berk. \& Broome”. Berkeley \& Broome (1865) applied Ag. affricatus Fr. (see beginning of this list) to a different species by mistake. Because the name was already in use within that genus, its use for another species was illegitimate, making it unavailable for sphagnicolous arrhenias.

11. 1873. Agaricus gerardianus Peck. Along with a coloured aquarelle, Peck (1873) described a small, brown mushroom with a cap that was "rough with scattered blackish points", growing in "sphagnous marshes" of New York State. This is the first unambiguous description of a truly scaly-capped sphagnicolous omphalinoid species, clearly shown on the illustration. In the margin of his early hand-written notes Peck wrote, "Is it Ag. affricatus," indicating that he actively considered earlier and equally scaly descriptions, before concluding that this was a new species. It has been transferred to Arrhenia (Elborne 2008), and is currently known as Arrhenia gerardiana (Peck) Elborne. The description fits scaly-capped sphagnicolous arrhenias.

12. 1883. Agaricus telmatiaeus Berk. \& Cooke. This name appeared with the reproduction of an aquarelle by George Massee of his collection near Scarborough, in volume 2 of Cooke's "Illustrations of British Fungi (Hymenomycetes)". The Illustrations appeared in installments, and Ag. telmatiaeus, plate 240, appeared AprMay, 1883 (Stefleu \& Cowan 1976). In addition to illustrating the taxon, Massee's watercolour contained separate analytic figures (figures in addition to those illustrating the species generally, demonstrating details to aid identification), e.g. depictions of spores, gill shape, tiers of lamellulae, stem context and content, etc. The "Handbook of British Fungi", for which the Illustrations were meant to be an atlas, also appeared in parts; the segment treating Ag. telmatiaeus (Cooke 1885) appeared Jan-Apr, 1885 (Stefleu \& Cowan 1976). Although the synopsis appeared two years after the name, the name was valid on publication, because the International Code of Nomenclature for algae, fungi, and plants (henceforth the Code; Turland et al 2018) accepts an illustration and figure with analysis in lieu of a synopsis for taxa published before 1908. Berkeley \& Cooke cited Cooke's 1871 treatment of Ag. affricatus Berk. \& Brooke, to indicate this was the same species, but because they provided a new name, a new synopsis, a new illustration, and a new type specimen, they made it clear that they were publishing the new name as a new species, not a replacement name for the old type specimen. That this was their intent is confirmed by an annotation, "Type", on the herbarium sheet of Massee's collection from Scarborough, and "from type" on Massee's aquarelle depicting the same collection. Dennis (1948) reviewed Ag. telmatiaeus and noted the confusion of names, authors, collections and illustrations. He concluded that Massee's collection from Scarborough was the type specimen for $\mathrm{Ag}$. telmatiaeus. The description of this taxon is compatible with a dark smooth-capped sphagnoicolous species of Arrhenia.

13. 1883. Agaricus telmaticus Cooke. The name appeared as entry 1999 in Berkeley and Broome's Notices of British fungi in late 1883, a review of noteworthy finds and publications, which states, " $A$. (Omphalia) telmaticus, Cooke, tab. 240. On Sphagnum. This is our A. affricatus, which appears not to be the plant of Fries." Placing the period after the plate number, rather than after Cooke, Berkeley and Broome notify the reader that entry 1999 reports a new taxon illustrated on a specific plate (240) in an atlas published by Cooke, not a new species described by Cooke. Failure to recognize this has led to the misinterpretation that Berkeley and Brooke meant to state that Cooke was the author of a taxon named Ag. telmaticus. Cooke has never published such an epithet. The cited plate 240 in Cooke's Illustrations shows Ag. telmatiæus, clearly attributing authorship to Berkeley and Cooke. Both epithets cite Ag. affricatus as a synonym. The epithet "telmaticus", used by Berkeley and Brooke, is a correct inflectional form of the validly published earlier name for the same species, considered an orthographic variant 
by the Code, which rules that the first validly published correct variant be accepted. Were they considered separate names rather than orthographic variants, "telmaticus" would be a superfluous name, to be rejected in favour of the earlier name. Either way, this name is not not available.

14. 1883. Agaricus peculiaris Britz. Apart from giving a fitting size, the entirety of Britzelmayr's (1883) very sparse protologue is that his new bog species has grey, moderately spaced gills with spores $11-14 \times 4 \mu \mathrm{m}$, and is near Ag. oniscus. He does not state whether the cap is umbilicate or plane, smooth or scaly, or whether the gills are decurrent or adnate. An illustration shows a grey-black mushroom with a smooth, black-brown, deeply infundibuliform cap with decurrent gills. Comparison of other known scaly-capped species illustrated by Britzelmayr showed that he illustrated scaly caps faithfully. Illustrated spores are doubly curved with a Q value 3.4-4.5 (Average 3.8). None of our smooth-capped sphagnicolous arrhenias have spores even close to this size; only one scaly-capped species has spores in this length, but not as prominently doubly curved and with and average $Q$ of 2.3, and no $Q$ value above 3.3. The image does not fit with any of our species either macroscopically or microscopically. In an effort to redefine taxa described by Britzelmayr, Bresinsky \& Strangl (1974) explored the type locality and identified what they believed to be the same species as Omphalina sphagnicola (Berk.) M.M. Moser, based on comparison with a non-type collection by Britzelmayr, made 15 years after the protologue. Bersinsky \& Strangl provide no descriptive information about either specimen. Unable to fit the original material to a sphagnicolous Arrhenia, we do not consider the name suitable for this group.

15. 1958. Clitocybe gerardiana var. fusca Bigelow. The effort to consider white-spored fungi with funnel-shaped caps as members of Clitocybe presented Bigelow an opportunity to review several species of Omphalina, including many that have since been transferred to Arrhenia. On reassigning Peck's Agaricus gerardianus to Clitocybe, Bigelow encountered some darker specimens, seemingly limited to a small locality, microscopically indistinguishable from the more widespread lighter specimens. In a later review of Clitocybe Bigelow (1985) states that he first described this entity as Clitocybe sphagnorum Bigelow, "nom. prov.", in his PhD dissertation. However, when he came to describe it formally he decided it was more aptly ranked as a variety, and named it Clitocybe gerardiana var. fusca Bigelow (Bigelow 1958). He described it as a scaly-capped sphagnicolous species with dark caps, squamulose in the middle and smooth at the periphery. After publication of the variety, he became misled to believe that it was the same entity as Omphalina fusconigra Orton, and synonymized his variety with Orton's species. Bigelow's description remains suitable for scaly-capped sphagnicolous arrhenias.

16. 1960. Omphalina fusconigra Orton. Orton (1960) was unable to find an existing "description which fit this rather characteristic sphagnicolous agaric", which differed "from the other sphagnicolous species of Omphalina in consistently darker blackish brown cap and stem..." To Orton the main macroscopic character worthy of note, which readily distinguishes this species from other sphagnicolous arrhenias, is the dark, black-brown colouring, not cap texture. He described the cap as "sometimes slightly rugulose or scurfy-flocculose at centre", in other words an irregularity that was the exception, minimal, and limited. Both wrinkled (rugulose) and flat surfaces can be scaly, scurfy-flocculose, or smooth. Probably because scaly-capped arrhenias are commonly encountered in bogs, the mention of any cap irregularity, no matter how uncommon, limited or inconsequential, encouraged misinterpretation, and $O$. fusconigra became considered a scaly-capped species, synonymized with $\mathrm{Ag}$. sphagnicola (also not described as scaly!) and subsequently with the truly scaly Ag. gerardianus. We conclude that this name, currently Arrhenia fusconigra (P. D. Orton) P.A. Moreau \& Courtec., is suitable to apply to dark smooth-capped sphagnicolous arrhenias.

In summary, from a field of 16 potential candidates we identified five epithets that fit the species we have observed in our bogs, two with clearly scaly caps (Ag. gerardianus, and $C$. gerardiana var. fusca) and three with primarily smooth caps, albeit keen observation may reveal some irregularity or fine surface ornamentation at times (Ag. philonotis, Ag. telmatiæus, and O. fusconigra).

Page 6/45 


\section{Materials And Methods}

Type collections. Peck (1873) listed two collections in his protologue of Ag. gerardianus, but did not specify either as type; only one of the two syntypes could be located. All four basidiomata (NYSf 1339.1-4) of this collection were examined, but their condition was not sufficiently robust to permit sequencing. Two attempts to find fresh collections around the type locality near Sand Lake, NY, were unsuccessful, so that one of Peck's contemporaneous collections from Essex Co., NY, identified by him as Ag. gerardianus, was selected (NYSd 4725). One basidioma (NYSd 4725.1) from this was used for both DNA extraction and microscopy. The spore size of NYSd 4725.1 matched that of all four basidiomata in Peck's syntype, NYSf 1339.1-4. The type collection for Clitocybe gerardiana var. fusca, collected by A.H. Smith, contains over 30 basidiomata. From that colletions $\mathrm{MICH}$ made a gift of a fragment consisting of approximately $1 / 2$ pileus with gills attached, which was used for both DNA extraction and microscopy. The remaining material from this was deposited in TUF (TUF117871). One basidioma from the type collections of Ag. telmatiaeus, and one from $O$. fusconigra were sequenced successfully.

Other collections. Study collections were selected from over 150 collections identified to be sphagniocolous omphalinoid arrhenias in the private herbaria of Andrus Voitk and FNL, collected with normal field techniques and airdried at $20-30^{\circ} \mathrm{C}$. Selection attempted to incorporate samples of all potential "species" and to represent a wide distribution in NL. In addition, nine collections from an archipelago near Saint-Augustin, Québec, on the north shore of the St Lawrence Basin, a similar area contiguous with the southern Labrador coast, were included. Specimens not associated with Sphagnum were included if Sphagnum was nearby and collections of similar sphagnicolous specimens had been made. Three collections from mossy grassland with no nearby Sphagnum were added to compare to similar specimens collected in Sphagnum.

Of 64 selected regional collections, sequences were obtained from 60 . Two were excluded after analysis because they were unidentified singleton species from a distant portion of the Arrhenia tree for which we had no photo and inadequate habitat or substrate data, leaving 58 sequenced collections, which make up the regional target material for this study (51 from NL, seven from QC; 36 from Voitk, 22 from FNL). In addition, 35 extraregional collections of sphagnicolous arrhenias from various herbaria were sequenced for this study, to which were added two GenBank sequences of sphagnicolous arrhenias available at the time. Herbaria are designated with Index Herbariorum codes (Thiers 2021).

Macroscopic examination. NL specimens were photographed in situ and macroscopic descriptions were based on fresh specimens. Apart from capsule descriptions of types, only personally observed sequence-identified regional specimens were used for taxonomic descriptions.

Microscopic examination. Light microscopic observations (Zeiss 392560 with Apo 100/1.25), were conducted at 1000 $\times$ magnification (oil immersion), using $2 \% \mathrm{KOH}$. Spore length and width were measured to $0.5 \mu \mathrm{m}$ accuracy; measurements deviating from $0.5 \mu \mathrm{m}$ increments are due to calculation of an objective correction factor. A minimum of 20 spores per single basidioma was used to calculate average values. Statistical analysis of spore sizes was done with a Student's t-test (two-tail, two-sample assuming equal variances) using the data analysis package in Microsoft Excel 2013.

DNA extraction and nuclear sequencing was done in three laboratories. DNA extraction at Bridgewater College (sequences marked with * in Table 1) followed Lickey (2003). DNA extraction at The Field Museum (sequences without a UDB code and not marked with * in Table 1) followed Sulzbacher et al. 2016). DNA extraction at Tartu University (UDB code in Table 1) followed Saar \& Voitk (2015). 
Table 1

Details of sequenced specimens in order of appearance in Fig. 1, excluding the single NL species in the Omphalina pyxidata complex (shown in Tab. 2), and the sphagnicolous arrhenias (shown in Tab. 3)

\begin{tabular}{|c|c|c|c|c|}
\hline $\begin{array}{l}\text { Species } \\
\text { (as recorded on } \\
\text { GenBank } \\
\text { deposit) }\end{array}$ & $\begin{array}{l}\text { GB code above, } \\
\text { UNITE code } \\
\text { below }\end{array}$ & $\begin{array}{l}\text { Primary Herbarium } \\
\text { number above, copy } \\
\text { below }\end{array}$ & $\begin{array}{l}\text { COUNTRY } \\
\text { (Province for } \\
\text { Canada) }\end{array}$ & Reference \\
\hline $\begin{array}{l}\text { Omphalina } \\
\text { rivulicola }\end{array}$ & U66451 & & & Lutzoni 1997 \\
\hline $\begin{array}{l}\text { Omphalina } \\
\text { pyxidata }\end{array}$ & U66450 & & & Lutzoni 1997 \\
\hline $\begin{array}{l}\text { Omphalina } \\
\text { pyxidata }\end{array}$ & UDB0799020 & TUF114379 & ESTONIA & this study \\
\hline $\begin{array}{l}\text { Omphalina cf. } \\
\text { rivulicola }\end{array}$ & HQ445617 & & SVALBARD & Bjorbaekmo et al. 2010 \\
\hline $\begin{array}{l}\text { Omphalina cf. } \\
\text { rivulicola }\end{array}$ & GU234117 & & SVALBARD & Geml et al. 2012 \\
\hline $\begin{array}{l}\text { Omphalina } \\
\text { chionophylla }\end{array}$ & GU234144 & & SVALBARD & Geml et al. 2012 \\
\hline $\begin{array}{l}\text { Omphalina } \\
\text { chionophylla }\end{array}$ & MH862276 & & SVALBARD & Vu et al. 2019 \\
\hline $\begin{array}{l}\text { Arrhenia } \\
\text { epichysium }\end{array}$ & KC237880 & LE262961 & RUSSIA & Zvyagina et al. 2015 \\
\hline $\begin{array}{l}\text { Arrhenia } \\
\text { epichysium }\end{array}$ & U66442 & & & Lutzoni 1997 \\
\hline $\begin{array}{l}\text { Arrhenia } \\
\text { sphagnicola }\end{array}$ & GB XXXXXXXX & DAOMXXXXXX & CANADA, NL & this study \\
\hline $\begin{array}{l}\text { Arrhenia } \\
\text { velutipes }\end{array}$ & UDB034634 & TUF117593 & CANADA, NL & this study \\
\hline $\begin{array}{l}\text { Arrhenia } \\
\text { philonotis }\end{array}$ & UDB024403 & M-0155052 & GERMANY & this study \\
\hline $\begin{array}{l}\text { Arrhenia } \\
\text { sphagnicola }\end{array}$ & GB XXXXXXXX & DAOMXXXXXX & CANADA, NL & this study \\
\hline $\begin{array}{l}\text { Arrhenia } \\
\text { obscurata }\end{array}$ & $\begin{array}{l}\text { MT967333 } \\
\text { UDB024573 }\end{array}$ & TUF117230 & CANADA, NL & Voitk et al. 2020 \\
\hline $\begin{array}{l}\text { Arrhenia } \\
\text { obscurata }\end{array}$ & МТ998930 & voucher lost & CANADA, NL & Voitk et al. 2020 \\
\hline $\begin{array}{l}\text { Arrhenia } \\
\text { acerosa }\end{array}$ & $\begin{array}{l}\text { MT967312 } \\
\text { UDB032044 }\end{array}$ & UPS-F151993 & SWEDEN & Voitk et al. 2020 \\
\hline $\begin{array}{l}\text { Arrhenia } \\
\text { svalbardensis }\end{array}$ & $\begin{array}{l}\text { MT967358 } \\
\text { UDB024589 }\end{array}$ & $0-50446$ & SVALBARD & Voitk et al. 2020 \\
\hline $\begin{array}{l}\text { Arrhenia } \\
\text { auriscalpium }\end{array}$ & UDB037768 & 0-304914 & NORWAY & $\begin{array}{l}\text { sequenced as part of the } \\
\text { NorBOL project; no associated } \\
\text { publication }\end{array}$ \\
\hline
\end{tabular}




\begin{tabular}{|c|c|c|c|c|}
\hline $\begin{array}{l}\text { Species } \\
\text { (as recorded on } \\
\text { GenBank } \\
\text { deposit) }\end{array}$ & $\begin{array}{l}\text { GB code above, } \\
\text { UNITE code } \\
\text { below }\end{array}$ & $\begin{array}{l}\text { Primary Herbarium } \\
\text { number above, copy } \\
\text { below }\end{array}$ & $\begin{array}{l}\text { COUNTRY } \\
\text { (Province for } \\
\text { Canada) }\end{array}$ & Reference \\
\hline $\begin{array}{l}\text { Arrhenia } \\
\text { auriscalpium }\end{array}$ & U66428 & $\begin{array}{l}\text { Lutzoni 930804-5, } \\
\text { DUKE \& } 0\end{array}$ & & Lutzoni 1997 \\
\hline $\begin{array}{l}\text { Arrhenia } \\
\text { elegans }\end{array}$ & JF908757 & MCVE-16455 & ITALY & Osmundson et al. 2013 \\
\hline $\begin{array}{l}\text { Arrhenia } \\
\text { subglobispora }\end{array}$ & KR606032 & & SWITZERLAND & $\begin{array}{l}\text { Senn-Irlet, direct submission } \\
\text { (2015) }\end{array}$ \\
\hline $\begin{array}{l}\text { Arrhenia } \\
\text { retiruga }\end{array}$ & МТ967340 & TUF117227 & ESTONIA & Voitk et al. 2020 \\
\hline $\begin{array}{l}\text { Arrhenia } \\
\text { retiruga }\end{array}$ & UDB036702 & $0-21925$ & NORWAY & $\begin{array}{l}\text { sequenced as part of the } \\
\text { NorBOL project; no associated } \\
\text { publication }\end{array}$ \\
\hline Arrhenia lobata & MT967332 & $\begin{array}{l}\text { DAOM981256 } \\
\text { TUF117633 }\end{array}$ & CANADA, NL & Voitk et al. 2020 \\
\hline Arrhenia lobata & U66429 & $\begin{array}{l}\text { Lutzoni \& Lamoure } \\
\text { 910824-1, DUKE }\end{array}$ & FRANCE & Lutzoni 1997 \\
\hline
\end{tabular}

The sequences were inspected and assembled using Sequencher 5.4 software (Gene Codes, Ann Arbor, USA), and uploaded into PlutoF cloud database (Abarenkov et al. 2010b), including the collection data, partly reachable through the public web output UNITE (http//unite.ut.ee; Abarenkov et al. 2010a). Nucleotide sequence data were deposited in GenBank (see Tabs 1-3).

Phylogenetic analysis. The alignments were performed using L-INS-i strategy as implemented in MAFFT v7.475 (Katoh and Standley 2013). Minor manual adjustments were performed with SeaView 4.7 (Gouy et al 2010). Bayesian inference of phylogeny was performed with MrBayes 3.2.6 (Ronquist et al 2012) with default values, the first 100K generations without reaching a stable likelihood score were discarded.

Maximum likelihood (ML) analysis was performed with RAxML-HPC BlackBox v.8.2.9 (Stamatakis 2014), at the Cipres Science Gateway (Miller et al 2010; http://www.phylo.org/). Analyses deposited in TreeBase, http://purl.org/phylo/treebase/phylows/study/TB2:S29346.

\section{Results}

The specimens studied are summarized in three tables. Tab. 1 gives details of collections whose sequences make up the phylogenetic tree in Fig. 1, excluding an unidentified species in the Omphalina pyxidata complex and the sphagnicolous arrhenias. Data for collections of the unidentified species in the Omphalina pyxidata complex examined for this study, whether shown in Fig. 1 or not, are summarized in Tab. 2, and data for the sphgagnicolous arrhenias examined for this study, including those used to make up the phylogenetic trees in Figs. 1 and 2, are shown in Tab. 3. 
Table 2

Specimens of the single NL species in the Omphalina pyxidata complex (OPCUS ${ }^{\mathrm{a}}$ ) examined ${ }^{\mathrm{b}}$

\begin{tabular}{|c|c|c|c|c|c|}
\hline $\begin{array}{l}\text { CODE: } \\
\text { GenBank } \\
\text { above, } \\
\text { UNITE } \\
\text { below }\end{array}$ & $\begin{array}{l}\text { COUNTRY, STATE/PROVINCE, Town, } \\
\text { locality above, } \\
\text { Coordinates (degree decimal); } \\
\text { elevation (m asl) below }\end{array}$ & $\begin{array}{l}\text { Habitat } \\
\text { above, } \\
\text { Substrate } \\
\text { below }\end{array}$ & $\begin{array}{l}\text { Date } \\
\text { DD- } \\
\text { Mon- } \\
\text { YY }\end{array}$ & $\begin{array}{l}\text { Collector } \\
\text { above } \\
\text { Collection } \\
\text { code below }\end{array}$ & $\begin{array}{l}\text { Herbarium } \\
\text { code: } \\
\text { Main above, } \\
\text { copy below }\end{array}$ \\
\hline $\begin{array}{l}\text { MH237678 } \\
\text { c } \\
\text { UDB032178 }\end{array}$ & $\begin{array}{l}\text { CANADA, NL, Pasadena, Pasadena } \\
\text { Ski Club, clubhouse lawn } \\
48.984177 N, 57.580194 W ; 171\end{array}$ & $\begin{array}{l}\text { grassland } \\
\text { moss }\end{array}$ & $\begin{array}{l}24- \\
\text { Sep- } \\
13\end{array}$ & $\begin{array}{l}\text { Henry Mann } \\
\text { 13.09.24.av03 }\end{array}$ & $\begin{array}{l}\text { DAOM744407 } \\
\text { TUF117362 }\end{array}$ \\
\hline$M H 569861^{d}$ & $\begin{array}{l}\text { CANADA, NL, Flowers Cove } \\
51.288142 \mathrm{~N}, 56.740856 \mathrm{~W} ; 1\end{array}$ & $\begin{array}{l}\text { grass } \\
\text { moss }\end{array}$ & $\begin{array}{l}21- \\
\text { Sep- } \\
12\end{array}$ & $\begin{array}{l}\text { Andrus Voitk } \\
\text { 12.09.21.av01 }\end{array}$ & $\mathrm{n} / \mathrm{a}^{\mathrm{e}}$ \\
\hline JF908501 f & ITALY & & & & \\
\hline $\begin{array}{l}\text { MH237679 } \\
\text { c } \\
\text { UDB032179 }\end{array}$ & $\begin{array}{l}\text { CANADA, NL, Pasadena, Pasadena } \\
\text { Ski Club, clubhouse lawn } \\
48.984177 N, 57.580194 W ; 171\end{array}$ & $\begin{array}{l}\text { grassland } \\
\text { moss }\end{array}$ & $\begin{array}{l}14- \\
\text { Oct- } \\
07\end{array}$ & $\begin{array}{l}\text { Henry Mann } \\
\text { 14.10.07.av01 }\end{array}$ & $\begin{array}{l}\text { DAOM744408 } \\
\text { TUF117363 }\end{array}$ \\
\hline$\underset{f}{\mathrm{MH}} \mathrm{H} 73375$ & $\begin{array}{l}\text { CANADA, NL, Labrador, Forteau, } \\
\text { Overfall Brook trail } 51.454297 \mathrm{~N}, \\
56.947339 W ; 51\end{array}$ & $\begin{array}{l}\text { heath } \\
\text { Sphagnum } \\
\& \text { moss }\end{array}$ & $\begin{array}{l}08- \\
\text { Sep- } \\
05\end{array}$ & $\begin{array}{l}\text { Andrus Voitk } \\
\text { 05.09.08.av03 }\end{array}$ & DAOM744411 \\
\hline$\underset{f}{\mathrm{MH}} 473374$ & $\begin{array}{l}\text { CANADA, NL, Searston Provincial Park } \\
47.835030 N, 59.337496 W ; 2\end{array}$ & $\begin{array}{l}\text { heath } \\
\text { moss }\end{array}$ & $\begin{array}{l}05- \\
\text { Jun- } \\
10\end{array}$ & $\begin{array}{l}\text { Joe Brazil } \\
\text { 10.06.05.av07 }\end{array}$ & DAOM744410 \\
\hline $\begin{array}{l}\text { MH473373 } \\
f\end{array}$ & $\begin{array}{l}\text { CANADA, NL, Searston Provincial Park } \\
47.835030 \mathrm{~N}, 59.337496 \mathrm{~W} ; 2\end{array}$ & $\begin{array}{l}\text { heath } \\
\text { moss }\end{array}$ & $\begin{array}{l}05- \\
\text { Jun- } \\
10\end{array}$ & $\begin{array}{l}\text { Andrus Voitk } \\
\text { 10.06.05.av05 }\end{array}$ & DAOM744409 \\
\hline $\begin{array}{l}\text { MH473371 } \\
f\end{array}$ & $\begin{array}{l}\text { CANADA, NL, Gros Morne National } \\
\text { Park, trail to Wigwam Lake } \\
9.430075 \mathrm{~N}, 57.744673 \mathrm{~W} ; 68\end{array}$ & $\begin{array}{l}\text { fen } \\
\text { Sphagnum }\end{array}$ & $\begin{array}{l}01- \\
\text { Nov- } \\
05\end{array}$ & $\begin{array}{l}\text { Andrus Voitk } \\
\text { 05.11.01.av01 }\end{array}$ & DAOM744405 \\
\hline
\end{tabular}

a Omphalina pyxidata complex, unidentified species-code name given to the single species of Omphalina found in NL, mostly recorded as Omphalina cf. pyxidata.

${ }^{\mathrm{b}}$ Arranged in the order they appear in Fig. 1, followed by sequenced species not in Fig. 1.

c Sequence generated at Tartu University.

d Sequence generated at the Field Museum.

e Voucher collection lost in transit on return from sequencing.

f Sequence generated at Bridgwater College,

g Sequence generated by Osmundson et al. (2012)

h Sequence generated by Landry \& Bérubé, direct submission to GenBank, no associated publication. 


\begin{tabular}{|c|c|c|c|c|c|}
\hline $\begin{array}{l}\text { CODE: } \\
\text { GenBank } \\
\text { above, } \\
\text { UNITE } \\
\text { below }\end{array}$ & $\begin{array}{l}\text { COUNTRY, STATE/PROVINCE, Town, } \\
\text { locality above, } \\
\text { Coordinates (degree decimal); } \\
\text { elevation (m asl) below }\end{array}$ & $\begin{array}{l}\text { Habitat } \\
\text { above, } \\
\text { Substrate } \\
\text { below }\end{array}$ & $\begin{array}{l}\text { Date } \\
\text { DD- } \\
\text { Mon- } \\
\text { YY }\end{array}$ & $\begin{array}{l}\text { Collector } \\
\text { above } \\
\text { Collection } \\
\text { code below }\end{array}$ & $\begin{array}{l}\text { Herbarium } \\
\text { code: } \\
\text { Main above, } \\
\text { copy below }\end{array}$ \\
\hline $\begin{array}{l}\text { MN992480 } \\
\mathrm{g}\end{array}$ & CANADA, QC & & $\begin{array}{l}09- \\
\text { Sep- } \\
15\end{array}$ & $\begin{array}{l}\text { Renee Lebeuf } \\
\text { ANT255- } \\
\text { HRL2125 }\end{array}$ & \\
\hline $\begin{array}{l}\text { MN992479 } \\
\text { g }\end{array}$ & CANADA, QC & & $\begin{array}{l}09- \\
\text { Sep- } \\
15\end{array}$ & $\begin{array}{l}\text { Renee Lebeuf } \\
\text { ANT256- } \\
\text { HRL2124 }\end{array}$ & \\
\hline $\begin{array}{l}\text { UDB032201 } \\
\mathrm{c}\end{array}$ & $\begin{array}{l}\text { CANADA, NL, Labrador, L'Anse l'Amour } \\
\text { sand dunes } \\
51.476^{\circ} \mathrm{N}, 56.874^{\circ} \mathrm{W} ; 4 \mathrm{~m} \text { asl }\end{array}$ & $\begin{array}{l}\text { sand dune } \\
\text { moss }\end{array}$ & $\begin{array}{l}16- \\
\text { Aug- } \\
08\end{array}$ & $\begin{array}{l}\text { Esteri Ohenoja } \\
\text { LS-156 (ad3) }\end{array}$ & TUF117473 \\
\hline $\begin{array}{l}\text { UDB032202 } \\
\mathrm{c}\end{array}$ & $\begin{array}{l}\text { CANADA, NL, Sandy Cove (GNP) } \\
\text { 21U, NAD83, E523750, N5689462 }\end{array}$ & $\begin{array}{l}\text { limestone } \\
\text { barrens } \\
\text { peat }\end{array}$ & $\begin{array}{l}5- \\
\text { Oct- } \\
16\end{array}$ & $\begin{array}{l}\text { Michael } \\
\text { Burzynski } \\
\text { 16.10.05.av06 }\end{array}$ & TUF117475 \\
\hline $\begin{array}{l}\text { UDB032728 } \\
\text { c }\end{array}$ & $\begin{array}{l}\text { CANADA, NL, Labrador, outside } \\
\text { Forteau }\end{array}$ & $\begin{array}{l}\text { heath } \\
\text { moss }\end{array}$ & $\begin{array}{l}5- \\
\text { Sep- } \\
05\end{array}$ & $\begin{array}{l}\text { Tony Wright } \\
\text { GM5-443 }\end{array}$ & TUF117474 \\
\hline $\begin{array}{l}\text { UDB011424 } \\
\text { c }\end{array}$ & ESTONIA & $\begin{array}{l}\text { old firepit } \\
\text { site }\end{array}$ & $\begin{array}{l}31- \\
\text { Oct- } \\
07\end{array}$ & Vello Liiv & TUF106200 \\
\hline $\begin{array}{l}\text { MH473369 } \\
f\end{array}$ & $\begin{array}{l}\text { CANADA, NL, Ferolle Point } \\
51.020619 \mathrm{~N}, 57.094016 \mathrm{~W} ; 6\end{array}$ & $\begin{array}{l}\text { heath } \\
\text { Sphagnum } \\
\text { \& moss }\end{array}$ & $\begin{array}{l}29- \\
\text { Jun- } \\
05\end{array}$ & $\begin{array}{l}\text { Andrus Voitk } \\
\text { 05.06.29.av01 }\end{array}$ & DAOM744403 \\
\hline$\underset{f}{M H 473370}$ & $\begin{array}{l}\text { CANADA, NL, Great Brehat, Great } \\
\text { Brehat moor } 51.426534 \mathrm{~N}, \\
55.498613 \mathrm{~W} ; 14\end{array}$ & $\begin{array}{l}\text { heath } \\
\text { Sphagnum } \\
\& \text { moss }\end{array}$ & $\begin{array}{l}09- \\
\text { Jun- } \\
10\end{array}$ & $\begin{array}{l}\text { Andrus Voitk } \\
\text { 10.07.09.av02 }\end{array}$ & DAOM744404 \\
\hline
\end{tabular}

a Omphalina pyxidata complex, unidentified species-code name given to the single species of Omphalina found in NL, mostly recorded as Omphalina cf. pyxidata.

${ }^{\mathrm{b}}$ Arranged in the order they appear in Fig. 1, followed by sequenced species not in Fig. 1.

c Sequence generated at Tartu University.

d Sequence generated at the Field Museum.

e Voucher collection lost in transit on return from sequencing.

${ }^{\mathrm{f}}$ Sequence generated at Bridgwater College,

g Sequence generated by Osmundson et al. (2012)

h Sequence generated by Landry \& Bérubé, direct submission to GenBank, no associated publication. 


\begin{tabular}{|c|c|c|c|c|c|}
\hline $\begin{array}{l}\text { CODE: } \\
\text { GenBank } \\
\text { above, } \\
\text { UNITE } \\
\text { below }\end{array}$ & $\begin{array}{l}\text { COUNTRY, STATE/PROVINCE, Town, } \\
\text { locality above, } \\
\text { Coordinates (degree decimal); } \\
\text { elevation (m asl) below }\end{array}$ & $\begin{array}{l}\text { Habitat } \\
\text { above, } \\
\text { Substrate } \\
\text { below }\end{array}$ & $\begin{array}{l}\text { Date } \\
\text { DD- } \\
\text { Mon- } \\
\text { YY }\end{array}$ & $\begin{array}{l}\text { Collector } \\
\text { above } \\
\text { Collection } \\
\text { code below }\end{array}$ & $\begin{array}{l}\text { Herbarium } \\
\text { code: } \\
\text { Main above, } \\
\text { copy below }\end{array}$ \\
\hline $\begin{array}{l}\text { MH473372 } \\
f\end{array}$ & $\begin{array}{l}\text { CANADA, NL, Gros Morne National } \\
\text { Park, Western Brook Pond trail } \\
49.786176 N, 57.862653 W ; 24\end{array}$ & $\begin{array}{l}\text { bog } \\
\text { Sphagnum }\end{array}$ & $\begin{array}{l}09- \\
\text { Jun- } \\
07\end{array}$ & $\begin{array}{l}\text { Andrus Voitk } \\
\text { 07.09.06.av08 }\end{array}$ & DAOM744406 \\
\hline $\begin{array}{l}\text { MH473376 } \\
f\end{array}$ & $\begin{array}{l}\text { CANADA, QC, Saint-Augustin, Saint- } \\
\text { Augustin archipelago } 51.175867 \mathrm{~N}, \\
58.479824 \mathrm{~W} ; 2\end{array}$ & $\begin{array}{l}\text { bog } \\
\text { Sphagnum }\end{array}$ & $\begin{array}{l}24- \\
\text { Jul- } \\
07\end{array}$ & $\begin{array}{l}\text { Andrus Voitk } \\
\text { 07.07.24.av01 }\end{array}$ & DAOM744412 \\
\hline \multicolumn{6}{|c|}{$\begin{array}{l}\text { a Omphalina pyxidata complex, unidentified species-code name given to the single species of Omphalina found } \\
\text { in NL, mostly recorded as Omphalina cf. pyxidata. }\end{array}$} \\
\hline \multicolumn{6}{|c|}{ b Arranged in the order they appear in Fig. 1, followed by sequenced species not in Fig. 1.} \\
\hline \multicolumn{6}{|c|}{${ }^{\mathrm{c}}$ Sequence generated at Tartu University. } \\
\hline \multicolumn{6}{|c|}{ d Sequence generated at the Field Museum. } \\
\hline \multicolumn{6}{|c|}{ e Voucher collection lost in transit on return from sequencing. } \\
\hline \multicolumn{6}{|c|}{ f Sequence generated at Bridgwater College, } \\
\hline \multicolumn{6}{|c|}{ g Sequence generated by Osmundson et al. (2012) } \\
\hline
\end{tabular}


Table 3

Specimens of sphagnicolous arrhenias examined ${ }^{a}$

\begin{tabular}{|c|c|c|c|c|c|c|}
\hline $\begin{array}{l}\text { GenBank } \\
\text { number top } \\
\text { UNITE below }\end{array}$ & $\begin{array}{l}\text { Phylogenetic } \\
\text { ID b }\end{array}$ & $\begin{array}{l}\text { COUNTRY, } \\
\text { STATE/PROVINCE, } \\
\text { Town, locality } \\
\text { above Coordinates } \\
\text { (degree decimal); } \\
\text { elevation (m asl) } \\
\text { below }\end{array}$ & $\begin{array}{l}\text { Habitat } \\
\text { above, } \\
\text { Substrate } \\
\text { below }\end{array}$ & Date & $\begin{array}{l}\text { Collector } \\
\text { above } \\
\text { Collection } \\
\text { number below }\end{array}$ & $\begin{array}{l}\text { Herbarium } \\
\text { number: Main } \\
\text { above copy } \\
\text { below }\end{array}$ \\
\hline $\begin{array}{l}\text { OM422825 c } \\
\text { UDB038318 }\end{array}$ & $\begin{array}{l}\text { Arrhenia } \\
\text { bigelowii }\end{array}$ & $\begin{array}{l}\text { CANADA, NL, } \\
\text { Labrador, Battle } \\
\text { Harbour, Great } \\
\text { Caribou Island, } \\
\text { Indian Cove } \\
52.267245^{\circ} \mathrm{N}, \\
55.629755^{\circ} \mathrm{W} ; 42\end{array}$ & $\begin{array}{l}\text { bog } \\
\text { Sphagnum }\end{array}$ & $\begin{array}{l}\text { 21-Aug- } \\
18\end{array}$ & $\begin{array}{l}\text { Michael } \\
\text { Burzynski } \\
\text { TCMB16 }\end{array}$ & $\begin{array}{l}\text { DAOMXXXXXX } \\
\text { TUF117647 }\end{array}$ \\
\hline $\begin{array}{l}\text { OM422827 c } \\
\text { UDB038320 }\end{array}$ & $\begin{array}{l}\text { Arrhenia } \\
\text { bigelowii }\end{array}$ & $\begin{array}{l}\text { CANADA, NL, } \\
\text { Labrador, Battle } \\
\text { Harbour, Great } \\
\text { Caribou Island, } \\
52.273339^{\circ} \mathrm{N}, \\
55.591108^{\circ} \mathrm{W} ; 38\end{array}$ & $\begin{array}{l}\text { bog } \\
\text { Sphagnum }\end{array}$ & $\begin{array}{l}20 \text {-Aug- } \\
18\end{array}$ & $\begin{array}{l}\text { Michael } \\
\text { Burzynski } \\
\text { CEMB-01 }\end{array}$ & $\begin{array}{l}\text { DAOMXXXXXX } \\
\text { TUF117649 }\end{array}$ \\
\hline $\begin{array}{l}\text { OM422829 c } \\
\text { UDB038322 }\end{array}$ & $\begin{array}{l}\text { Arrhenia } \\
\text { bigelowii }\end{array}$ & $\begin{array}{l}\text { CANADA, NL, } \\
\text { Labrador, Battle } \\
\text { Harbour, Great } \\
\text { Caribou Island, } \\
\text { Indian Cove } \\
52.267245^{\circ} \mathrm{N}, \\
55.629755^{\circ} \mathrm{W} ; 42\end{array}$ & $\begin{array}{l}\text { bog } \\
\text { Sphagnum }\end{array}$ & $\begin{array}{l}21 \text {-Aug- } \\
18\end{array}$ & $\begin{array}{l}\text { Andrus Voitk } \\
\text { TCAV11 }\end{array}$ & $\begin{array}{l}\text { DAOMXXXXXX } \\
\text { TUF117651 }\end{array}$ \\
\hline $\begin{array}{l}\text { OM422830 c } \\
\text { UDB038323 }\end{array}$ & $\begin{array}{l}\text { Arrhenia } \\
\text { bigelowii }\end{array}$ & $\begin{array}{l}\text { CANADA, NL, } \\
\text { Labrador, Battle } \\
\text { Harbour, Great } \\
\text { Caribou Island, } \\
\text { Indian Cove } \\
52.267245^{\circ} \mathrm{N}, \\
55.629755^{\circ} \mathrm{W} ; 42\end{array}$ & $\begin{array}{l}\text { bog } \\
\text { Sphagnum }\end{array}$ & $\begin{array}{l}\text { 21-Aug- } \\
18\end{array}$ & $\begin{array}{l}\text { Maria Voitk } \\
\text { TCMV01 }\end{array}$ & $\begin{array}{l}\text { DAOMXXXXXX } \\
\text { TUF117652 }\end{array}$ \\
\hline $\mathrm{MH} 473353^{\mathrm{d}}$ & $\begin{array}{l}\text { Arrhenia } \\
\text { bigelowii }\end{array}$ & $\begin{array}{l}\text { CANADA, NL, near } \\
\text { Great Brehat, } \\
\text { Great Brehat moor } \\
51.426534 \mathrm{~N}, \\
55.498613 \mathrm{~W} ; 14\end{array}$ & $\begin{array}{l}\text { bog } \\
\text { Sphagnum }\end{array}$ & $\begin{array}{l}\text { 09-Jul- } \\
10\end{array}$ & $\begin{array}{l}\text { Andrus Voitk } \\
\text { 10.07.09.av04 }\end{array}$ & DAOM744389 \\
\hline $\mathrm{MH} 473349^{\mathrm{d}}$ & $\begin{array}{l}\text { Arrhenia } \\
\text { bigelowii }\end{array}$ & $\begin{array}{l}\text { CANADA, NL, } \\
\text { Trinity East, near } \\
\text { Port Rexton, } \\
\text { Skewink trail } \\
\text { 48.365623N, } \\
\text { 53.332913W; } 18\end{array}$ & $\begin{array}{l}\text { bog } \\
\text { Sphagnum }\end{array}$ & $\begin{array}{l}\text { 21-Jun- } \\
07\end{array}$ & $\begin{array}{l}\text { Andrus Voitk } \\
\text { 07.06.21.av02 }\end{array}$ & DAOM744393 \\
\hline MH473350 d & $\begin{array}{l}\text { Arrhenia } \\
\text { bigelowii }\end{array}$ & $\begin{array}{l}\text { CANADA, NL, near } \\
\text { Botwood, New Bay } \\
\text { Pond 49.099799N, } \\
55.581375 \mathrm{~W} ; 111\end{array}$ & $\begin{array}{l}\text { bog } \\
\text { Sphagnum }\end{array}$ & $\begin{array}{l}\text { 26-Jun- } \\
07\end{array}$ & $\begin{array}{l}\text { Andrus Voitk } \\
07.06 .26 \text {.av01 }\end{array}$ & DAOM744388 \\
\hline $\mathrm{MH} 473351^{\mathrm{d}}$ & $\begin{array}{l}\text { Arrhenia } \\
\text { bigelowii }\end{array}$ & $\begin{array}{l}\text { CANADA, QC, } \\
\text { Saint-Augustin, } \\
\text { Saint-Augustin } \\
\text { archipelago } \\
51.175867 \mathrm{~N}, \\
58.479824 \mathrm{~W} ; 2\end{array}$ & Sphagnum & $\begin{array}{l}\text { 23-Jul- } \\
07\end{array}$ & $\begin{array}{l}\text { Andrus Voitk } \\
\text { 07.07.23.av07 }\end{array}$ & DAOM744395 \\
\hline
\end{tabular}




\begin{tabular}{|c|c|c|c|c|c|c|}
\hline $\begin{array}{l}\text { GenBank } \\
\text { number top } \\
\text { UNITE below }\end{array}$ & $\begin{array}{l}\text { Phylogenetic } \\
\text { ID }^{\mathrm{b}}\end{array}$ & $\begin{array}{l}\text { COUNTRY, } \\
\text { STATE/PROVINCE, } \\
\text { Town, locality } \\
\text { above Coordinates } \\
\text { (degree decimal); } \\
\text { elevation (m asl) } \\
\text { below }\end{array}$ & $\begin{array}{l}\text { Habitat } \\
\text { above, } \\
\text { Substrate } \\
\text { below }\end{array}$ & Date & $\begin{array}{l}\text { Collector } \\
\text { above } \\
\text { Collection } \\
\text { number below }\end{array}$ & $\begin{array}{l}\text { Herbarium } \\
\text { number: Main } \\
\text { above copy } \\
\text { below }\end{array}$ \\
\hline $\mathrm{MH} 473352^{\mathrm{d}}$ & $\begin{array}{l}\text { Arrhenia } \\
\text { bigelowii }\end{array}$ & $\begin{array}{l}\text { CANADA, QC, } \\
\text { Saint-Augustin, } \\
\text { Saint-Augustin } \\
\text { archipelago } \\
51.175867 \mathrm{~N}, \\
\text { 58.479824W; } 2\end{array}$ & $\begin{array}{l}\text { bog } \\
\text { Sphagnum }\end{array}$ & $\begin{array}{l}\text { 23-Jul- } \\
07\end{array}$ & $\begin{array}{l}\text { Andrus Voitk } \\
\text { 07.07.23.av09 }\end{array}$ & DAOM744394 \\
\hline $\begin{array}{l}\text { MH473348d, } \\
e\end{array}$ & $\begin{array}{l}\text { Arrhenia } \\
\text { bigelowii } \\
\text { holotype }\end{array}$ & $\begin{array}{l}\text { CANADA, NL, } \\
\text { Rocky Harbour, } \\
\text { Rocky Harbour } \\
\text { bog 49.577694N, } \\
57.898916 \text { W; } 35\end{array}$ & $\begin{array}{l}\text { bog } \\
\text { Sphagnum }\end{array}$ & $\begin{array}{l}\text { 05-Jul- } \\
04\end{array}$ & $\begin{array}{l}\text { Andrus Voitk } \\
\text { 04.07.05.av03 }\end{array}$ & DAOM744391 \\
\hline $\mathrm{MH} 473347^{\mathrm{d}}$ & $\begin{array}{l}\text { Arrhenia } \\
\text { bigelowii }\end{array}$ & $\begin{array}{l}\text { CANADA, NL, } \\
\text { Labrador, near Red } \\
\text { Bay Tracy's Hill } \\
51.72972 \mathrm{~N}, \\
56.447783 \mathrm{~W} ; 138\end{array}$ & $\begin{array}{l}\text { bog } \\
\text { Sphagnum }\end{array}$ & $\begin{array}{l}\text { 15-Jun- } \\
05\end{array}$ & $\begin{array}{l}\text { Andrus Voitk } \\
\text { 05.06.15.av01 }\end{array}$ & $\mathrm{n} / \mathrm{a}^{\mathrm{e}}$ \\
\hline $\begin{array}{l}\text { MH198227 c } \\
\text { UDB034637 }\end{array}$ & $\begin{array}{l}\text { Arrhenia } \\
\text { bigelowii }\end{array}$ & $\begin{array}{l}\text { SWEDEN, } \\
\text { Sydbillingens } \\
\text { Nature Reserve, } \\
\text { Säxtingen }\end{array}$ & & $\begin{array}{l}\text { 19-Jun- } \\
86\end{array}$ & $\begin{array}{l}\text { Anders } \\
\text { Dahlberg } \\
86018\end{array}$ & GB-0072229 \\
\hline $\begin{array}{l}\text { MH198189 c } \\
\text { UDB024397 }\end{array}$ & $\begin{array}{l}\text { Arrhenia } \\
\text { bigelowii }\end{array}$ & $\begin{array}{l}\text { FINLAND, Pohjois- } \\
\text { Savo, } \\
\text { Vehmersalmi, } \\
\text { Rytökylä }\end{array}$ & & $\begin{array}{l}26-S e p- \\
93\end{array}$ & $\begin{array}{l}\text { Ilkka } \\
\text { Kytövuori 92- } \\
147\end{array}$ & H6057135 \\
\hline $\begin{array}{l}\text { MH198188 c } \\
\text { UDB024396 }\end{array}$ & $\begin{array}{l}\text { Arrhenia } \\
\text { bigelowii }\end{array}$ & $\begin{array}{l}\text { FINLAND, Pohjois- } \\
\text { Häme, Virrat, } \\
\text { Hauhuu }\end{array}$ & & $\begin{array}{l}\text { 05-Aug- } \\
90\end{array}$ & $\begin{array}{l}\text { llkka } \\
\text { Kytövuori 90- } \\
133\end{array}$ & H6057146 \\
\hline $\begin{array}{l}\text { MH198195 c } \\
\text { UDB024405 }\end{array}$ & $\begin{array}{l}\text { Arrhenia } \\
\text { bigelowii }\end{array}$ & $\begin{array}{l}\text { GERMANY, Bayern, } \\
\text { Garmisch- } \\
\text { Partenkirchen, } \\
\text { Saulgrub }\end{array}$ & & $\begin{array}{l}\text { 13-Jun- } \\
96\end{array}$ & $\begin{array}{l}\text { Christoph } \\
\text { Johannes } \\
\text { Hahn }\end{array}$ & M-0276634 \\
\hline $\begin{array}{l}\text { MH198192 c } \\
\text { UDB024401 }\end{array}$ & $\begin{array}{l}\text { Arrhenia } \\
\text { bigelowii }\end{array}$ & $\begin{array}{l}\text { SWEDEN, } \\
\text { Södermanland, } \\
\text { Vårdinge, } \\
\text { Sjuendaskogen, } \\
\text { between St. and L. } \\
\text { Alsjön }\end{array}$ & & $\begin{array}{l}\text { 09-Aug- } \\
94\end{array}$ & $\begin{array}{l}\text { Klas } \\
\text { Jaederfeldt }\end{array}$ & S-F277529 \\
\hline $\begin{array}{l}\text { MH198190 c } \\
\text { UDB024398 }\end{array}$ & $\begin{array}{l}\text { Arrhenia } \\
\text { bigelowii }\end{array}$ & $\begin{array}{l}\text { SWEDEN, Uppland } \\
\text { Hållnäs par., Lake } \\
\text { Fräkensjön (= } 4 \\
\text { km N of Hållnäs) }\end{array}$ & & $\begin{array}{l}06-A u g- \\
83\end{array}$ & $\begin{array}{l}\text { Nils } \\
\text { Lundquist } \\
14499\end{array}$ & S-F277532 \\
\hline $\begin{array}{l}\text { MH198219 c } \\
\text { UDB034625 }\end{array}$ & $\begin{array}{l}\text { Arrhenia } \\
\text { bigelowii }\end{array}$ & $\begin{array}{l}\text { CANADA, NL, Gros } \\
\text { Morne National } \\
\text { Park, bog at foot } \\
\text { of Gros Morne Mt. } \\
49.577694 \mathrm{~N}, \\
57.898916 \mathrm{~W} ; 35\end{array}$ & $\begin{array}{l}\text { bog } \\
\text { Sphagnum }\end{array}$ & $\begin{array}{l}\text { 12-Aug- } \\
08\end{array}$ & $\begin{array}{l}\text { Andrus Voitk } \\
\text { 08.08.12.av05 }\end{array}$ & $\begin{array}{l}\text { DAOM744390 } \\
\text { TUF117583 }\end{array}$ \\
\hline
\end{tabular}




\begin{tabular}{|c|c|c|c|c|c|c|}
\hline $\begin{array}{l}\text { GenBank } \\
\text { number top } \\
\text { UNITE below }\end{array}$ & $\begin{array}{l}\text { Phylogenetic } \\
\text { ID }^{\mathrm{b}}\end{array}$ & $\begin{array}{l}\text { COUNTRY, } \\
\text { STATE/PROVINCE, } \\
\text { Town, locality } \\
\text { above Coordinates } \\
\text { (degree decimal); } \\
\text { elevation (m asl) } \\
\text { below }\end{array}$ & $\begin{array}{l}\text { Habitat } \\
\text { above, } \\
\text { Substrate } \\
\text { below }\end{array}$ & Date & $\begin{array}{l}\text { Collector } \\
\text { above } \\
\text { Collection } \\
\text { number below }\end{array}$ & $\begin{array}{l}\text { Herbarium } \\
\text { number: Main } \\
\text { above copy } \\
\text { below }\end{array}$ \\
\hline $\begin{array}{l}\text { MH198220 c } \\
\text { UDB034626 }\end{array}$ & $\begin{array}{l}\text { Arrhenia } \\
\text { bigelowii }\end{array}$ & $\begin{array}{l}\text { CANADA, NL, } \\
\text { Labrador, Battle } \\
\text { Harbour, Great } \\
\text { Caribou Island, } \\
\text { Indian Cove } \\
52.2668 N, \\
55.648 W ; 17\end{array}$ & $\begin{array}{l}\text { bog } \\
\text { Sphagnum }\end{array}$ & $\begin{array}{l}\text { 19-Aug- } \\
08\end{array}$ & $\begin{array}{l}\text { Andrus Voitk } \\
\text { BH-018 (IC57) }\end{array}$ & TUF117584 \\
\hline $\begin{array}{l}\text { MH198204 c } \\
\text { UDB032052 }\end{array}$ & $\begin{array}{l}\text { Arrhenia } \\
\text { bigelowii }\end{array}$ & $\begin{array}{l}\text { GERMANY, Baden- } \\
\text { Württemberg, } \\
\text { Schwarzwald, } \\
\text { Breisgau- } \\
\text { Hochschwarzwald }\end{array}$ & & $\begin{array}{l}\text { 05-Jul- } \\
05\end{array}$ & Doris Laber & KR-0003833 \\
\hline $\begin{array}{l}\text { MH198205 c } \\
\text { UDB032053 }\end{array}$ & $\begin{array}{l}\text { Arrhenia } \\
\text { bigelowii }\end{array}$ & $\begin{array}{l}\text { GERMANY, Baden- } \\
\text { Württemberg, } \\
\text { Schwarzwald, } \\
\text { Breisgau- } \\
\text { Hochschwarzwald }\end{array}$ & & $\begin{array}{l}18-J u l- \\
84\end{array}$ & Doris Laber & KR-0004262 \\
\hline $\begin{array}{l}\text { MH198207 c } \\
\text { UDB032056 }\end{array}$ & $\begin{array}{l}\text { Arrhenia } \\
\text { bigelowii }\end{array}$ & $\begin{array}{l}\text { GERMANY, Baden- } \\
\text { Württemberg, } \\
\text { Schwarzwald, } \\
\text { Breisgau- } \\
\text { Hochschwarzwald }\end{array}$ & & $\begin{array}{l}\text { 19-Aug- } \\
06\end{array}$ & Doris Laber & KR-0004519 \\
\hline $\begin{array}{l}\text { MH198206 } \\
\text { UDB032054 }\end{array}$ & $\begin{array}{l}\text { Arrhenia } \\
\text { bigelowii }\end{array}$ & $\begin{array}{l}\text { GERMANY, Baden- } \\
\text { Württemberg, } \\
\text { Schwarzwald, } \\
\text { Breisgau- } \\
\text { Hochschwarzwald }\end{array}$ & & $\begin{array}{l}\text { 08-Sep- } \\
03\end{array}$ & Doris Laber & KR-0004576 \\
\hline $\begin{array}{l}\text { OM422833 c } \\
\text { UDB038353 }\end{array}$ & $\begin{array}{l}\text { Clitocybe } \\
\text { gerardiana } \\
\text { var. fusca } \\
\text { varietal } \\
\text { holotype }\end{array}$ & $\begin{array}{l}\text { USA, MICH, Luce } \\
\text { co., Pike Lake }\end{array}$ & $\begin{array}{l}\text { bog } \\
\text { Sphagnum }\end{array}$ & $\begin{array}{l}\text { 11-Sep- } \\
53\end{array}$ & $\begin{array}{l}\text { A. H. Smith } \\
42574\end{array}$ & $\begin{array}{l}\text { MICH10143 } \\
\text { TUF117871 }\end{array}$ \\
\hline $\begin{array}{l}\text { OM422824 c } \\
\text { UDB038317 }\end{array}$ & $\begin{array}{l}\text { Arrhenia } \\
\text { bigelowii }\end{array}$ & $\begin{array}{l}\text { CANADA, NL, } \\
\text { Labrador, Battle } \\
\text { Harbour, Great } \\
\text { Caribou Island, } \\
52.273339^{\circ} \mathrm{N}, \\
55.591108^{\circ} \mathrm{W} ; 38\end{array}$ & $\begin{array}{l}\text { bog } \\
\text { Sphagnum }\end{array}$ & $\begin{array}{l}20-A u g- \\
18\end{array}$ & $\begin{array}{l}\text { Michael } \\
\text { Burzynski } \\
\text { CEMB-02 }\end{array}$ & $\begin{array}{l}\text { DAOMXXXXXX } \\
\text { TUF117646 }\end{array}$ \\
\hline $\begin{array}{l}\text { OM422832 c } \\
\text { UDB038325 }\end{array}$ & $\begin{array}{l}\text { Arrhenia } \\
\text { bigelowii }\end{array}$ & $\begin{array}{l}\text { CANADA, NL, } \\
\text { Labrador, Battle } \\
\text { Harbour, Great } \\
\text { Caribou Island, } \\
\text { Indian Cove } \\
52.267245^{\circ} \mathrm{N}, \\
55.629755^{\circ} \mathrm{W} ; 42\end{array}$ & $\begin{array}{l}\text { barrens, } \\
\text { peat }\end{array}$ & $\begin{array}{l}\text { 21-Aug- } \\
18\end{array}$ & $\begin{array}{l}\text { Michael } \\
\text { Burzynski } \\
\text { TCMB09 }\end{array}$ & $\begin{array}{l}\text { DAOMXXXXXX } \\
\text { TUF117657 }\end{array}$ \\
\hline $\mathrm{MH} 473354^{\mathrm{d}}$ & $\begin{array}{l}\text { Arrhenia } \\
\text { bigelowii }\end{array}$ & $\begin{array}{l}\text { CANADA, NL, near } \\
\text { Stephenville } \\
48.542358 N, \\
58.118079 W ; 24\end{array}$ & $\begin{array}{l}\text { bog } \\
\text { Sphagnum }\end{array}$ & $\begin{array}{l}\text { 08-Aug- } \\
07\end{array}$ & $\begin{array}{l}\text { Andrus Voitk } \\
\text { 07.08.08.av02 }\end{array}$ & DAOM744392 \\
\hline
\end{tabular}




\begin{tabular}{|c|c|c|c|c|c|c|}
\hline $\begin{array}{l}\text { GenBank } \\
\text { number top } \\
\text { UNITE below }\end{array}$ & $\begin{array}{l}\text { Phylogenetic } \\
\text { ID } \mathrm{b}\end{array}$ & $\begin{array}{l}\text { COUNTRY, } \\
\text { STATE/PROVINCE, } \\
\text { Town, locality } \\
\text { above Coordinates } \\
\text { (degree decimal); } \\
\text { elevation (m asl) } \\
\text { below }\end{array}$ & $\begin{array}{l}\text { Habitat } \\
\text { above, } \\
\text { Substrate } \\
\text { below }\end{array}$ & Date & $\begin{array}{l}\text { Collector } \\
\text { above } \\
\text { Collection } \\
\text { number below }\end{array}$ & $\begin{array}{l}\text { Herbarium } \\
\text { number: Main } \\
\text { above copy } \\
\text { below }\end{array}$ \\
\hline $\begin{array}{l}\text { OM422835 c, } \\
\text { UDB0802694 }\end{array}$ & $\begin{array}{l}\text { Arrhenia } \\
\text { bigelowii }\end{array}$ & $\begin{array}{l}\text { CANADA, NL, } \\
\text { Stephenville } \\
\text { Crossing, } \\
\text { 48.516017N, } \\
\text { 58.433798W; } 16\end{array}$ & $\begin{array}{l}\text { bog } \\
\text { Sphagnum }\end{array}$ & $\begin{array}{l}\text { 17-Aug- } \\
10\end{array}$ & $\begin{array}{l}\text { Andrus Voitk } \\
\text { 10.08.17.av01 }\end{array}$ & TUF117886 \\
\hline $\begin{array}{l}\text { MH198199 c } \\
\text { UDB032042 }\end{array}$ & $\begin{array}{l}\text { Arrhenia } \\
\text { gerardiana }\end{array}$ & $\begin{array}{l}\text { GERMANY, } \\
\text { Fürstenfeldbruck }\end{array}$ & & $\begin{array}{l}24-J u n- \\
73\end{array}$ & $\begin{array}{l}\text { Andreas } \\
\text { Bresinsky }\end{array}$ & M-0276635 \\
\hline $\begin{array}{l}\text { MH198191 c } \\
\text { UDB024399 }\end{array}$ & $\begin{array}{l}\text { Arrhenia } \\
\text { gerardiana }\end{array}$ & $\begin{array}{l}\text { SWEDEN, Dalarna, } \\
\text { Orsa par., Tunturi, } \\
\text { NE of Mt. Stora } \\
\text { ( } 22 \mathrm{~km} \text { N of } \\
\text { Skattungbyn) }\end{array}$ & & $\begin{array}{l}10 \text {-Aug- } \\
93\end{array}$ & $\begin{array}{l}\text { Nils } \\
\text { Lundquist } \\
19520\end{array}$ & S-F277528 \\
\hline $\mathrm{MH} 473366^{\mathrm{d}}$ & $\begin{array}{l}\text { Arrhenia } \\
\text { gerardiana }\end{array}$ & $\begin{array}{l}\text { CANADA, NL, Gros } \\
\text { Morne National } \\
\text { Park, Western } \\
\text { Brook Pond trail } \\
49.786176 \mathrm{~N}, \\
57.862653 \mathrm{~W} ; 24\end{array}$ & $\begin{array}{l}\text { bog } \\
\text { Sphagnum }\end{array}$ & $\begin{array}{l}15 \text {-Jun- } \\
06\end{array}$ & $\begin{array}{l}\text { Andrus Voitk } \\
\text { 06.06.15.av04 }\end{array}$ & DAOM744373 \\
\hline $\begin{array}{l}\text { MH473357 d } \\
\text { MH473358 d }\end{array}$ & $\begin{array}{l}\text { Arrhenia } \\
\text { gerardiana }\end{array}$ & $\begin{array}{l}\text { CANADA, NL, } \\
\text { Cheesman Park } \\
47.632104 \mathrm{~N}, \\
59.256736 \mathrm{~W} ; 3\end{array}$ & $\begin{array}{l}\text { bog } \\
\text { Sphagnum }\end{array}$ & $\begin{array}{l}\text { 15-Jul- } \\
04\end{array}$ & $\begin{array}{l}\text { Andrus Voitk } \\
\text { 04.07.15.av01 }\end{array}$ & DAOM744371 \\
\hline MH473359 d & $\begin{array}{l}\text { Arrhenia } \\
\text { gerardiana }\end{array}$ & $\begin{array}{l}\text { CANADA, NL, } \\
\text { Rocky Harbour, } \\
\text { Rocky Harbour } \\
\text { bog 49.577694N, } \\
57.898916 \mathrm{~W} ; 35\end{array}$ & $\begin{array}{l}\text { bog } \\
\text { Sphagnum }\end{array}$ & $\begin{array}{l}\text { 03-Jul- } \\
05\end{array}$ & $\begin{array}{l}\text { Andrus Voitk } \\
\text { 05.07.03.av01 }\end{array}$ & DAOM744376 \\
\hline MH473355 d & $\begin{array}{l}\text { Arrhenia } \\
\text { gerardiana }\end{array}$ & $\begin{array}{l}\text { CANADA, NL, } \\
\text { Rocky Harbour, } \\
\text { Rocky Harbour } \\
\text { bog 49.577694N, } \\
57.898916 \mathrm{~W} ; 35\end{array}$ & $\begin{array}{l}\text { bog } \\
\text { Sphagnum }\end{array}$ & $\begin{array}{l}\text { 03-Jul- } \\
05\end{array}$ & $\begin{array}{l}\text { Andrus Voitk } \\
\text { 05.07.03.av02 }\end{array}$ & DAOM744377 \\
\hline $\mathrm{MH} 473367^{\mathrm{d}}$ & $\begin{array}{l}\text { Arrhenia } \\
\text { gerardiana }\end{array}$ & $\begin{array}{l}\text { CANADA, NL, } \\
\text { Rocky Harbour, } \\
\text { Rocky Harbour } \\
\text { bog 49.577694N, } \\
57.898916 \text { W; } 35\end{array}$ & $\begin{array}{l}\text { bog } \\
\text { Sphagnum }\end{array}$ & $\begin{array}{l}\text { 03-Jul- } \\
05\end{array}$ & $\begin{array}{l}\text { Andrus Voitk } \\
\text { 05.07.03.av03 }\end{array}$ & DAOM744375 \\
\hline $\mathrm{MH} 473365^{\mathrm{d}}$ & $\begin{array}{l}\text { Arrhenia } \\
\text { gerardiana }\end{array}$ & $\begin{array}{l}\text { CANADA, NL, near } \\
\text { Gillams, Gillams } \\
\text { bog } 49.011140 \mathrm{~N}, \\
58.066273 \mathrm{~W} ; 103\end{array}$ & $\begin{array}{l}\text { bog } \\
\text { Sphagnum }\end{array}$ & $\begin{array}{l}\text { 16-Jul- } \\
08\end{array}$ & $\begin{array}{l}\text { Andrus Voitk } \\
\text { 08.07.16.av01 }\end{array}$ & DAOM744372 \\
\hline $\mathrm{MH} 473361^{\mathrm{d}}$ & $\begin{array}{l}\text { Arrhenia } \\
\text { gerardiana }\end{array}$ & $\begin{array}{l}\text { CANADA, QC, } \\
\text { Saint-Augustin, } \\
\text { Saint-Augustin } \\
\text { archipelago } \\
51.175867 \mathrm{~N}, \\
58.479824 \mathrm{~W} ; 2\end{array}$ & $\begin{array}{l}\text { bog } \\
\text { Sphagnum }\end{array}$ & $\begin{array}{l}\text { 24-Jul- } \\
07\end{array}$ & $\begin{array}{l}\text { Andrus Voitk } \\
\text { 07.07.24.av02 }\end{array}$ & DAOM744387 \\
\hline
\end{tabular}




\begin{tabular}{|c|c|c|c|c|c|c|}
\hline $\begin{array}{l}\text { GenBank } \\
\text { number top } \\
\text { UNITE below }\end{array}$ & $\begin{array}{l}\text { Phylogenetic } \\
\text { ID b }\end{array}$ & $\begin{array}{l}\text { COUNTRY, } \\
\text { STATE/PROVINCE, } \\
\text { Town, locality } \\
\text { above Coordinates } \\
\text { (degree decimal); } \\
\text { elevation (m asl) } \\
\text { below }\end{array}$ & $\begin{array}{l}\text { Habitat } \\
\text { above, } \\
\text { Substrate } \\
\text { below }\end{array}$ & Date & $\begin{array}{l}\text { Collector } \\
\text { above } \\
\text { Collection } \\
\text { number below }\end{array}$ & $\begin{array}{l}\text { Herbarium } \\
\text { number: Main } \\
\text { above copy } \\
\text { below }\end{array}$ \\
\hline $\mathrm{MH} 47335^{\mathrm{d}}$ & $\begin{array}{l}\text { Arrhenia } \\
\text { gerardiana }\end{array}$ & $\begin{array}{l}\text { CANADA, QC, } \\
\text { Saint-Augustin, } \\
\text { Saint-Augustin } \\
\text { archipelago } \\
51.175867 \mathrm{~N}, \\
\text { 58.479824W; } 2\end{array}$ & $\begin{array}{l}\text { bog } \\
\text { Sphagnum }\end{array}$ & $\begin{array}{l}\text { 23-Jul- } \\
07\end{array}$ & $\begin{array}{l}\text { Andrus Voitk } \\
\text { 07.07.23.av02 }\end{array}$ & DAOM744386 \\
\hline MH473363 d & $\begin{array}{l}\text { Arrhenia } \\
\text { gerardiana }\end{array}$ & $\begin{array}{l}\text { CANADA, QC, } \\
\text { Saint-Augustin, } \\
\text { Saint-Augustin } \\
\text { archipelago } \\
51.175867 N, \\
58.479824 W ; 2\end{array}$ & $\begin{array}{l}\text { bog } \\
\text { Sphagnum }\end{array}$ & $\begin{array}{l}21-J u l- \\
07\end{array}$ & $\begin{array}{l}\text { Andrus Voitk } \\
\text { 07.07.21.av01 }\end{array}$ & DAOM744384 \\
\hline $\mathrm{MH} 473360^{\mathrm{d}}$ & $\begin{array}{l}\text { Arrhenia } \\
\text { gerardiana }\end{array}$ & $\begin{array}{l}\text { CANADA, NL, } \\
\text { Rocky Harbour, } \\
\text { Rocky Harbour } \\
\text { bog 49.577694N, } \\
57.898916 \text {; } 35\end{array}$ & $\begin{array}{l}\text { bog } \\
\text { Sphagnum }\end{array}$ & $\begin{array}{l}15 \text {-Jun- } \\
06\end{array}$ & $\begin{array}{l}\text { Andrus Voitk } \\
\text { 06.06.15.av02 }\end{array}$ & DAOM744378 \\
\hline $\mathrm{MH} 473362^{\mathrm{d}}$ & $\begin{array}{l}\text { Arrhenia } \\
\text { gerardiana }\end{array}$ & $\begin{array}{l}\text { CANADA, QC, } \\
\text { Saint-Augustin, } \\
\text { Saint-Augustin } \\
\text { archipelago } \\
51.175867 \mathrm{~N}, \\
\text { 58.479824W; } 2\end{array}$ & $\begin{array}{l}\text { bog } \\
\text { Sphagnum }\end{array}$ & $\begin{array}{l}\text { 23-Jul- } \\
07\end{array}$ & $\begin{array}{l}\text { Andrus Voitk } \\
\text { 07.07.23.av01 }\end{array}$ & DAOM744385 \\
\hline $\begin{array}{l}\text { MH198183 c } \\
\text { UDB024327 }\end{array}$ & $\begin{array}{l}\text { Arrhenia } \\
\text { gerardiana } \\
\text { epitype }\end{array}$ & $\begin{array}{l}\text { USA, New York, } \\
\text { Essex Co., North } \\
\text { Elba, Mount Marcy }\end{array}$ & $\begin{array}{l}\text { bog } \\
\text { Sphagnum }\end{array}$ & undated & Charles Peck & NYSd4725.1 \\
\hline $\begin{array}{l}\text { MH198221 } \\
\text { UDB034627 }\end{array}$ & $\begin{array}{l}\text { Arrhenia } \\
\text { gerardiana }\end{array}$ & $\begin{array}{l}\text { CANADA, NL, } \\
\text { Labrador, Battle } \\
\text { Harbour, Great } \\
\text { Caribou Island, } \\
\text { Indian Cove } \\
52.2668 \text {, } \\
55.648 W ; 17\end{array}$ & $\begin{array}{l}\text { bog } \\
\text { Sphagnum }\end{array}$ & $\begin{array}{l}\text { 19-Aug- } \\
08\end{array}$ & $\begin{array}{l}\text { Maria Voitk } \\
\text { BH-020 (IC54) }\end{array}$ & $\begin{array}{l}\text { DAOM744382 } \\
\text { TUF117585 }\end{array}$ \\
\hline $\begin{array}{l}\text { MH198222 c } \\
\text { UDB034628 }\end{array}$ & $\begin{array}{l}\text { Arrhenia } \\
\text { gerardiana }\end{array}$ & $\begin{array}{l}\text { CANADA, NL, } \\
\text { Labrador, Battle } \\
\text { Harbour, Great } \\
\text { Caribou Island, } \\
\text { Indian Cove } \\
52.2668 \text {, } \\
55.648 W ; 17\end{array}$ & $\begin{array}{l}\text { bog } \\
\text { Sphagnum }\end{array}$ & $\begin{array}{l}\text { 19-Aug- } \\
08\end{array}$ & $\begin{array}{l}\text { Anne } \\
\text { Marceau BH- } \\
022 \text { (IC55) }\end{array}$ & TUF117586 \\
\hline $\begin{array}{l}\text { MH198223 c } \\
\text { UDB034629 }\end{array}$ & $\begin{array}{l}\text { Arrhenia } \\
\text { gerardiana }\end{array}$ & $\begin{array}{l}\text { CANADA, NL, } \\
\text { Labrador, Battle } \\
\text { Harbour, Great } \\
\text { Caribou Island, } \\
\text { Indian Cove } \\
52.2668 \text {, } \\
55.648 W ; 17\end{array}$ & $\begin{array}{l}\text { bog } \\
\text { Sphagnum }\end{array}$ & $\begin{array}{l}\text { 19-Aug- } \\
08\end{array}$ & $\begin{array}{l}\text { Maria Voitk } \\
\text { BH-021 (IC56) }\end{array}$ & TUF117587 \\
\hline
\end{tabular}




\begin{tabular}{|c|c|c|c|c|c|c|}
\hline $\begin{array}{l}\text { GenBank } \\
\text { number top } \\
\text { UNITE below }\end{array}$ & $\begin{array}{l}\text { Phylogenetic } \\
\text { ID }^{\mathrm{b}}\end{array}$ & $\begin{array}{l}\text { COUNTRY, } \\
\text { STATE/PROVINCE, } \\
\text { Town, locality } \\
\text { above Coordinates } \\
\text { (degree decimal); } \\
\text { elevation (m asl) } \\
\text { below }\end{array}$ & $\begin{array}{l}\text { Habitat } \\
\text { above, } \\
\text { Substrate } \\
\text { below }\end{array}$ & Date & $\begin{array}{l}\text { Collector } \\
\text { above } \\
\text { Collection } \\
\text { number below }\end{array}$ & $\begin{array}{l}\text { Herbarium } \\
\text { number: Main } \\
\text { above copy } \\
\text { below }\end{array}$ \\
\hline $\begin{array}{l}\text { MH198224 c } \\
\text { UDB034631 }\end{array}$ & $\begin{array}{l}\text { Arrhenia } \\
\text { gerardiana }\end{array}$ & $\begin{array}{l}\text { CANADA, NL, } \\
\text { Rocky Harbour, } \\
\text { Rocky Harbour } \\
\text { bog } 49.577694 \mathrm{~N}, \\
57.898916 \mathrm{~W} ; 35\end{array}$ & $\begin{array}{l}\text { bog } \\
\text { Sphagnum }\end{array}$ & $\begin{array}{l}\text { 04-Jul- } \\
17\end{array}$ & $\begin{array}{l}\text { Michael } \\
\text { Burzynski } \\
\text { 17.07.04.av02 }\end{array}$ & $\begin{array}{l}\text { DAOM744379 } \\
\text { TUF117590 }\end{array}$ \\
\hline $\begin{array}{l}\text { MH198225 c } \\
\text { UDB034632 }\end{array}$ & $\begin{array}{l}\text { Arrhenia } \\
\text { gerardiana }\end{array}$ & $\begin{array}{l}\text { CANADA, NL, } \\
\text { Rocky Harbour, } \\
\text { Rocky Harbour } \\
\text { bog 49.577694N, } \\
57.898916 \text {; } 35\end{array}$ & $\begin{array}{l}\text { bog } \\
\text { Sphagnum }\end{array}$ & $\begin{array}{l}05-J u l- \\
17\end{array}$ & $\begin{array}{l}\text { Michael } \\
\text { Burzynski } \\
\text { 17.07.05.av04 }\end{array}$ & $\begin{array}{l}\text { DAOM744380 } \\
\text { TUF117591 }\end{array}$ \\
\hline $\begin{array}{l}\text { MH198226 } \\
\text { UDB034633 }\end{array}$ & $\begin{array}{l}\text { Arrhenia } \\
\text { gerardiana }\end{array}$ & $\begin{array}{l}\text { CANADA, NL, } \\
\text { Rocky Harbour, } \\
\text { Rocky Harbour } \\
\text { bog 49.577694N, } \\
57.898916 \text {; } 35\end{array}$ & $\begin{array}{l}\text { bog } \\
\text { Sphagnum }\end{array}$ & $\begin{array}{l}05-J u l- \\
17\end{array}$ & $\begin{array}{l}\text { Michael } \\
\text { Burzynski } \\
\text { 17.07.05.av05 }\end{array}$ & $\begin{array}{l}\text { DAOM744381 } \\
\text { TUF117592 }\end{array}$ \\
\hline $\begin{array}{l}\text { OM422826 } \\
\text { UDB038319 }\end{array}$ & $\begin{array}{l}\text { Arrhenia } \\
\text { gerardiana }\end{array}$ & $\begin{array}{l}\text { CANADA, NL, } \\
\text { Labrador, Battle } \\
\text { Harbour, Great } \\
\text { Caribou Island, } \\
52.273339^{\circ} \mathrm{N}, \\
55.591108^{\circ} \mathrm{W} ; 38\end{array}$ & $\begin{array}{l}\text { bog } \\
\text { Sphagnum }\end{array}$ & $\begin{array}{l}20 \text {-Aug- } \\
18\end{array}$ & $\begin{array}{l}\text { Andrus Voitk } \\
\text { CEAV-03 }\end{array}$ & $\begin{array}{l}\text { DAOMXXXXXX } \\
\text { TUF117648 }\end{array}$ \\
\hline $\begin{array}{l}\text { OM422831 c } \\
\text { UDB038324 }\end{array}$ & $\begin{array}{l}\text { Arrhenia } \\
\text { gerardiana }\end{array}$ & $\begin{array}{l}\text { CANADA, NL, } \\
\text { Labrador, Battle } \\
\text { Harbour, Great } \\
\text { Caribou Island, } \\
\text { Indian Cove } \\
52.267245^{\circ} \mathrm{N}, \\
55.629755^{\circ} \mathrm{W} ; 42\end{array}$ & $\begin{array}{l}\text { bog } \\
\text { Sphagnum }\end{array}$ & $\begin{array}{l}21 \text {-Aug- } \\
18\end{array}$ & $\begin{array}{l}\text { Michael } \\
\text { Burzynski } \\
\text { TCMB-04 }\end{array}$ & $\begin{array}{l}\text { DAOMXXXXXX } \\
\text { TUF117653 }\end{array}$ \\
\hline $\mathrm{MH} 473364^{\mathrm{d}}$ & $\begin{array}{l}\text { Arrhenia } \\
\text { gerardiana }\end{array}$ & $\begin{array}{l}\text { CANADA, NL, Gros } \\
\text { Morne National } \\
\text { Park, Western } \\
\text { Brook Pond trail } \\
49.786176 \mathrm{~N}, \\
57.862653 \mathrm{~W} ; 24\end{array}$ & $\begin{array}{l}\text { bog } \\
\text { Sphagnum }\end{array}$ & $\begin{array}{l}31-A u g- \\
08\end{array}$ & $\begin{array}{l}\text { Andrus Voitk } \\
\text { 08.08.31.av02 }\end{array}$ & DAOM744374 \\
\hline $\begin{array}{l}\text { MH198218 c } \\
\text { UDB034624 }\end{array}$ & $\begin{array}{l}\text { Arrhenia } \\
\text { gerardiana }\end{array}$ & $\begin{array}{l}\text { CANADA, } \\
\text { Labrador, W of } \\
\text { Konrad Brook } \\
\text { Pond 56.139413N, } \\
62.730548 W ; 50\end{array}$ & $\begin{array}{l}\text { bog } \\
\text { Sphagnum }\end{array}$ & $\begin{array}{l}\text { 28-Jul- } \\
08\end{array}$ & $\begin{array}{l}\text { Esteri } \\
\text { Ohenoja KL- } \\
014 \text { (K42) }\end{array}$ & $\begin{array}{l}\text { DAOM744383 } \\
\text { TUF117581 }\end{array}$ \\
\hline $\begin{array}{l}\text { OM422828 c } \\
\text { UDB038321 }\end{array}$ & $\begin{array}{l}\text { Arrhenia } \\
\text { gerardiana }\end{array}$ & $\begin{array}{l}\text { CANADA, NL, } \\
\text { Labrador, Battle } \\
\text { Harbour, Great } \\
\text { Caribou Island, } \\
52.273339^{\circ} \mathrm{N}, \\
55.591108^{\circ} \mathrm{W} ; 38\end{array}$ & $\begin{array}{l}\text { bog } \\
\text { Sphagnum }\end{array}$ & $\begin{array}{l}20 \text {-Aug- } \\
18\end{array}$ & $\begin{array}{l}\text { Maria Voitk } \\
\text { CEMV-02 }\end{array}$ & $\begin{array}{l}\text { DAOMXXXXXX } \\
\text { TUF117650 }\end{array}$ \\
\hline $\begin{array}{l}\text { OM422836 c, } \\
\text { g } \\
\text { UDB0802695 }\end{array}$ & $\begin{array}{l}\text { Arrhenia } \\
\text { gerardiana }\end{array}$ & $\begin{array}{l}\text { CANADA, NL, near } \\
\text { Stephenville }\end{array}$ & $\begin{array}{l}\text { bog } \\
\text { Sphagnum }\end{array}$ & $\begin{array}{l}\text { 13-Jul- } \\
10\end{array}$ & $\begin{array}{l}\text { Andrus Voitk } \\
\text { 10.07.13.av06 }\end{array}$ & TUF117887 \\
\hline
\end{tabular}




\begin{tabular}{|c|c|c|c|c|c|c|}
\hline $\begin{array}{l}\text { GenBank } \\
\text { number top } \\
\text { UNITE below }\end{array}$ & $\begin{array}{l}\text { Phylogenetic } \\
\text { ID b }\end{array}$ & $\begin{array}{l}\text { COUNTRY, } \\
\text { STATE/PROVINCE, } \\
\text { Town, locality } \\
\text { above Coordinates } \\
\text { (degree decimal); } \\
\text { elevation (m asl) } \\
\text { below }\end{array}$ & $\begin{array}{l}\text { Habitat } \\
\text { above, } \\
\text { Substrate } \\
\text { below }\end{array}$ & Date & $\begin{array}{l}\text { Collector } \\
\text { above } \\
\text { Collection } \\
\text { number below }\end{array}$ & $\begin{array}{l}\text { Herbarium } \\
\text { number: Main } \\
\text { above copy } \\
\text { below }\end{array}$ \\
\hline \multirow[t]{2}{*}{$n / a^{g, h}$} & \multirow{2}{*}{$\begin{array}{l}\text { Agaricus } \\
\text { gerardianus } \\
\text { holotype }\end{array}$} & \multirow[t]{2}{*}{ USA, New York } & bog & \multirow[t]{2}{*}{ undated } & \multirow[t]{2}{*}{ Charles Peck } & \multirow[t]{2}{*}{ NYSf4339.1-4 } \\
\hline & & & Sphagnum & & & \\
\hline \multirow{3}{*}{$\begin{array}{l}\text { MH198198 c } \\
\text { UDB32176 }\end{array}$} & \multirow{3}{*}{$\begin{array}{l}\text { Arrhenia } \\
\text { philonotis }\end{array}$} & \multirow{3}{*}{$\begin{array}{l}\text { CANADA, NL, } \\
\text { Labrador, L'Anse } \\
\text { Amour } \\
51.470074 N \text {, } \\
56.870960 W ; 2\end{array}$} & heath & \multirow{3}{*}{$\begin{array}{l}16-A u g- \\
08\end{array}$} & \multirow{3}{*}{$\begin{array}{l}\text { Esteri } \\
\text { Ohenoja LS- } \\
008 \\
(A D-14)\end{array}$} & \multirow[t]{3}{*}{ TUF117360 } \\
\hline & & & moss & & & \\
\hline & & & & & & \\
\hline $\begin{array}{l}\text { MH198187 } \\
\text { UDB024395 }\end{array}$ & $\begin{array}{l}\text { Arrhenia } \\
\text { philonotis }\end{array}$ & $\begin{array}{l}\text { FINLAND, } \\
\text { Uusimaa, } \\
\text { Nurmijärvi, } \\
\text { Klaukkala }\end{array}$ & & $\begin{array}{l}\text { 08-Jul- } \\
84\end{array}$ & $\begin{array}{l}\text { Pirkko Askola, } \\
\text { Tauno } \\
\text { Toivonen } \\
1413\end{array}$ & H6057134 \\
\hline $\begin{array}{l}\text { MH198203 c } \\
\text { UDB032501 }\end{array}$ & $\begin{array}{l}\text { Arrhenia } \\
\text { philonotis } \\
\text { epitype }\end{array}$ & $\begin{array}{l}\text { GERMANY, Baden- } \\
\text { Württemberg, } \\
\text { Schwarzwald, } \\
\text { Breisgau- } \\
\text { Hochschwarzwald }\end{array}$ & & $\begin{array}{l}29-J u l- \\
84\end{array}$ & Doris Laber & KR-0003880 \\
\hline $\begin{array}{l}\text { MH198202 }{ }^{\mathrm{C}} \\
\text { UDB032050 }\end{array}$ & $\begin{array}{l}\text { Arrhenia } \\
\text { philonotis }\end{array}$ & $\begin{array}{l}\text { GERMANY, Baden- } \\
\text { Württemberg, } \\
\text { Schwarzwald, } \\
\text { Breisgau- } \\
\text { Hochschwarzwald }\end{array}$ & & $\begin{array}{l}\text { 18-Aug- } \\
90\end{array}$ & Doris Laber & KR-0004132 \\
\hline $\begin{array}{l}\text { MH198194 }{ }^{\mathrm{C}} \\
\text { UDB024404 }\end{array}$ & $\begin{array}{l}\text { Arrhenia } \\
\text { philonotis }\end{array}$ & $\begin{array}{l}\text { GERMANY, } \\
\text { Oberbayern, Moor } \\
\text { am Eßsee }\end{array}$ & & $\begin{array}{l}28-J u n- \\
58\end{array}$ & J. Poelt & $M-0276633$ \\
\hline MH491520 i & $\begin{array}{l}\text { Arrhenia } \\
\text { philonotis }\end{array}$ & $\begin{array}{l}\text { CANADA, NL, } \\
\text { Labrador, Konrad }\end{array}$ & $\begin{array}{l}\text { bog } \\
\text { Sphagnum }\end{array}$ & $\begin{array}{l}30-J u l- \\
08\end{array}$ & $\begin{array}{l}\text { Michael } \\
\text { Burzynski }\end{array}$ & DAOM744400 \\
\hline & & $\begin{array}{l}56.278381 \mathrm{~N}, \\
62.933697 \mathrm{~W} ; 517\end{array}$ & & & KL-013 (T13) & \\
\hline MH491521 i & $\begin{array}{l}\text { Arrhenia } \\
\text { philonotis }\end{array}$ & $\begin{array}{l}\text { CANADA, NL, } \\
\text { Labrador, L'Anse } \\
\text { Amour } \\
51.470074 N \text {, } \\
56.870960 W ; 2\end{array}$ & $\begin{array}{l}\text { bog } \\
\text { Sphagnum }\end{array}$ & $\begin{array}{l}\text { 15-Aug- } \\
08\end{array}$ & $\begin{array}{l}\text { Maria Voitk } \\
\text { LS-010 } \\
\text { (LAT4) }\end{array}$ & $n / a^{j}$ \\
\hline $\begin{array}{l}\text { MH198197 c } \\
\text { UDB032175 }\end{array}$ & $\begin{array}{l}\text { Arrhenia } \\
\text { philonotis }\end{array}$ & $\begin{array}{l}\text { CANADA, NL, } \\
\text { Labrador, Konrad } \\
\text { Brook } \\
56.278381 \mathrm{~N}, \\
62.933697 \mathrm{~W} ; 517\end{array}$ & $\begin{array}{l}\text { bog } \\
\text { Sphagnum } \\
\text { \& moss }\end{array}$ & $\begin{array}{l}30-A u g- \\
08\end{array}$ & $\begin{array}{l}\text { Andrus Voitk } \\
\text { KL-015 (T3) }\end{array}$ & $\begin{array}{l}\text { DAOM744401 } \\
\text { TUF117359 }\end{array}$ \\
\hline U66449 k & $\begin{array}{l}\text { Arrhenia } \\
\text { philonotis }\end{array}$ & GREENLAND & & & $\begin{array}{l}\text { François } \\
\text { Lutzoni } \\
(1997)\end{array}$ & \\
\hline$M H 473368^{d}$ & $\begin{array}{l}\text { Arrhenia } \\
\text { philonotis }\end{array}$ & $\begin{array}{l}\text { NORWAY, near } \\
\text { Troms } \emptyset \text {, Kaldfjord } \\
69.679009 \mathrm{~N}, \\
18.737086 \mathrm{E} ; 75\end{array}$ & $\begin{array}{l}\text { fen, } \\
\text { moss and } \\
\text { Sphagnum }\end{array}$ & $\begin{array}{l}\text { 06-Jul- } \\
08\end{array}$ & $\begin{array}{l}\text { Andrus Voitk } \\
\text { 08.07.06.av01 }\end{array}$ & DAOM744402 \\
\hline
\end{tabular}




\begin{tabular}{|c|c|c|c|c|c|c|}
\hline $\begin{array}{l}\text { GenBank } \\
\text { number top } \\
\text { UNITE below }\end{array}$ & $\begin{array}{l}\text { Phylogenetic } \\
\text { ID } \mathrm{b}\end{array}$ & $\begin{array}{l}\text { COUNTRY, } \\
\text { STATE/PROVINCE, } \\
\text { Town, locality } \\
\text { above Coordinates } \\
\text { (degree decimal); } \\
\text { elevation (m asl) } \\
\text { below }\end{array}$ & $\begin{array}{l}\text { Habitat } \\
\text { above, } \\
\text { Substrate } \\
\text { below }\end{array}$ & Date & $\begin{array}{l}\text { Collector } \\
\text { above } \\
\text { Collection } \\
\text { number below }\end{array}$ & $\begin{array}{l}\text { Herbarium } \\
\text { number: Main } \\
\text { above copy } \\
\text { below }\end{array}$ \\
\hline $\begin{array}{l}\text { OM422834 } \\
\text { UDB039743 }\end{array}$ & $\begin{array}{l}\text { Agaricus } \\
\text { telmatiaeus } \\
\text { holotype }\end{array}$ & $\begin{array}{l}\text { UK, ENGLAND, } \\
\text { Yorkshire County, } \\
\text { Scarboro }\end{array}$ & Sphagnum & $\begin{array}{l}\text { 08-Nov- } \\
1882\end{array}$ & G.E. Massee & NY12555 \\
\hline $\begin{array}{l}\text { MH198211 c } \\
\text { UDB032770 }\end{array}$ & $\begin{array}{l}\text { Arrhenia } \\
\text { telmatiaea }\end{array}$ & $\begin{array}{l}\text { GERMANY, Baden- } \\
\text { Württemberg, } \\
\text { Schwarzwald, } \\
\text { Breisgau- } \\
\text { Hochschwarzwald }\end{array}$ & & $\begin{array}{l}05-0 c t- \\
86\end{array}$ & Doris Laber & KR-0004131 \\
\hline $\begin{array}{l}\text { MH198201 c } \\
\text { UDB032049 }\end{array}$ & $\begin{array}{l}\text { Arrhenia } \\
\text { telmatiaea }\end{array}$ & $\begin{array}{l}\text { GERMANY, Baden- } \\
\text { Württemberg, } \\
\text { Schwarzwald, } \\
\text { Breisgau- } \\
\text { Hochschwarzwald }\end{array}$ & & $\begin{array}{l}03-0 c t- \\
04\end{array}$ & Doris Laber & KR-0004167 \\
\hline $\begin{array}{l}\text { MH198200 C } \\
\text { UDB032048 }\end{array}$ & $\begin{array}{l}\text { Arrhenia } \\
\text { telmatiaea }\end{array}$ & $\begin{array}{l}\text { GERMANY, Baden- } \\
\text { Württemberg, } \\
\text { Schwarzwald, } \\
\text { Breisgau- } \\
\text { Hochschwarzwald }\end{array}$ & & $\begin{array}{l}10-0 c t- \\
07\end{array}$ & Doris Laber & KR-0004666 \\
\hline $\begin{array}{l}\text { MH198196 c } \\
\text { UDB032174 }\end{array}$ & $\begin{array}{l}\text { Arrhenia } \\
\text { telmatiaea }\end{array}$ & $\begin{array}{l}\text { CANADA, NL, } \\
\text { L'Anse aux } \\
\text { Meadows } \\
51.594895 \mathrm{~N}, \\
55.533285 \mathrm{~W} ; 6\end{array}$ & $\begin{array}{l}\text { bog } \\
\text { Sphagnum }\end{array}$ & $\begin{array}{l}18-S e p- \\
12\end{array}$ & $\begin{array}{l}\text { Andrus Voitk } \\
\text { GNP-064 }\end{array}$ & TUF117357 \\
\hline $\begin{array}{l}\text { MH198210 } \\
\text { UDB032723 }\end{array}$ & $\begin{array}{l}\text { Arrhenia } \\
\text { telmatiaea }\end{array}$ & $\begin{array}{l}\text { CANADA, NL, } \\
\text { Labrador, Trans } \\
\text { Labrador Highway } \\
52.899521 \mathrm{~N}, \\
57.161205 \mathrm{~W} ; 374\end{array}$ & $\begin{array}{l}\text { bog } \\
\text { Sphagnum }\end{array}$ & $\begin{array}{l}\text { 14-Sep- } \\
16\end{array}$ & $\begin{array}{l}\text { Maria Voitk } \\
\text { 16.09.14.av01 }\end{array}$ & $\begin{array}{l}\text { DAOM744398 } \\
\text { TUF117458 }\end{array}$ \\
\hline $\begin{array}{l}\text { MH198214 }{ }^{\mathrm{C}} \\
\text { UDB034619 }\end{array}$ & $\begin{array}{l}\text { Arrhenia } \\
\text { telmatiaea }\end{array}$ & $\begin{array}{l}\text { ESTONIA, Tartu } \\
\text { Co., Puhja Comm. }\end{array}$ & & $\begin{array}{l}16-O c t- \\
86\end{array}$ & $\begin{array}{l}\text { Kuulo } \\
\text { Kalamees }\end{array}$ & TAAM143530 \\
\hline $\begin{array}{l}\text { MH198228 c } \\
\text { UDB034638 }\end{array}$ & $\begin{array}{l}\text { Arrhenia } \\
\text { telmatiaea }\end{array}$ & $\begin{array}{l}\text { SWEDEN, Västra } \\
\text { Götaland Co., } \\
\text { Essunga Comm., } \\
\text { Bäreberg }\end{array}$ & & $\begin{array}{l}\text { 02-Sep- } \\
89\end{array}$ & $\begin{array}{l}\text { Leif \& Anita } \\
\text { Stridvall } \\
\text { LAS89/024 }\end{array}$ & GB-0065942 \\
\hline $\begin{array}{l}\text { MH198184 c } \\
\text { UDB024392 }\end{array}$ & $\begin{array}{l}\text { Arrhenia } \\
\text { telmatiaea }\end{array}$ & $\begin{array}{l}\text { FINLAND, } \\
\text { Varsinais-Suomi, } \\
\text { Vihti, Iso } \\
\text { Sammakko }\end{array}$ & & $\begin{array}{l}08-0 c t- \\
96\end{array}$ & $\begin{array}{l}\text { Ilkka } \\
\text { Kytövuori } \\
96-1308\end{array}$ & H6034872 \\
\hline $\begin{array}{l}\text { MH198186 c } \\
\text { UDB024394 }\end{array}$ & $\begin{array}{l}\text { Arrhenia } \\
\text { telmatiaea }\end{array}$ & $\begin{array}{l}\text { FINLAND, Etelä- } \\
\text { Häme, Lempäälä, } \\
\text { Hietaniemi }\end{array}$ & & $\begin{array}{l}\text { 12-Sep- } \\
87\end{array}$ & $\begin{array}{l}\text { Pertti Salo } \\
8133\end{array}$ & H6042077 \\
\hline $\begin{array}{l}\text { MH198185 c } \\
\text { UDB024393 }\end{array}$ & $\begin{array}{l}\text { Arrhenia } \\
\text { telmatiaea }\end{array}$ & $\begin{array}{l}\text { FINLAND, } \\
\text { Uusimaa, } \\
\text { Kirkkonummi, } \\
\text { Väransby }\end{array}$ & & $\begin{array}{l}26-S e p- \\
93\end{array}$ & $\begin{array}{l}\text { llkka } \\
\text { Kytövuori } \\
93-1524\end{array}$ & H6057157 \\
\hline
\end{tabular}




\begin{tabular}{|c|c|c|c|c|c|c|}
\hline $\begin{array}{l}\text { GenBank } \\
\text { number top } \\
\text { UNITE below }\end{array}$ & $\begin{array}{l}\text { Phylogenetic } \\
\text { ID } \mathrm{b}\end{array}$ & $\begin{array}{l}\text { COUNTRY, } \\
\text { STATE/PROVINCE, } \\
\text { Town, locality } \\
\text { above Coordinates } \\
\text { (degree decimal); } \\
\text { elevation (m asl) } \\
\text { below }\end{array}$ & $\begin{array}{l}\text { Habitat } \\
\text { above, } \\
\text { Substrate } \\
\text { below }\end{array}$ & Date & $\begin{array}{l}\text { Collector } \\
\text { above } \\
\text { Collection } \\
\text { number below }\end{array}$ & $\begin{array}{l}\text { Herbarium } \\
\text { number: Main } \\
\text { above copy } \\
\text { below }\end{array}$ \\
\hline $\begin{array}{l}\text { MH198182 c } \\
\text { UDB024581 }\end{array}$ & $\begin{array}{l}\text { Omphalina } \\
\text { fusconigra } \\
\text { holotype }\end{array}$ & $\begin{array}{l}\text { UK, SCOTLAND, } \\
\text { South Perthshire, } \\
\text { Blair Drummond }\end{array}$ & Sphagnum & $\begin{array}{l}28-S e p- \\
57\end{array}$ & J. Grainger & $\mathrm{K}(\mathrm{M}) 98588$ \\
\hline $\begin{array}{l}\text { MH198193 c } \\
\text { UDB024402 }\end{array}$ & $\begin{array}{l}\text { Arrhenia } \\
\text { telmatiaea }\end{array}$ & $\begin{array}{l}\text { GERMANY, Bayern, } \\
\text { Garmisch- } \\
\text { Partenkirchen, } \\
\text { Fügsee }\end{array}$ & & $\begin{array}{l}22-O c t- \\
95\end{array}$ & $\begin{array}{l}\text { Chistoph } \\
\text { Johannes } \\
\text { Hahn }\end{array}$ & M-0276632 \\
\hline $\begin{array}{l}\text { MH198213 } \\
\text { UDB034618 }\end{array}$ & $\begin{array}{l}\text { Arrhenia } \\
\text { telmatiaea }\end{array}$ & $\begin{array}{l}\text { ESTONIA, Ida-Viru } \\
\text { Co., Sirtsi swamp }\end{array}$ & & $\begin{array}{l}01-\text { Oct- } \\
95\end{array}$ & $\begin{array}{l}\text { Tõnu } \\
\text { Ploompuu }\end{array}$ & TAAM142868 \\
\hline $\begin{array}{l}\text { MH198216 } \\
\text { UDB034621 }\end{array}$ & $\begin{array}{l}\text { Arrhenia } \\
\text { telmatiaea }\end{array}$ & $\begin{array}{l}\text { ESTONIA, Pärnu } \\
\text { Co., Nigula bog }\end{array}$ & & $\begin{array}{l}10-O c t- \\
88\end{array}$ & $\begin{array}{l}\text { Kuulo } \\
\text { Kalamees, } \\
\text { Mall Vaasma }\end{array}$ & TAAM144086 \\
\hline $\begin{array}{l}\text { MH198217 } \\
\text { UDB034622 }\end{array}$ & $\begin{array}{l}\text { Arrhenia } \\
\text { telmatiaea }\end{array}$ & $\begin{array}{l}\text { ESTONIA, Tartu } \\
\text { Co., Ahunapalu }\end{array}$ & & $\begin{array}{l}\text { 09-Nov- } \\
91\end{array}$ & $\begin{array}{l}\text { Kuulo } \\
\text { Kalamees }\end{array}$ & TAAM145308 \\
\hline $\begin{array}{l}\text { MH198215 } \\
\text { UDB034620 }\end{array}$ & $\begin{array}{l}\text { Arrhenia } \\
\text { telmatiaea }\end{array}$ & $\begin{array}{l}\text { ESTONIA, Ida-Viru } \\
\text { Co., Sirtsi swamp }\end{array}$ & & $\begin{array}{l}06-O c t- \\
96\end{array}$ & $\begin{array}{l}\text { Tõnu } \\
\text { Ploompuu }\end{array}$ & TAAM147228 \\
\hline $\begin{array}{l}\text { MH198212 c } \\
\text { UDB034617 }\end{array}$ & $\begin{array}{l}\text { Arrhenia } \\
\text { telmatiaea }\end{array}$ & $\begin{array}{l}\text { CANADA, NL, } \\
\text { Labrador, Trans } \\
\text { Labrador Highway } \\
\text { near Red Bay } \\
51.749723 \mathrm{~N}, \\
56.420208 W ; 27\end{array}$ & $\begin{array}{l}\text { bog } \\
\text { Sphagnum }\end{array}$ & $\begin{array}{l}\text { 07-Sep- } \\
05\end{array}$ & $\begin{array}{l}\text { Tony Wright } \\
\text { 05.09.07.av01 }\end{array}$ & $\begin{array}{l}\text { DAOM744399 } \\
\text { TUF117580 }\end{array}$ \\
\hline U66453 k & $\begin{array}{l}\text { Arrhenia } \\
\text { telmatiaea }\end{array}$ & $\begin{array}{l}\text { GREENLAND, } \\
\text { Disco }\end{array}$ & & & $\begin{array}{l}\text { François } \\
\text { Lutzoni }\end{array}$ & \\
\hline $\begin{array}{l}\text { MH198229 c } \\
\text { UDB034639 }\end{array}$ & $\begin{array}{l}\text { Arrhenia } \\
\text { telmatiaea }\end{array}$ & $\begin{array}{l}\text { SWEDEN, Västra } \\
\text { Götaland Co., } \\
\text { Trollhättan } \\
\text { Comm., } \\
\text { Björndalen }\end{array}$ & & $\begin{array}{l}11-\text { Oct- } \\
97\end{array}$ & $\begin{array}{l}\text { Leif \& Anita } \\
\text { Stridvall } \\
\text { LAS97/113 }\end{array}$ & GB-0065958 \\
\hline $\begin{array}{l}\text { MH198208 c } \\
\text { UDB032193 }\end{array}$ & $\begin{array}{l}\text { Arrhenia } \\
\text { telmatiaea }\end{array}$ & $\begin{array}{l}\text { CANADA, NL, } \\
\text { Labrador, near } \\
\text { Northwest River, } \\
\text { Goose Bay Ski } \\
\text { Club, Loppet trail } \\
\text { 53.33853N, } \\
\text { 60.4497W; } 44\end{array}$ & $\begin{array}{l}\text { bog } \\
\text { Sphagnum }\end{array}$ & $\begin{array}{l}10-S e p- \\
16\end{array}$ & $\begin{array}{l}\text { Chris Deduke } \\
\text { GBHV16B-253 }\end{array}$ & $\begin{array}{l}\text { DAOM744396 } \\
\text { TUF117457 }\end{array}$ \\
\hline \multirow[t]{2}{*}{$\begin{array}{l}\text { MH198209 c } \\
\text { UDB032194 }\end{array}$} & $\begin{array}{l}\text { Arrhenia } \\
\text { telmatiaea }\end{array}$ & $\begin{array}{l}\text { CANADA, NL, } \\
\text { Labrador, Red Bay, } \\
\text { Saddle Island } \\
51.724588 \mathrm{~N}, \\
56.426100 \mathrm{~W} ; 24\end{array}$ & $\begin{array}{l}\text { bog } \\
\text { Sphagnum }\end{array}$ & $\begin{array}{l}\text { 08-Sep- } \\
05\end{array}$ & $\begin{array}{l}\text { Andrus Voitk } \\
\text { 05.09.08.av02 }\end{array}$ & $\begin{array}{l}\text { DAOM744397 } \\
\text { TUF117459 }\end{array}$ \\
\hline & $\begin{array}{l}\text { Arrhenia } \\
\text { telmatiaea }\end{array}$ & $\begin{array}{l}\text { CANADA, NL, Cape } \\
\text { Freels Cemetery }\end{array}$ & $\begin{array}{l}\text { barrens } \\
\text { Sphagnum }\end{array}$ & $\begin{array}{l}22-S e p- \\
21\end{array}$ & $\begin{array}{l}\text { Bill Bryden } \\
\text { 21.09.22.av01 }\end{array}$ & TUF117940 \\
\hline
\end{tabular}




\begin{tabular}{|c|c|c|c|c|c|c|}
\hline $\begin{array}{l}\text { GenBank } \\
\text { number top } \\
\text { UNITE below }\end{array}$ & $\begin{array}{l}\text { Phylogenetic } \\
\text { ID b }\end{array}$ & $\begin{array}{l}\text { COUNTRY, } \\
\text { STATE/PROVINCE, } \\
\text { Town, locality } \\
\text { above Coordinates } \\
\text { (degree decimal); } \\
\text { elevation (m asl) } \\
\text { below }\end{array}$ & $\begin{array}{l}\text { Habitat } \\
\text { above, } \\
\text { Substrate } \\
\text { below }\end{array}$ & Date & $\begin{array}{l}\text { Collector } \\
\text { above } \\
\text { Collection } \\
\text { number below }\end{array}$ & $\begin{array}{l}\text { Herbarium } \\
\text { number: Main } \\
\text { above copy } \\
\text { below }\end{array}$ \\
\hline & $\begin{array}{l}\text { Arrhenia } \\
\text { telmatiaea }\end{array}$ & $\begin{array}{l}\text { CANADA, NL, } \\
\text { Cape Freels }\end{array}$ & $\begin{array}{l}\text { barrens } \\
\text { Sphagnum }\end{array}$ & $\begin{array}{l}22-S e p- \\
21\end{array}$ & $\begin{array}{l}\text { Andrus Voitk } \\
\text { 21.09.22.av02 }\end{array}$ & TUF117941 \\
\hline & $\begin{array}{l}\text { Arrhenia } \\
\text { telmatiaea }\end{array}$ & $\begin{array}{l}\text { CANADA, NL, } \\
\text { Cape Freels }\end{array}$ & $\begin{array}{l}\text { barrens } \\
\text { Sphagnum }\end{array}$ & $\begin{array}{l}22-S e p- \\
21\end{array}$ & $\begin{array}{l}\text { Greg Thorn } \\
\text { 21.09.22.av03 }\end{array}$ & TUF117942 \\
\hline
\end{tabular}

a Arranged by species in alphabetical order. Within each species, collections appear in order of appearance in Fig. 2, followed, where applicable, by sequenced specimens not in Fig. 2, and one unsequenced specimen.

b Type collections, in boldface, bear their basionym, all others identified by their current names.

c Sequence generated at Tartu University.

d Sequence generated at Bridgwater College

e Logoimage for Foray Newfoundland \& Labrador.

${ }^{\mathrm{f}}$ Specimen all used up in sequencing.

g Sequence not shown in Fig. 2.

h Specimen failed to yield amplifiable DNA.

i Sequence generated at the Field Museum,

j Collection lost in transit on return from sequencing.

i Sequence generated by Lutzoni (1997).

\section{Phylogeny}

The studied collections produced two singleton collections and four clades within Arrhenia, as well as one clade that fell in Omphalina, code named here "OPCUS", acronym for Omphalina pyxidata complex, unidentified species (Figs. 1, 2). Two scaly-capped sphagnicolous species arose as sister clades from the most ancestral lineage in the arm leading to Arrhenia, while the two smooth-capped species emerged later. A more detailed look at these four species (Fig. 2) reveals that the epitype of Ag. gerardianus (NYSd 4725) fell into one scaly-capped clade, giving it its name, and Bigelow's holotype of $C$. gerardiana var. fusca fell into the other, which we describe as a new species, Arr. bigelowii. Berkeley \& Cooke's holotype for Ag. telmatiaeus and Orton's holotype for $O$. fusconigra fell into a clade of dark smooth-capped obligatory sphagnicolous basidiomes, making 0 . fusconigra a later synonym of $\mathrm{Ag}$. telmatiaeus. We transfer the latter to Arrhenia as Arr. telmatiaea (Berk. \& Cooke) Voitk \& I. Saar, comb. nov. The remaining clade we identify as Arr. philonotis, and declare a neotype from Germany for the species. Two singleton specimens, both identified in the field as "Omphalina sphagnicola" and labeled 'sphagnicola' on Fig. 1, fell elsewhere within Arrhenia. The name was one primarily, but not exclusively, used for scaly-capped sphagnicolous arrhenias at the time; because we have very little tissue left, and lack notes or photographs for these collections, including whether they were 
sphagnicolous or scaly, and have not collected them again on return trips to the same sites, we show them but do not treat them here.

\section{Macromorphology}

Phylogenetic clades correlated with morphological characters. Both species in the earlier diverging lineage have pilei usually under $20 \mathrm{~mm}$ in diameter, covered to a various degree by radially arranged darkening scales with upturned tips (Fig. 3a-c; 4a-e). Macroscopically the upturned scale tips make distinction between scaly- and smooth-capped specimens easy in vivo, but the difference is not readily apparent after drying because often the upturned tips are not obvious microscopically even after rehydration.

About $10 \%$ or less of Arr. bigelowii specimens undergo a marked darkening reaction (Fig. $4 \mathrm{~b}, \mathrm{~d}$ ); the species as a whole may be a little darker than Arr. gerardiana, but lighter specimens are indistinguishable from the latter (Fig. 4a, e). Occasionally the scales disappear from some very mature (caps over $20 \mathrm{~mm}$ in diameter) specimens of Arr. gerardiana (Figs. 3d; 4f), possibly through loss of the outer pileipellis layer, making such specimens difficult to distinguish from similar smooth-capped taxa, particularly OPCUS. The two smooth-capped species (Fig. 5) in the more derived lineage, have mature cap diameters over $20 \mathrm{~mm}$, at times reaching over 35 . Rudimentary outlines of similar scales can be seen in the pileipellis of Arr. telmaiaea (Fig. 3f, g), which remain adpressed without tips rising up. Fine adpressed hairs outlining similar radially arranged scale shapes with centrifugal points are spread sparsely about the cap of Arr. philonotis, occasionally rising to form scattered thin hairs, better visible with magnification (Fig. 3e).

The descriptions of both Agaricus telmatiaeus and Omphalina fusconigra fit the

dark smooth-capped species (Fig. 5c-e). Orton's description of the cap texture was confirmed by our observations: the cap of 17 basidiomata is well seen on photos of eight collections confirmed by molecular studies to be conspecific with $O$. fusconigra. All caps are smooth, but one rather old basidioma reveals scurfy-flocculose texture limited to the depth of the navel. In other words, this is not a scaly-capped species, but just as Orton described, a smooth-capped species, which only on occasion ( $<6 \%$ of basidiomata in our experience) develops wrinkling irregularity in the depth of its navel. The protologue for Arr. philonotis fits the specimens in its clade (Fig. 5a, b). Most of the time these two smooth-capped species are relatively easy to separate because one has very dark caps, the other lighter, but both have a wide spectrum of darkness (Fig. 3f, g for Arr. telmatiaea and $5 \mathrm{a}, \mathrm{b}$ for Arr. philonotis), making confusion possible. OPCUS (Fig. 5f, g) has the largest basidiomata, occasionally over $35 \mathrm{~mm}$ cap diameter, a smooth cap, and a colour that leans more toward reddish than greyish brown. In Sphagnum, it can add to the confusion of identifying these species.

\section{Micromorphology}

All species have clamp connections through all tissues, primarily 4-spored basidia, and lack cystidia. Basidiospores of Arr. telmatiaea are the smallest, averaging $7.3 \times 4.7 \mu \mathrm{m}$, separating it from Arr. philonotis, averaging $8.7 \times 5.6 \mu \mathrm{m}$, most of the time. Basidiospores of the scaly-capped species are longer and narrower than the spores of the smoothcapped species, but do not separate as well from each other (Fig. 6). Those of Arr. gerardiana are smaller than those of its sometimes darkening sister, but the majority cluster in the overlapping section, making measurement a helpful differentiator only at the extremes of their combined range. The pileipellis of all four species consists of repent clamped hyaline cells with a modest to moderately copious amount of incrusted brownish pigment, some evident as small plates on the cell wall (Fig. 7a-d). Incrustation is most obvious in the scaly-capped species and least in the hyaline hyphae of Arr. telmatiaea, where incrustation was subtle and required time to find. The scales of Arr. bigelowii 
and Arr. gerardiana resemble each other, fascicles of thick pileipellis hyphae turning to project upwards to form a visible acute tipped scale (Fig. 7e, f), with unpigmented clear rounded terminal cells.

\section{Ecology and distribution}

Three species were observed to be obligate sphagnophiles. Of these, the two scaly-capped species, Arr. gerardiana and Arr. bigelowii, were sympatric, on occasion found in the same bog at the same time. The dark smooth-capped Arr. telmatiaea was also an obligate sphagnophile. The other smooth-capped species, Arr. philonotis was a facultative shpagnophile, growing in bogs, heaths, moors and other exposed places, among Sphagnum, but at least equally often with other mosses. OPCUS was collected from bogs with Sphagnum (Fig. $5 \mathrm{~g}$ ) or heaths and moors with other mosses, but was most common in grasslands with low moss (Fig. 5f). All four species of Arrhenia were distributed through both Europe and North America (including Greenland). In NL smooth-capped arrhenias were more northerly, found in Labrador, the Great Northern Peninsula, and along the northern east coast, bathed by the Labrador Current. The scalycapped species seemed to extend throughout the province. OPCUS shared the same distribution with the scaly-capped species in NL, and matching GenBank sequences (not shown in our tree), confirm its presence in Europe.

\section{Taxonomy}

Arrhenia bigelowii Voitk, Lickey \& I. Saar, sp. nov. Fig. 3a; 4a-d; 7a, e, f.

MycoBank MB827069

Typification: CANADA, NL, Rocky Harbour bog, $49.577694^{\circ} \mathrm{N}, 57.898916^{\circ} \mathrm{W}, 35 \mathrm{~m}$ asl, bog in Sphagnum, 5 Jul 2005, leg. Andrus Voitk 04.07.05.av03. (DAOM744391, holotype)

= Clitocybe gerardiana var. fusca H.E. Bigelow, Mycologia 50(1):401. 1958. USA, MI, Luce Co., Pike Lake, bog in Sphagnum, 11 Sep 1953, leg. Alexander H. Smith, 42574 (MICH 10143, varietal

holotype). Ibid; a solitary partial pileus donated from the holotype collection MICH 10143 to TUF, and accessioned as an isotype (TUF117871! varietal isotype, GenBank MH473348).

Etymology: Bigelowii honours the American mycologist Howard Elson Bigelow, the first to publish this entity as a separate taxon.

Diagnosis. Scaly-capped obligate sphagnopilic denizen of temperate to subarctic raised bogs, pileus usually under 20 $\mathrm{mm}$ diameter, occasionally darkens. Pinnate scales distinguish it from smooth-capped sphagnophilic arrhenias; in addition to being somewhat darker most of the time, it can be separated from the other scaly-capped sphagnicolous species, Arr. gerardiana, by an occasional darkening reaction, tendency for more inverted bowl shaped pilei, longer spores, and diagnostic ITS sequence.

Capsule varietal isotype description. Studied material was approximately one-half of a dried pileus with gills attached, approx. $1.5 \mathrm{~cm}$ in diam. Cap striate, umbilicate. Microscopic examination of a squash section of gill revealed elongate elliptical spores 6.1-11.4 × 3.2-4.9 $\mu \mathrm{m}$ (ave. 8.4 × 4.0, Q 1.7-2.5, ave 2.1, n=22 spores). No cystidia, but clamp connections readily evident. Four-spored club-shaped basidia, approx. size $34 \times 7 \mu \mathrm{m}$. Cap structure not examined.

Macromorphology (Fig. 3a; 4a-d): Basidioma: Brown, stipitate, about 8-33 mm tall, in Sphagnum. Uncommonly becomes dusky, with various degrees of black and grey adding to or covering the brown colouration. Stimulus for this change not known. Pileus: 4-24 mm diameter, usually deeply umbilicate and often shaped like an inverted bowl, edges becoming more plane, then crenulate with age, translucently striate, smooth, but covered with somewhat 
concentric radially arranged scales with darker brown, burr-like, uplifted, centripetally narrowing ends. Narrow brown radial bands over lamellae and lamellulae, alternating with wider, tan intervening bands; the latter become sulcate with time, giving the cap a radially ribbed appearance. Rim of edge darkens with time. Lamellae: moderately spaced, smooth edged, deeply decurrent, with usually three intervening, small lamellulae; developing a few low crossveins beyond maturity, forking very rare; light brown, developing darker edge. Stipe: $2-5 \times 10-23 \mathrm{~mm}$, cylindric, straight; becoming somewhat hollow; minutely tomentose, glabrescent with age, brown, sparse white tomentum at base. Context: whitish, odor unremarkable.

Micromorphology: Basidiospores (369 spores, 18 basidiomata, 18 collections, 3 observers) 6.1-17.0 $\times 3.0-6.1 \mu \mathrm{m}$, ave. $10.3 \times 4.6 \mu \mathrm{m}$, elongate elliptical, $\mathrm{Q}=1.6-3.3$, ave. 2.3 , content homogeneous, some variation in size and shape between individual basidiomata (Fig. 6). Basidia 29-39 × 6.2-8.8 $\mu \mathrm{m}$, ave. $35 \times 7.4 \mu \mathrm{m}$; mostly four-spored with occasional two-spored; clavate, hyaline. No pleuro-, cheilo- or caulocystidia, but terminal hyphal cells protrude from stipe as small hairs. Pileipellis a cutis with thin-walled, clamped hyphae, 3.5-13.5 $\mu \mathrm{m}$ wide, hyaline to brownish, with moderately incrusted brown pigment, superficial layers forming small plates on the cell surface (Fig. 7a). Scale tips end with gently swollen rounded unpigmented clear cells (Fig. 7e, f). Clamp connections in all tissues.

Habitat: Open raised Sphagnum bogs in groups of 1-6 separate basiomata, attached to living Sphagnum with white mycelial tomentum, associated with various bog plants such as Vaccinium oxycoccos L., V. macrocarpon Aiton, Rubus chamaemorus L., Empetrum nigrum L., Andromeda polifolia L., and various bog orchids, reeds and grasses, June-Sept, most plentiful in July. May be found in the same bog at the same time as Arr. gerardiana.

Distribution: Known from North America and Europe; in NL found throughout the province, more common on the Island.

Additional specimens examined: See Table 3.

Comments: Often indistinguishable from Arr. gerardiana: darkening reaction often absent and pileus becomes more plane with age. Although statistical analysis shows that the spore size difference is highly significant (for $Q, t=-6.31$; d.f. $=788 ; p<0.001)$, spore sizes of the two species overlap sufficiently to make this character useful only when the averages occupy the extremes of their respective range. We elected to describe it as a new species because we wished to provide a fully examined and robust type collection for posterity. Bigelow (1958) described the growth pattern as "scattered", typical of scaly-capped sphagnicolous arrhenias, whose individual organisms usually produce 1-2 fruiting bodies at any one time, and seldom more than six. Smith's holotype collection of $C$. gerardiana var. fusca consists of more than 30 basidiomata, and must sample several individual organisms. Because both Arr. bigelowii and Arr. gerardiana are macro- and micromorphologically very similar, known to occur in the same bog at the same time, a multi-individual collection increases the likelihood of containing both species. We have not examined the entire collection, but only a cap fragment, from which tissue has been removed for both microscopy and molecular studies. This seems inadequate to designate as lectotype, when an abundance of tissue remains in the holotype for the variety, and typifying the species with an ample and fully examined collection seems the more prudent choice. In addition, given the considerable confusion caused by the name "fusconigra" in the past, avoiding a name from the same root seems advantageous, all the moreappropriate because this gives an opportunity to honour Bigelow, the first to recognize this species a new taxon.

Arrhenia gerardiana (Peck) Elborne, Funga Nordica: 913. 2008. FIG. 3b-d; 4 e,f ; 7g, h

Basionym: Agaricus (Clitocybe) gerardianus Peck, Bull. Buffalo Soc. Nat. Sci. 1: 46. 1873.

Page 25/45 
Typification: USA: NEW YORK, Ulster Co, New Paltz, June, 1873 (approx.), in "sphagnous marshes", leg. C.H. Peck. (NYSf 1339.1-4! Lectotype, here designated; MBT10005613). USA: NEW YORK, Rensselaer Co., Sand Lake, June, 1873 (approx.), in "sphagnous marshes", leg. C.H. Peck. (paratype, NYSf 1340, not seen, possibly lost). USA: NEW YORK, Essex County, North Elba, Mt. Marcy, June, no year, leg. \& det. C.H. Peck (as Omphalia gerardiana) M212-S294000(7794) (NYSd 4725.1!, epitype, here designated. Mycobank type number MBT10005614).

Capsule lectotype description. The lectotype collection NYSf 1339.1-4, consists of four dried dark brown basidiomata glued to sheets (.1 and .4 further secured with cloth tape), reasonably intact with pieces of stipe and pileus missing. Tallest approx. $5.5 \mathrm{~cm}$ high (stipe base to top of upturned cap edge), with widest cap diam. approx. $1.2 \mathrm{~cm}$. Caps striate, umbilicate to funnel-shaped. Microscopic examination of a squash section of gill ( $3 \% \mathrm{KOH})$ from each of the four basidiomata revealed pip-shaped to elliptical spores 6.8-12.4 × 3.4-5.1 um (ave. 9.2 × 4.2, ave Q = 2.2, $n=100$ spores). No cystidia. Clamp connections throughout all tissues. Four-spored (rarely two) club-shaped basidia, approx. size $24 \times 7 \mu \mathrm{m}$. Cap structure not examined.

Capsule epitype description. The epitype collection NYSd 4725, consisted of ten relatively intact dried dark brown basidiomata, most taped or glued to sheets, with parts missing, and additional pieces with most of the basidiomata missing. Tallest approx. $5.5 \mathrm{~cm}$ high (stipe base to top of upturned cap edge), with widest cap diam. approx. $1.2 \mathrm{~cm}$. Caps striate, umbilicate to funnel-shaped. The epitype was selected and designated NYSd 4725.1. Microscopic examination of a squash section of gill revealed pip-shaped to elliptical spores 7.9-11.5 × 3.3-4.2 $\mu \mathrm{m}$ (ave. 9.8 × 3.8, ave $\mathrm{Q}=2.6, \mathrm{n}=20$ spores). No cystidia. Clamp connections throughout all tissues. Four-spored club-shaped basidia, approx. size $24 \times 7 \mu \mathrm{m}$. Cap structure not examined.

Macromorphology (Fig. 3b-d; Fig. 4e, f): Basidioma: Brown, stipitate, about 10-35 mm tall, in Sphagnum. Pileus: 5$25 \mathrm{~mm}$ diameter, usually deeply umbilicate, edges curved down somewhat at the edges, but rarely assuming an inverted bowl shape, becoming plane, then crenulate with age, translucently striate, smooth, but covered with somewhat concentric radially arranged scales with darker brown, burr-like, uplifted, narrow distal ends. These scales seem to recede with age, and occasional very mature specimens with large caps may have no distinctive scales (Fig. 3d; 4f). Narrow brown radial bands over lamellae and lamellulae, alternating with wider, tan intervening bands; the latter become minimally sulcate with time. Rim of edge darkens with time. Lamellae: moderately spaced, smooth edged, deeply decurrent, usually with three intervening, small lamellulae; developing a few low crossveins beyond maturity, forking very rare; light brown, developing darker edge. Stipe: 2-5 × 10-25 mm, cylindric, straight; becoming somewhat hollow; minutely tomentose, which may disappear with age, brown with sparse white tomentum at base. Context: whitish, odor unremarkable.

Micromorphology: Basidiospores (443 spores, 20 basiomata, 17 collections, 3 observers) $6.2-12.9 \times 2.8-5.6 \mu \mathrm{m}$, ave. $8.9 \times 4.2 \mu \mathrm{m}$, pip-shaped to elliptical, $\mathrm{Q}=1.3-3.2$, ave. 2.2, content homogeneous, some variation in size and shape between individual basiomata (Fig. 6). Basidia $28(21-35) \times 6.8(5.6-9.0) \mu \mathrm{m}$; mostly four-spored with occasional two-spored; clavate, hyaline. No pleuro-, cheilo- or caulocystidia, but terminal hyphal cells protrude from stipe as small hairs. Pileipellis a cutis with thin-walled, clamped hyphae, 2.5-9.5 $\mu \mathrm{m}$ wide, hyaline to brownish, with moderately incrusted brown pigment, superficial layers forming small plates on the cell surface (Fig. 7b). Scale tips end with gently swollen rounded unpigmented clear cells (Fig. 7g, h). Clamp connections in all tissues.

Habitat: Open raised Sphagnum bogs in groups of 1-6 separate basidiomata, attached to living Sphagnum with white mycelial tomentum, associated with various bog plants such as Vaccinium oxycoccos, V. macrocarpon, Rubus 
chamaemorus, Empetrum nigrum, Andromeda polifolia, and various bog orchids, reeds and grasses, June-September, most plentiful in July. May be found in the same bog at the same time as Arr. bigelowii.

Distribution: Europe and North America; suspect Holarctic distribution. In NL, throughout the province.

Additional specimens examined: See Table 3.

Comments: Its cap, scaly throughout, sets it apart from smooth-capped sphagnicolous omphalinoids. This character may be lost in very few overly mature and large specimens, requiring microscopic examination to distinguish them from Arr. philonotis, OPCUS, or even lighter-coloured Arr. telmatiaea. Differs from Arr. bigelowii by its more plane pilei, by not turning grey to black in response to unknown stimuli, and shorter spores; these characters are not always evident.

Peck (1873) mentioned two collections in his description of Agaricus gerardianus (NYSf 1339 and NYSf 1340), which become syntypes because he did not designate either as holotype. NYSf 1340 is presumed lost; we designated the remaining collection (NYSf 1339.1-4) with its four basiomata, as lectotype for the species. Comparison of spore size has allowed us to conclude that the lectotype is conspecific with collection NYSd 4725, identified by Peck as $A g$. gerardianus. Because the lectotype did not yield DNA, but NYSd 4725 did, we designated the latter as epitype for Arrhenia gerardiana, thus defining the clade in which it resides as that species.

Arrhenia philonotis (Lasch) Redhead, Luzoni, Moncalvo \& Vilgalys, Mycotaxon 83: 48. 2002. Fig. 3e; 5a,b; 7c

MycoBank MB374174

Basionym: Agaricus philonotis Lasch, Linnaea 3:394. 1828.

Typification: Holotype probably lost. GERMANY, Baden-Württemberg, Schwarzwald, Ks. Breisgau-Hochschwarzwald, Hinterzarten, Hinterzartener Moor, MTB/Q 8014/4, Sphagnum, 29 Jul 1984, leg. D. Laber, (Neotype, here designated: KR-0003880! Mycobank type number MBT10005615).

Capsule neotype description. The neotype collection KR-0003880, is fragmented with no completely intact basiomata. Larger pieces are parts of at least 4-5 pilei and twice that number of stems, suggesting that the original collection may have consisted of around 8-10 basiomata. Although fragmented, there is a generous amount of material, which otherwise seems to be in good shape. Small strands of moss are seen, including at least one stem attached to what seems to be Sphagnum. By extrapolation, the tallest dried basioma is approx. $5 \mathrm{~cm}$ high (stipe base to top of upper cap edge), with widest cap diam. approx. $2.5 \mathrm{~cm}$. Caps smooth, umbilicate. Microscopic examination of a squash section of gill revealed pip-shaped to elliptical spores 7.7-10.4 × 5.2-7.6 $\mu \mathrm{m}$ (ave. $8.9 \times 5.8$, ave $\mathrm{Q}=1.5, \mathrm{n}=20$ spores). No cystidia. Clamp connections throughout all tissues. Four-spored club-shaped basidia, approx. size $24 \times 7$ $\mu \mathrm{m}$. Cap structure not examined.

Macromorphology (Fig. 3e; 5a,b): Basidioma: Brown, stipitate, about 10-38 mm tall, in heaths, bogs and moors with Sphagnum or other moss. Pileus: 5-30 mm diameter, umbilicate, edges curved down becoming plane and crenulate with age, translucently striate, smooth, often covered with thin, fibrillose, adpressed, flat scales, whose tips may become slightly uplifted as scattered thin hairs, denser in the umbilicus. Narrow brown radial bands over lamellae and lamellulae, alternating with wider, tan intervening bands; the latter may become sulcate with time, giving the cap a radially ribbed appearance. Rim of edge darkens with time. Lamellae: moderately spaced, smooth edged, deeply decurrent, with 3-5 intervening, small lamellulae; may develop a few low crossveins beyond maturity, forking very 
rare; light brown, developing darker edge. Stipe: 2-5 × 10-25 mm, cylindric, straight; becoming somewhat hollow; minutely tomentose, glabrescent, brown with sparse white tomentum at base. Context: whitish, odor unremarkable.

Micromorphology: Basidiospores (102 spores, 5 basidiomata, 5 collections, 2 observers) 6.6-10.9 $\times 4.2-7.7 \mu \mathrm{m}$, ave. $8.7 \times 5.6 \mu \mathrm{m}$, pip-shaped to elliptical with $\mathrm{Q}=1.2-2.1$, ave. 1.6 , content homogeneous, some variation in size and shape between individual basiomata (Fig. 6). Basidia $29.6(23.3-35.2) \times 7.8(6.1-10.2) \mu \mathrm{m}$; mostly four-spored with occasional two-spored; clavate, hyaline. No pleuro-, cheilo- or caulocystidia, but terminal hyphal cells protrude from stipe as small hairs. Pileipellis a cutis with thin-walled, clamped hyphae, 2.0-11.0 $\mu \mathrm{m}$ wide, hyaline to brownish, with moderately incrusted brown pigment, superficial layers forming small plates on the cell surface (Fig. 7c). Clamp connections in all tissues.

Habitat: Barren moors, heaths, fens, raised Sphagnum bogs in groups of 1-6 separate basidiomata, either with Sphagnum or other moss. Associated with various heath plants such as Vaccinium oxycoccos, $V$. macrocarpon, Rubus chamaemorus, Empetrum nigrum, Andromeda polifolia, reeds and grasses, June-September, most plentiful in August.

Distribution: Known from North America and Europe; Holarctic distribution suspected; not as southerly as the scalycapped species, in NL so far known only from Labrador.

Additional specimens examined: See Table 3.

Comments: Basiomata resemble those of the scaly-capped species, but are readily distinguished by their obviously smooth caps of somewhat greater diameter, and light colour tending more to greyish brown. Distinguished from Arr. telmatiaea by its lighter hue and from OPC by its greyish rather than reddish brown hues and broader spores.

Arrhenia telmatiaea (Berk. \& Broome) Voitk \& I. Saar, comb. nov. Fig. 3f,g; 5c,d,e; 7d

MycoBank MB842881

Basionym: Agaricus telmatiaeus Berk. \& Cooke. Illustrations of British fungi (Hymenomycetes). Williams and Norgate, London. 2: pl 240. UK, England, Yorkshire Co., Scarboro, 2 Nov 1882, leg. G. Massee (NY12555, holotype!)

= Arrhenia fusconigra (P.D. Orton) P.A. Moreau \& Courtec., Documents Mycologiques 34 (135-136): 48 (2008).

Basionym: Omphalina fusconigra P.D. Orton, Transactions of the British Mycological Society 43(2): 335.1960. MycoBank MB518174. UK, Scotland, South Perthshire, Blair Drummond, 28 Sep 1957, leg. J. Grainger (K(M)98588! holotype!).

Capsule holotype description. The holotype collection NY12555, consisted of eight relatively intact dried dark brown basidiomata with adherent Sphagnum, most taped or glued to sheets, with parts missing. Tallest approx. $4.8 \mathrm{~cm}$ high (stipe base to top of cap), with widest cap diam. approx. $3.8 \mathrm{~cm}$. Caps striate, umbilicate. Microscopic examination of a squash section of gill revealed pip-shaped to elliptical spores 6.0-8.1 × 3.9-5.8 $\mu \mathrm{m}($ ave. 7.2 $\times 4.7, \mathrm{Q}=1.3-1.8$, ave $1.5, n=30$ spores). No cystidia. Clamp connections throughout all tissues. Four-spored club-shaped basidia, approx. size $26 \times 7 \mu \mathrm{m}$. Cap structure not examined.

Macromorphology (Fig. 3f,g; 5c,d,e) Basidioma dark brown, usually almost blackish, stipitate, about 10-40 mm tall, in Sphagnum. Pileus: 6-32 mm diameter, usually deeply umbilicate, edges curved down in a pronounced arc, becoming plane and then funnel-shaped with age, translucently striate, smooth, with occasional fine, floccules in the umbilicus. Usually dark brown verging on black, but occasionally may remain mostly brown; dark, narrow, radial bands over 
lamellae and lamellulae, alternating with somewhat lighter deep brown bands; hygrophanous. Rim of edge darkens with time. Lamellae: closely spaced, smooth edged, deeply decurrent, with usually 5-7 intervening lamellulae; forking very rare; medium to dark brown, edge darker. Stipe: 2-6 × 10-28 mm, cylindric, straight; becoming somewhat hollow; minutely tomentose, glabrescent, concolorous with pileus with sparse white tomentum at base. Context: lighter brown, odor unremarkable.

Micromorphology: Basidiospores (241 spores, 12 basiomata, 12 collections) $5.3-11.3 \times 3.3-6.6 \mu \mathrm{m}$, ave. $7.3 \times 4.7$ $\mu \mathrm{m}$, pip-shaped to elliptical, $\mathrm{Q}=1.2-2.2$, ave. 1.6, content homogeneous, some variation in size and shape between individual basiomata (Fig. 6). Basidia 27.6 (21.8-31.6) × 7.1 (5.5-8.6) $\mu \mathrm{m}$; mostly four-spored with occasional twospored; clavate, hyaline. No pleuro-, cheilo- or caulocystidia, but terminal hyphal cells protrude from stipe as small hairs. Pielipellis a cutis with thin-walled, clamped hyphae, 3.5-9.0 $\mu \mathrm{m}$ wide, hyaline to brownish, with sparsely to moderately incrusted brown pigment, superficial layers at times forming small plates on the cell surface (Fig. 7d). Clamp connections in all tissues.

Habitat: Open raised Sphagnum bogs in groups of 1-6 separate basiomata, attached to living Sphagnum with white mycelial tomentum, associated with various bog plants such as Vaccinium oxycoccos, V. macrocarpon, Rubus chamaemorus, Empetrum nigrum, Andromeda polifolia, reeds and grasses, July-September, most plentiful in August. May be found in the same bog at the same time as other northern species.

Distribution: Known from North America and Europe; suspected Holarctic distribution; in NL not as southern as the scaly-capped species, so far known only from Labrador, the Great Northern Peninsula, and the northern east coast.

Specimens examined: See Table 3.

Comments: Its obviously and relatively even dark colour distinguishes it from the other smooth-capped sphagnicolous species, Arr. philonotis, but on occasion may be more dark brown than near-black, requiring microscopic examination to confirm identification. Omphaliaster borealis (M. Lange \& Skifte) Lamoure-not recorded in NL to date-is macroscopically very similar, also occurring in northern or alpine raised Sphagnum bogs, but can be distinguished by its globose, spinulose spores (Vašutová et al. 2013).

OPCUS (Omphalina pyxidata complex, unidentified species) Fig. 5f,g

Macromorphology: Basidioma: Brown, stipitate, about 10-44 mm tall, with various mosses in grassland, moor, fen, as well as bog with Sphagnum. Pileus: 8-40 mm diameter, umbilicate, downcurved edges quickly becoming plane, then upturned and crenulate with age, translucently striate but hygrophanous and opaque when dry, smooth, covered with sparse, thin, fibrillose, adpressed, flat scales. Narrow brown or reddish brown radial bands over lamellae and lamellulae, alternating with wider, tan intervening bands. Red more noticeable if opaque. Lamellae: moderately spaced, smooth-edged, deeply decurrent, with 3-5 intervening, small lamellulae; develops low crossveins beyond maturity, forking very rare; very light off-white, contrasting markedly with darker stem and cap. Stipe: 2-7 × 10-38 $\mathrm{mm}$, cylindric, straight concolorous with cap, sparse white tomentum at base. Context: whitish, odor unremarkable.

Micromorphology: Basidiospores (128 spores, 5 sporocarps, 5 collections) $6.3-9.1 \times 3.6-5.1 \mu \mathrm{m}$, ave $7.8 \times 4.4 \mu \mathrm{m}$, elliptical, $\mathrm{Q}=1.3-2.1$, ave. 1.8. Basidia four-spored, clavate, hyaline (Fig. 6). No pleuro-, cheilo- or caulocystidia. Clamp connections in all tissues.

Habitat: Grows with mosses in grassland, fens, moors and bogs; usually 1-4 basidiomata in bogs, but troops of 20 or more in grassland. Season: June-Sept, most plentiful Jul-Aug. Found in the same bog at the same time as the other species. 
Distribution: Throughout NL; North America and Europe.

Specimens examined: See Table 1.

Comments: Larger than the arrhenias, often reddish, not greyish, but otherwise similar to Arr. philonotis, but with narrower spores.

Dichotomous key to the species of sphagnicolous omphalinoids of NL

1a. Granular green lichen thalli at base of stem and lack of clamp connections Lichenomphalia

1b. No thalli at base of stem, and clamp connections 2

2a. Cap scaly with raised, pointed, often darkened scale tips 3

2b. Cap smooth (may be minutely wrinkled, irregular or wooly, or may have adpressed scales, but not with raised scale tips) 4

3.a May undergo significant darkening reaction, spores 6.1-17.0 × 3.0-6.1 $\mu \mathrm{m}$, ave. $10.3 \times 4.6 \mu \mathrm{m}$ Arrhenia bigelowii

3b. No darkening reaction, spores $6.2-12.9 \times 2.8-5.6 \mu \mathrm{m}$, ave. $8.9 \times 4.2 \mu \mathrm{m}$ Arrhenia gerardiana.

4a. Medium ( \pm reddish) brown, mature cap around $30 \mathrm{~mm}$ diameter, spores $<5 \mu \mathrm{m}$ wide, distribution throughout NL OPCUS

4b. Not as above 5

5a. Dark brown, nearly black, northerly distribution, obligate sphagnophile, spores 5.3-11.3 × 3.3-6.6, ave. 7.3 4.7 Arrhenia telmatiaea

5b. Medium to light grey-brown, northerly distribution, facultative sphagnophile, spores $6.6-10.9 \times 4.2-7.7$, ave. $8.7 \times$ 5.6 Arrhenia philonotis

\section{Discussion}

Sphagnicolous omphalinoid arrhenias have been ignored in the era of molecular studies: in 2006, when we first began this investigation, we found only two sequences in GenBank identified with one of the names in our original list, one collection each. Because we have a large number of collections from a wide geographic range (Table 3) the likelihood of finding additional sphagnicolous arrhenias in accessible bogs of Europe or North America is low, but the existence of uncommon sphagnicolous species is possible in remote habitats outside usually surveyed regions. For example, in a report of 32 Nordic sphagnicolous agarics, Lange \& Lange (1982) reported two very dark scaly-capped sphagnicolous specimens from Greenland that they suspected might represent an unknown species. Spore measurements place them just outside the range of species in our study. As mentioned, we encountered two singletons identified as "Arrhenia sphagnicola", which we excluded from the current study due to lack of photograph or detailed notes. After concluding our study, we encountered another singleton in Sphagnum that we were unable to identify or match to any known name (Voitk \& Burzynski 2018). Extraction of DNA failed, so it was not incorporated into this study. We mention these collections to note that the possibility of finding additional sphagnicolous species in less commonly explored regions may not be exhausted. 
Our molecular studies identified four species, confirming the impression expressed in the Introduction that few species were involved. Once clades were circumscribed phylogenetically, we could examine them retrospectively for identifying and differentiating characters. Thus, molecular studies provided the first opportunity to seek morphologic and ecologic characters that best match protologues and original material of available names. Before molecular studies, in the last seven decades leading students of these taxa used at least eight names for four species, so that no matter which names are used, accommodation and change will be required. Current uncertainty in the community is well illustrated in an on-line discussion (https://mushroomobserver.org/observer/show_observation/89590, last accessed Mar 31, 2021): for a single collection, knowledgeable mycophiles considered four epithets, weighing three of them almost equally. Lücking (personal communication) states that philonotis is the commonest epithet applied to sphagnicolous arrhenias in European herbaria, which is disproportional to its prevalence among the four taxa, suggesting that the name has been applied to more than one species. Such lack of consistency means that any circumscription of species concepts will require significant change. In this situation, any system providing objective identification characters fixed to types would be welcome, with little convincing reason to prefer names other than those with the best fit to original material.

Two characters have been particularly troublesome for nomenclatural consistency in the past. The first is lack of an early name for the common scaly-capped sphagnicolous arrhenias, resulting in the application of incompatible epithets like tigrinus and affricatus to this group, or overinterpreting descriptions of cap vestiture in an effort to shoehorn the description to fit "scaly", e.g. interpreting Lasch's "leviter tomentosus" for Ag. philonotis as scaly instead of sparsely hairy, taking an occasional wrinkled umbilicus of $O$. fusconigra to mean a consistently scaly-cap, or equating Berkeley's description of minute cap surface irregularity of Ag. sphagnicola with the rough scaliness of sphagnicolous arrhenias. The second confusing character is dark hue. Fries contributed to this by synonymizing his Ag. oniscus with a light species in his protologue (Fries 1818) and sanctioning work (Fries 1821), but later (Fries 1867) applying the name to a different and dark species, causing the name to be applied to dark arrhenias (Voitk 2021). Variability of the truly dark species, Arr. telmatiaea, from opaque near-black (Fig. 3g; $5 \mathrm{~b}$ ) to translucently striate dark brown (Fig. 5c, d), lightening even to tan on drying, increases the opportunity to confuse it with a darker specimen of Arr. philonotis (Fig. 5b), desquamated older Arr. gerardiana (Fig. 3d; 4f), or the unexpected OPCUS. In addition, the ill-understood darkening reaction, sometimes extreme (Fig. 4b, d), of Arr. bigelowii adds further confusion to these two taxa, compounded by the confusion over which is scaly and which is not.

Spore size is genetically determined, and functioned as a reliable indicator of genetic lineage before the DNA era. Our four clades separated into three groupings by spore size (Fig. 6, Fig. 8a): one for each of the two smooth-capped species and one larger grouping for the two scaly-capped ones, whose constituent members proved difficult to separate from each other due to a large area of overlap. To get an idea of the consistency of name use by the major pre-DNA workers cited in the Introduction we plotted the spore size ranges for our four species (Fig. 8a), and then superimposed the spore measurements reported for each name by the same leading workers (Fig. 8). Full ranges, including extremes, were used for each, save for Lange \& Lange (1982), who reported the range of average values. The overall pattern was reasonably similar to ours: a cluster around each of the two smooth-capped species, and one covering both scaly-capped ones (Fig. 8b), which suggests that the same four species were studied. Fig. 8c-j shows spore sizes reported for each name used, distributing the eight names among half that number of species.

The epithets most often applied to this group by the selected authors were philonotis and oniscus, both used in eight studies. Both Arr. telmatiaea and Arr. philonotis are smooth-capped cold temperature species, far less common than their scaly-capped relatives. Their frequency in these studies probably reflects the habitats of interest to the workers more than the relative prevalence of the species. Interestingly, spore measurements of seven of the eight species to which oniscus was applied (Fig. 8d), fit with those of Arr. telmatiaea; the measurements reported by Kuper (1995) 
could also fit, but seem to fit better with Arr. philinotis while his measurements for the epithet philonotis seem to fit better with our telmatiaea. The relatively good fit of spore measurements to one epithet may tempt one to consider conserving oniscus over telmatiaea, but the frequency of its use among this small selection of workers does not tell the whole story. Figure 8 shows that epithets other than oniscus have also been applied to species with spores matching those of Arr. telmatiaea: fusconigra (Bon 1997, Clémençon 1982; but not Bigelow 1985), icmadophila (Bigelow 1958, Redhead 1979), and epichysium (Lange \& Lange 1982). In addition, Fig. $8 \mathrm{~g}$ suggests that some collections called philonotis (Kuyper 1995, Lange \& Lange 1982) may be or have had an admixture of Arr. telmatiaea.

Of course, spore measurements alone are insufficient for species circumscription. Arrhenia telmatiaea is an obligatory sphagnophile. Our observations in this regard agree with the illustration of the type by Massee depicting basidiomata intimately attached to Sphagnum, and the several descriptions of Messrs Berkeley, Broome and Cooke, all stating that it occurs "in Sphagnum". Of the eight studies applying oniscus to a species whose spore measurements fit those of Arr. telmatiaea, only one (Gminder 2001) described it as an obligatory sphagnophile. Five (Favre 1948, Clémençon 1982, Bigelow 1985, Breitenbach \& Kränzin 1991, Elborne 2012) described it as a facultative sphagnophile, and two (Kuyper 1995, Bon 1997) stated that it grew with other mosses, but not with Sphagnum. Thus, clearly some species other than Arr. telmatiaea must have also been considered part of the taxon to which oniscus had been applied. The most likely candidate is Arr. philonotis, which shares its darker colour, smooth cap, liking for moorland in cold climates, and a large overlap of spore size, but not the obligatory relationship to Sphagnum.

Thus, despite the seeming frequency of using oniscus among leading workers, uniform nomenclatural-taxonomic consistency is lacking even in this small sampling. Using spore size as the only criterion, the epithet may be applied to the same species seven times, and possibly applied to another species once, while up to four other epithets may have been applied to the same species as well. Such conclusion produces major conflict with the reported ecology of the same entity, and vice versa. Using additional criteria make things worse, not better. For example, as mentioned earlier, consideration of cap ornamentation and texture produces additional confusion. In the case of Arr. telmatiaea the matter is confused even more by its later synonym, 0 . fusconigra, a smooth-capped species often misinterpreted as scaly. This problem caused Bigelow (1985) to err by synonymizing his correctly identified new scaly-capped taxon with this smooth-capped one, despite the difference in spore size and shape.

Because a) this lack of taxonomic-nomenclatural consistency among leading workers will cause significant change in past practice no matter what name is fixed, b) nomenclatural study has revealed that $\mathrm{Ag}$. oniscus is a later synonym for the current L. umbellifera, typified with that species (Voitk 2021), and c) there is available type material that fits the taxon without conflict, we elected to apply the earliest available sequence-confirmed name to this taxon. Selecting an optimal course becomes a matter of opinion. In our opinion, the extent of nomenclatural and taxonomic inconsistency argues against selecting any name shown to cause significant conflict with this group over Ag. telmatiaeus, whose sequenced type (along with the sequenced type of 0 . fusconigra) nestles in this clade. The name Agaricus telmatiaeus has been left largely unused in the past, partly because of initial confusion around the name, and lack of a type specimen in Kew (Dennis 1948), all of which relegated it to a nomen dubium (Legon \& Henrici 2005). Bigelow (1985) examined its type in NY, and recorded spore measurements that fit $O$. fusconigra. He stated these taxa should be compared, but because he was unable to confirm incrusted pigmentation, was uncertain about its correct placement. Examination of our own collections confirms that the species has incrusted pigment in cap hyphae (Fig. 7d). Applying a name that produces conflict with the original material is likely to become a potential focus for repeated future attempts at rectification. Should other workers consider a more frequently used epithet more advantageous, the Code provides a mechanism to conserve one name over another. Such legitimate conservation of a name over an optimally fitting one, via rules in place to serve the best interests of the users, should eliminate future temptation to change nomenclature far better than arbitrary selection of a name causing conflict at this time.

Page $32 / 45$ 
Similar inconsistency was observed with the three other species. Epithets with spore measurements fitting best with Arr. philonotis were philonotis (Favre 1948; Clémençon 1982; Bon 1997, Elborne 2008) and umbratilis (Lange \& Lange 1982). Bon described the species as an obligatory sphagniphile, suggesting he had another species in mind. Epithets with spore measurements fitting best with the two scaly-capped species were gerardiana (Bigelow 1958; Redhead 1979; Kuyper 1995; Elborne 2008), sphagnicola (Favre 1948; Clémençon 1982; Breitelbach \& Kränzlin 1991; Bon 1997), and fusca (Bigelow 1958).

In other words, past nomenclature has been inconsistent even in the hands of leading workers, by using multiple names for one species, by applying the same name to different species, and seemingly including more than one of the currently identified species under one name. Any nomenclatural reconciliation in this setting will result in some degree of instability in this small group of taxa.

In addition to the three obligate sphagnophiles, we identified Arr. philonotis as a facultative sphagnophile. Whether it interacts with Sphagnum, or merely shares with it a like for similar habitats is a matter for investigation. Molecular studies enabled us to name all other clades, leaving Arr. philonotis for the last. However, the application of the name was not by default: the light grey colour and scattered fine pileal hairs (Fig. 3e) described in the protologue enabled us to match this name to only one species. The type for Ag. philonotis, presumed lost, came from a bog close to Berlin, near the North Sea. The closest collection with good material that we sequenced came from a bog near BadenWürttemberg, Germany, a more midcontinental location in the foothills of the Alps, about $500 \mathrm{~m}$ asl. Although the biome differs slightly, we were reassured by finding some of our sequenced specimens in coastal barrens and moors similar to Lasch's collecting region. This decision seems to be supported by at least three major workers (Favre 1948; Kuyper 1995; Elborne 2008), who apply this epithet to collections with spore measurements similar to ours. That said, of the four, we know Arr. philonotis the least, and have only seen four collections of it in its habitat. Therefore, our descriptions (based only on sequenced specimens directly observed by us) should benefit from augmentation by future observations.

Finally, finding OPCUS, a facultative sphagnicolous species of Omphalina, was a pleasant surprise. Our study is focused on sphagnicolous arrhenias, but because OPCUS can be confused with them with ease, we present an informal description to alert the reader. Based on sequence studies to date, this is the only species of Omphalina in the NL. If there is a relationship with sphagnum, it is facultative, because the same species was encountered much more frequently in low-moss boreal grasslands on poor soil. Despite the reputed reddish colouration of omphalinas, macroscopic morphology was not always enough to separate some specimens of OPCUS (Fig. 5f, g) from Arr. philonotis (Fig. 3e; 5a, b), a non-blackish Arr. telmatiaea (Fig. 3f) or particularly from the more similar desquamated older Arr. gerardiana (Fig. 3d; 4f), a species that can appear reddish at times. However, OPCUS can be separated from these readily by spore size. Phylogenetic type studies of the Omphalina pyxidata complex to identify OPCUS, are outside the scope of the present study, but have been taken up by a colleague.

This experience demonstrates the major contribution of molecular analysis to taxonomy. When we rank organisms, we use their characters to separate them. Not all characters are equally relevant discriminators at all times.

Sometimes cap ornamentation, colour, spore size or ecology are excellent discriminators between species, while at other times some or all of these characters are irrelevant. Using molecular analysis to circumscribe the species in a group, permits retrospective examination, to learn which characters serve to separate these species and which do not. This requires familiarity with the organisms in their setting. A fresh comparison of protologues and original material to the characters of the identified clades will at times be rewarded with felicitous matches, which should bring about lasting stability to this relatively small group of interesting fungi. 


\section{Declarations}

\section{Ethics approval and consent to participate}

Not applicable.

\section{Consent for publication}

All co-authors have read the text and approve its publication, with implicit approval of the institutes where their work was carried out.

\section{Availability of data and materials}

All specimens are deposited in public fungaria/herbaria (Tabs 1-3), all sequences in publicly available gene depositories (Tabs 1-3), and all phylogenies in TreeBase (see text).

\section{Competing interests}

The authors have no relevant financial or other competing interests to disclose.

\section{Funding}

IS was supported by the Estonian Research Council (PRG1170), the European Regional Development Fund (Centre of Excellence EcolChange). EL was supported by Bridgewater College Faculty Development and Travel Funds.

\section{Authors' contributions}

AV and EL were primary contributors to study conception and design. Material preparation, data collection and analysis were performed by all authors. The first draft of the manuscript was written by AV and all authors commented on it and subsequent versions of the manuscript. All authors read and approved the final manuscript.

Code availability Not applicable.

\section{Acknowledgments}

AV is very grateful to Scott Redhead for many enlightening discussions about sphagnicolous and other omphalinoids. The authors owe an especial debt to Robert Lücking for work and spirited discussion as a collaborator of the earlier phases of this investigation. The authors are grateful to curators and collections managers of the fungaria $G B, H, K$, KR, M, MICH, NY, NYS, S, TAAM and TUF for the gifts and loans of specimens; to the curators of KR, NY, and NYS for illustrations and permission to use them. We also thank Gretchen Wade of Harvard Botany Libraries for help with fixing exact publication dates of old names, Teuvo Ahti for helping with botanical Latin interpretations and Irene Andersson, Jason Karakehian and Shaun Pennycook for help with finding obscure references, and discussions about taxonomy. We are particularly thankful to Scott Redhead and one anonymous reviewer of an earlier version for suggestions that led to this new report, as well as two anonymous reviewers for helpful suggestions to improve the current presentation. Authors are also grateful to the granting agencies for funding support, as indicated under Funding, above.

\section{References}


1. Abarenkov K, Nilsson RH, Larsson K-H, Alexander IJ, Eberhardt U, Erland S, Høiland K, Kjøller R, Larsson E, Pennanen T, Sen R, Taylor AFS, Tedersoo L, Ursing BM, Vrålstad T, Liimatainen K, Peintner U, Kõljalg U (2010a) The UNITE database for molecular identification of fungi-recent updates and future perspectives. New Phytol 186:281-285

2. Abarenkov K, Tedersoo L, Nilsson RH, Vellak K, Saar I, Veldre V, Parmasto E, Prous M, Aan A, Ots M, Kurin O, Ostonen I, Jõgeva J, Halapuu S, Põldmaa K, Toots M, Truu J, Larsson K-H, Kõljalg U (2010b) PlutoF-a Web based workbench for ecological and taxonomic research, with an online implementation for fungal ITS sequences.

Evolutionary Bioinf 6:189-196

3. von Albertini IB, Schweinitz von LD (1805) Conspectus fungorum in Luisatiae superioris agro Niskiensi crescentium. E Metodod Persooniana. Lipsiae

4. Berkeley MJ (1836) Fungi. In: The English Flora of Sir James Edward Smith. Class XXIV. Cryptogamia, vol. 5, part 2, Hooker JW (ed.). Longman, Rees, Orme, Brown \& Longman, London

5. Berkeley MJ, Broome CE (1865) XXXIV-Notices of British fungi. Ann Mag Nat Hist third series 15:313-322

6. Berkeley MJ, Broome CE (1883) XLII-Notices of British fungi. Ann Mag Nat Hist fifth series 12:370-374

7. Bigelow HE (1958) New species and varieties of Clitocybe from Michigan. Mycologia 50:37-51

8. Bigelow HE (1985) North American species of Clitocybe. Part II. Beih Nova Hedw Heft 81:281-473

9. Bjorbækmo MF, Carlsen T, Brysting A, Vralstad T, Høiland K, Ugland KI, Geml J, Schumacher T, Kauserud H (2010) High diversity of root associated fungi in both alpine and arctic Dryas octopetala. BMC Plant Biol 10:244

10. Bolton J (1788) An history of funguses growing about Halifax, vol 1. Brooks, Huddersfield, Halifax

11. Bon M (1997) Flore Mycologique d’Europe. Les Clitocybes, omphales et ressemblants. Doc Mycol, Mém hors série $4: 1-181$

12. Bresinsky A, Strangl J (1974) Beiträge zur Revision M. Britzelmayrs “Hymenomyceten aus Südbayern” 12. Weitere Tricholomataceen aus der Umgebung von Augsburg. Z für Pilzkunde 40:69-104

13. Breitenbach J, Kränzlin F (1991) Fungi of Switzerland, vol. 3. Boletes and Agarics, 1st part. Edition Mykologia, Lucerne

14. Britzelmayr M (1883) Dermini und Melanospori aus Südbayern. Bericht des Naturhistorischen Vereins in Augsburg 27:147-196

15. Brunner I, Frey B, Hartmann M, Zimmermann S, Gref F, Suz LM, Niskanen T, Bidartondo MI, Senn-Irlet B (2017) Ecology of alpine macrofungi-combining historical with recent data. Front Microbiol 8:1-13. https://doi.org/10.3389/fmicb.2017.02066

16. Buillard JBF (1872) Agaricus tigrinus. Herbier de la France 2:pl. 49-96

17. Bunyard BA, Wang Z, Malloch D, Clayden S, Voitk A (2008) New North American records of Ascocoryne turficola (Ascomycota: Heliotales). Fungi Mag 1(2):23-31

18. Cooke MC (1871) Handbook of British fungi. McMillan \& Co, London

19. Cooke MC (1881-1883) Illustrations of British fungi (Hymenomycetes), vol. 2.Williams and Norgate, London

20. Cooke MC (1883) Handbook of British fungi, Second and revised edition. Grevillea (supplement), London

21. Cooke MC (1884) New British fungi. Grevillea 12(63):6-70

22. Clémençon H (1982) Kompendium der Blätterpilze Europäische omphalinoide Tricholomataceae. Zeitschr Mykol 48:195-237

23. Dennis RWG (1948) Some little-known British species of Agaricaceae. Trans Brit Mycol Soc 31:191-209 
24. Elborne SA (2008) Arrhenia. In: Knudsen H, Vesterholt J (eds) Funga Nordica. Agaricoid, Boletoid and Cyphelloid Genera, Nordsvamp, Copenhagen pp 226-234

25. Elborne SA (2012) Arrhenia. In: Knudsen H, Vesterholt J (eds) Funga Nordica. 2nd. ed. Agaricoid, Boletoid, Clavarioid, Cyphelloid and Gastroid Genera, Nordsvamp, Copenhagen pp 252-260

26. Favre J (1948) Matériaux pour la Flore Cryptogramique Suisse, vol 10, fasc. 3. Büchler \& Cie, Berne

27. Favre J (1955) Les champignons supérieures de la zone alpine du Parc National Suisse. Ergebnisse der Wissenschaftlichen Untersuchungen des Schweizerischen Nationalparks 5:1-212

28. Fries EM (1818) Observationes mycologicæ præcipue ad illustrandam floram Suecicam, vol 2. Gerhard Bonnier, Copenhagen

29. Fries EM (1821) Systema mycologicum vol 1. Berlingiana, Lund

30. Fries EM (1849) Summa vegetabilium Scandinaviæ, vol 2. A. Bonnier, Uppsala

31. Fries EM (1867) Monographia Hymenomycetum Sueciae, vol 1. Leffler, Uppsala

32. Geml J, Timling I, Robinson CH, Lennon N, Nusbaum HC, Brochmann C, Noordeloos ME, Taylor DL (2012) An arctic community of symbiotic fungi assembled by long-distance dispersers: phylogenetic diversity of ectomycorrhizal basidiomycetes in Svalbard based on soil and sporocarp DNA. J Biogeogr 39:74-88

33. Gminder A (2001) Omphalina. In: Kriegelsteiner GJ (ed) Die Grosspilze Baden-Württembergs. Band 3. Ständerpilze, Blätterpilze 1 Ulmer, Stuttgart pp 476-489

34. Gouy M, Guindon S, Gascuel O (2010) SeaView version 4: a multiplatform graphical user interface for sequence align- ment and phylogenetic tree building. Mol Biol Evol 27:221-224

35. Katoh K, Standley DM (2013) MAFFT multiple sequence alignment software version 7: improvements in performance and usability. Mol Biol Evol 30:722-780

36. Kuyper TW (1987) Omphalina.. In: Bas C, Kuyper TW, Noordeloos ME, Vellinga EC (eds) Flora Agaricina Neerlandica, vol 3. A.A. Balkema, Rotterdam, pp 78-88

37. Kuyper TW (1995) Omphalina.. In: Bas C, Kuyper TW, Noordeloos ME, Vellinga EC (eds) Flora Agaricina Neerlandica, vol 3. A.A. Balkema, Rotterdam, pp 78-88

38. Lange M, Lange B (1982) Agarics growing in Sphagnum: specialization and distribution in arctic and alpine zones. In: Laursen GA, Ammirati JF (eds.) Arctic and alpine mycology. The first international symposium on arctoalpine mycology. Barrow, Alaska, 16-23 August, 1980 University of Washington Press, Seattle pp 150-163

39. Lasch WG (1828) Enumeratio agaricorum marchiae Brandenburgicae, nondum in floris nostratibus nominatorum, cum observationibus in cognitos et novorum descriptionibus. Linnaea 3:378-430

40. Legon NW, Henrici A (2005) Checklist of the British \& Irish Basidiomycota. Royal Botanic Gardens. Richmond

41. Lodge JD, Mahajabeen P, Matheny PB, Aime MC, Cantrell SA, Boertmann D, Kovalenko A, Vizzini A, Dentinger BTM, Kirk PM et al (2014) Molecular phylogeny, morphology, pigment chemistry and ecology in Hygrophoraceae (Agaricales). Fungal Diver 64:1-99

42. Lickey EB, Hughes KW, Petersen RH (2003) Phylogenetic and taxonomic studies in Artomyces and Clavicorona (Homobasidiomycetes; Auriscalpiaceae). Sydowia 55:181-254

43. Lutzoni F (1997) Phylogheny of lichen- and non-lichen-forming mushrooms and the utility of testing for combinability among multiple data seta. Syst Biol 46:373-406

44. Orton PD (1960) New check-list of British Agarics and Boleti, part 3: Notes on genera and species in the list. Trans Brit Mycol Soc 43:159-439 
45. Osmundson TW, Robert VA, Schoch CL, Baker LJ, Smith A, Robich G, Mizzan L, Garbelotto MM (2013) Filling gaps in biodiversity knowledge for macrofungi: contributions and assessment of an herbarium collection DNA barcode sequencing project.PLoS ONE8 (4), E62419

46. Peck CH (1873) ["1873-1874"] Descriptions of new species of fungi. Bull Buffalo Soc Nat Sci 1:41-72

47. Persoon CH (1794) Neuer Versuch einer systematischen Eintbeilung der Schwämme. Neues Magazin für die Botanik in ihrem ganzen Umfange 1:63-128

48. Persoon CH (1801) Synopsis Methodica Fungorum, vol 2. Henricus Dieterich, Göttingen

49. Ronquist F, Teslenko M, Mark P, van der, Ayres DL, Darling A, Höhna S, Larget B, Liu L, Suchard MA, Huelsenbeck JP (2012) MrBayes 3.2: efficient Bayesian phylogenetic inference and model choice across a large model space. Syst Biol 61:539-542

50. Redhead SA (1979) A study of the basidiomycetes in the eastern sections of the Canadian boreal forest. PhD Thesis. University of Toronto

51. Saar I, Voitk A (2015) Type studies of two Tricholomompsis species described by Peck. Mycol Progress 14:46

52. Stamatakis A (2014) RAxML version 8: a tool for phylogenetic analysis and post-analysis of large phylogenies. Bioinformatics 30:1312-1313

53. Stefleu FA, Cowan RS (1976) Taxonomic Literature, 2nd edn. Bohn, Scheltema \& Holkana, Utrecht

54. Sulzbacher MA, Wartchow F, Ovrebo CL, Sousa JO, Baseia IG, Moncada B, Lücking R (2016) Sulzbacheromyces caatingae: notes on its systematics, morphology and distribution based on ITS barcoding sequences.

Lichenologist 48:61-70

55. Thiers B (2021), continuously updated Index Herbariorum: a global directory of public herbaria and associated staff. New York Botanical Garden's Virtual Herbarium http://sweetgum.nybg.org/ih/. (Last accessed Feb. 25, 2021)

56. Turland NJ, Wiersema JH, Barrie FR, Greuter W, Hawksworth DL, Herendeen PS, Knapp S, Kusber W-H, Li D-Z, Marhold K, May TW, McNeill J, Monro AM, Prado J, Price MJ, Smith GF (eds) (2018) International Code of Nomenclature for algae, fungi, and plants (Shenzhen Code) adopted by the Nineteenth International Botanical Congress Shenzhen, China, July 2017. Regnum Vegetabile 159. Koeltz Botanical Books, Glashütten. DOI https://doi.org/10.12705/Code.2018

57. Vašutová M, Dvořák D, Beran M (2013) Rare macromycetes from raised bogs in the Hrubý Jeseník Mts. (Czech Republic). Czech Mycol 65:45-67

58. Voitk A (2021) Typification of Agaricus cespitosus, A. oniscus, and A. sphagnicola and their synonymy with Lichenomphalia umbellifera.Mycotaxon136 (in press).

59. Voitk A, Burzynski M (2018) Great Caribou Island Arrhenia quest. Omphalina 9(8):14-18

60. Voitk A, Saar I, Lücking R, Moreau P-A, Corriol G, Krisai-Greilhuber I, Thorn RG, Hay CRJ, Moncada B, Gulden G (2020) Surprising morphological, ecological and ITS sequence diversity in the Arrhenia acerosa complex (Basidiomycota: Agaricales: Hygrophoraceae). Sydowia 73:133-162

61. Vu D, Groenewald M, de Vries M, Gehrmann T, Stielow B, Eberhardt U, Al-Hatmi A, Groenewald JZ, Cardinali G, Houbraken J, Boekhout T, Crous PW, Robert V, Verkley GJM (2019) Large-scale generation and analysis of filamentous fungal DNA barcodes boosts coverage for kingdom fungi and reveals thresholds for fungal species and higher taxon delimitation. Stud Mycol 92:135-154

62. Zvyagina EA, Alexandrova AV, Bulyonkova TM (2015) Omphalina discorosea: taxonomical position of the species. Micologia i Phitopathologia 49:19-25 


\section{Figures}

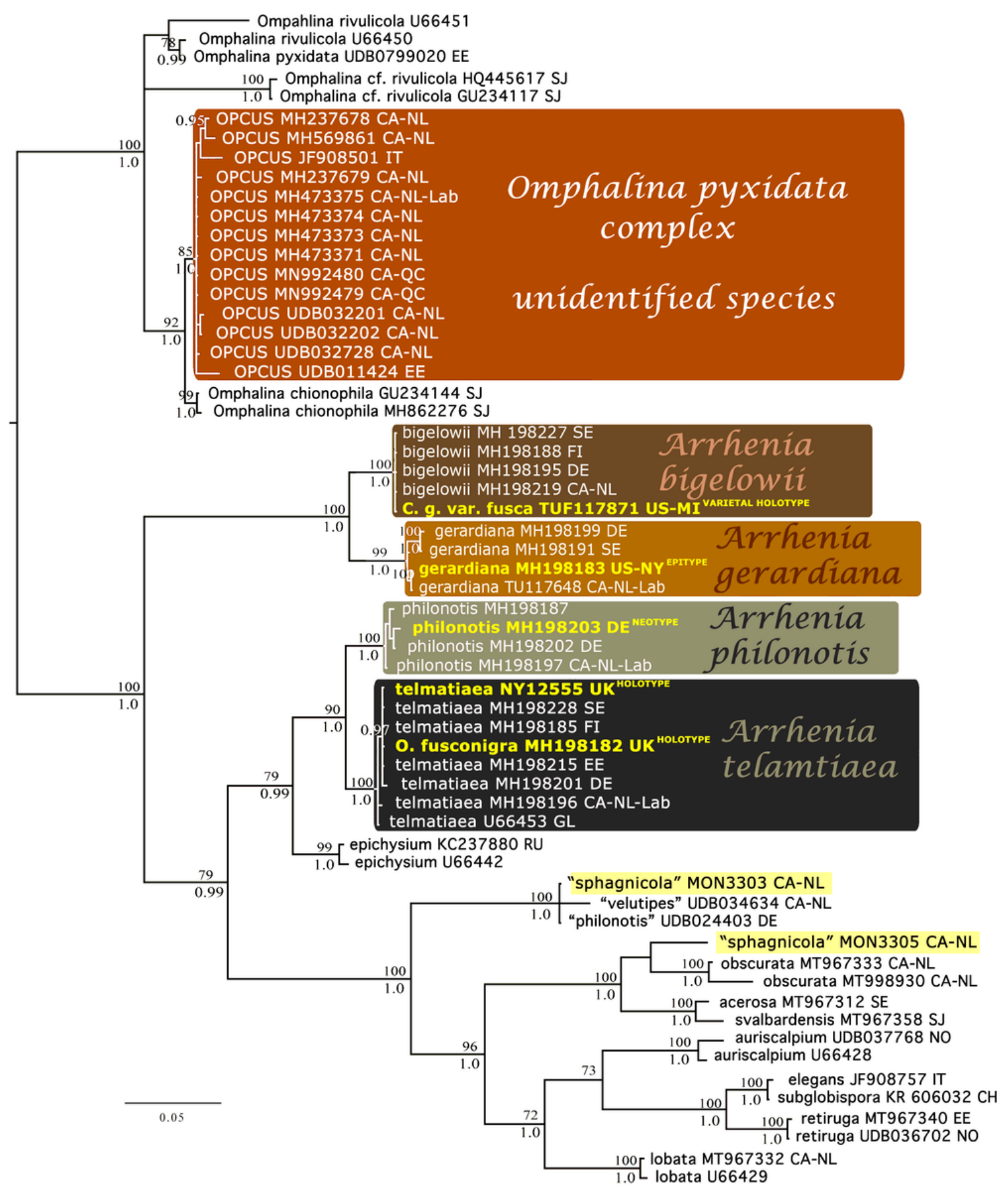

\section{Figure 1}

Placement of the sphagnicolous arrhenias within Arrhenia, and OPCUS (as "Omphalina cf. pyxidata") within Omphalina. Note the unidentified singletons, "sphagnicola", collected as presumptive sphagnicolous arrhenias, but not treated here due to lack of data. ML bootstrap support $\geq 70 \%$ and the Bayesian posterior probabilities $\geq 95 \%$ are shown above and below the branches (bs/pp), respectively 


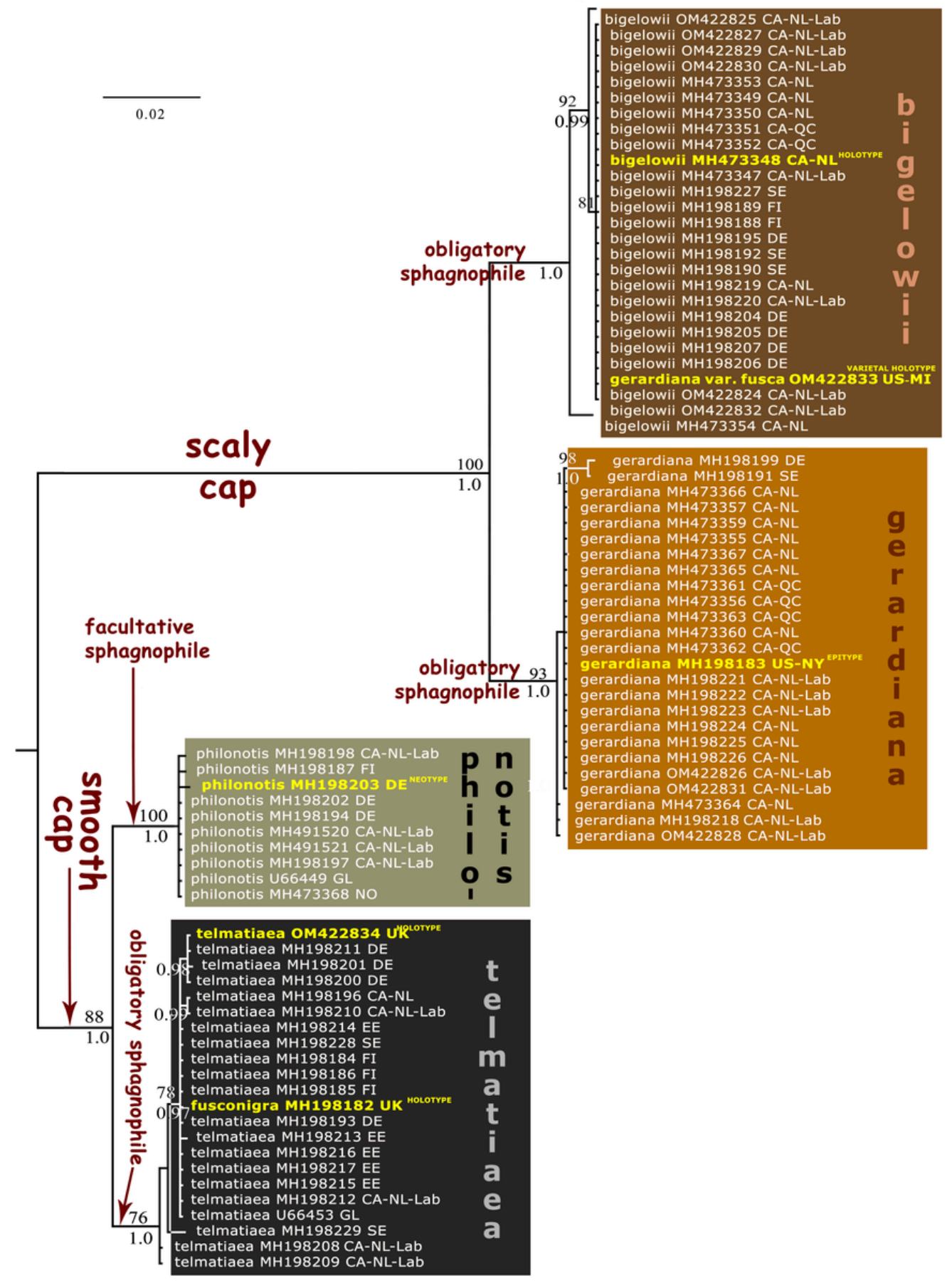

Figure 2

Best-scoring tree focusing on the four formally accepted sphagnicolous arrhenias, based on maximum likelihood analysis of the ITS barcoding marker. Country (plus province/state for US and CA) identified by ISO Alpha 2 codes. "Lab" marks NL specimens from Labrador. Types shown in bold yellow. Specimens identified by current names, except types, identified by the original epithets. All sequences were generated for this study, except U66449 and U66453, the only deposits in GenBank from this group when we began this investigation. ML bootstrap support $\geq 70 \%$ and the Bayesian posterior probabilities $\geq 95 \%$ are shown above and below the branches (bs/pp), respectively 


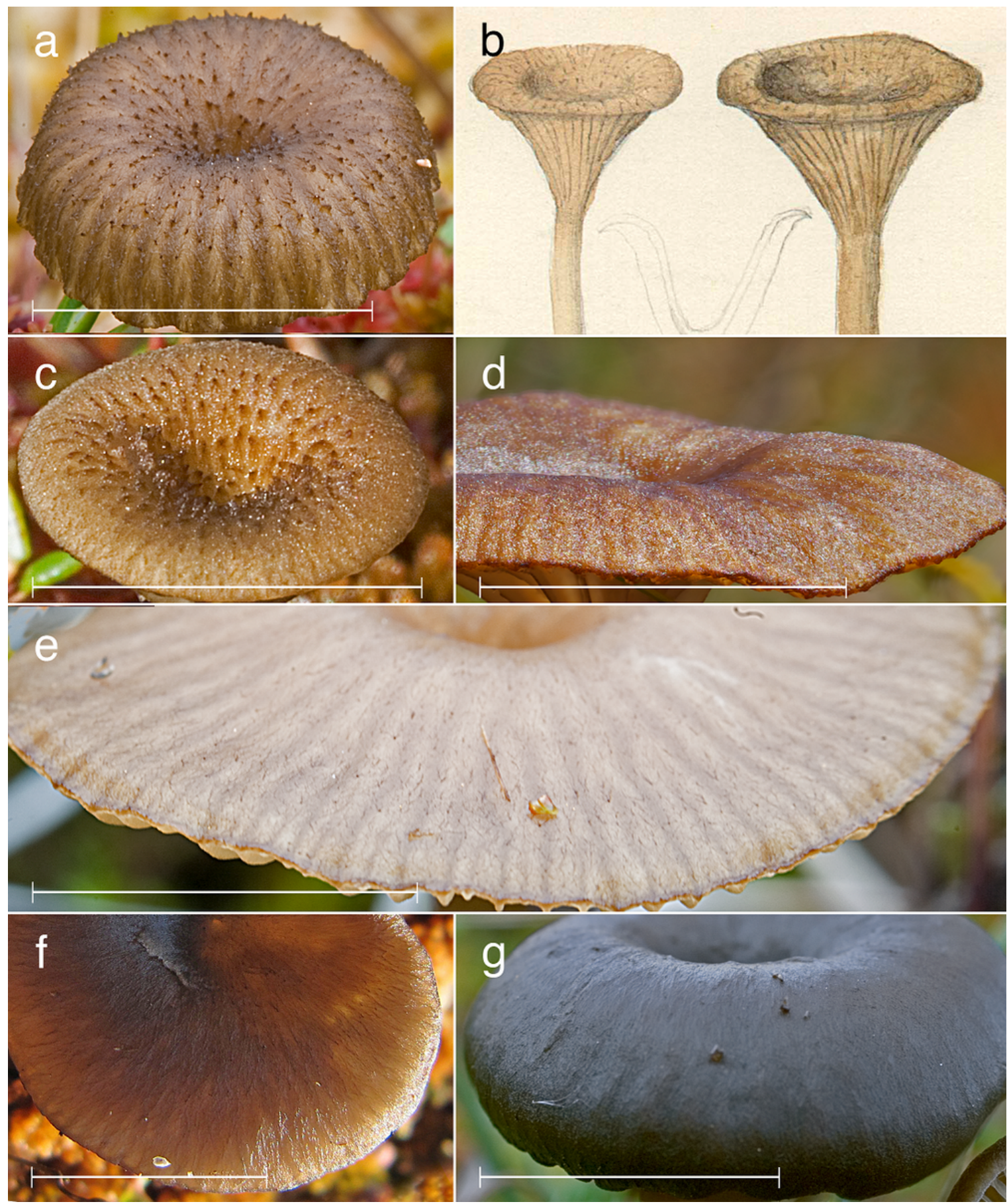

Figure 3

Cap texture of sphagnicolous arrhenias. Bars $=10 \mathrm{~mm}$. a. The dramatically scaly cap of Arr. bigelowii (08.08.12.av05), unlikely to be described by diminutives, "sparsely wooly" or "minutely squamulose". b. Peck's aquarelle of his Ag. gerardianus with evident scales (photo courtesy NYS). c. The equally obvious scaly cap of Arr. gerardiana (05.07.03.av02). d. The smooth cap of a very mature desquamated Arr. gerardianai (17.07.05.av05), making identification difficult. e. Cap of Arr. philonotis (KL-015) showing subtle "scale pattern" embedded in the pileipellis, occasionally elevated to create a scattering of very fine hairs. f. A brownish and lighter cap of Arr. telmatiaea (GNP-064). A pattern resembling the radial scales of Arr. bigelowii or Arr. gerardiana can be seen, but these remain entirely adpressed in the pileipellis, with no attempts of the tips to rise. g. Cap of a very dark Arr. telmatiaea (05.09.08.av02). The adpressed "scale pattern" in the pileipellis is present, although not as evident 


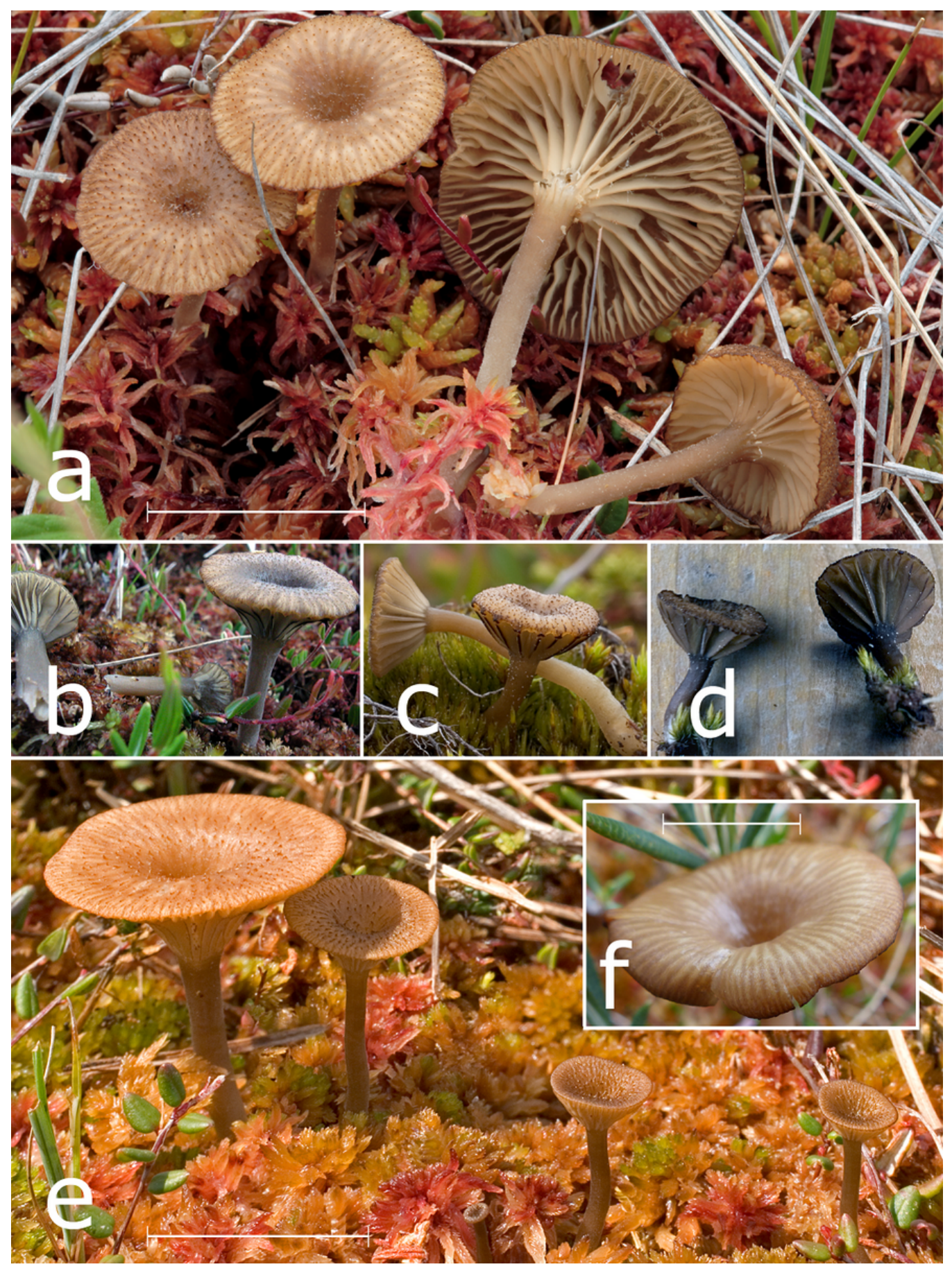

\section{Figure 4}

Scaly-capped sphagnicolous arrhenias of NL in situ. Bars $=10 \mathrm{~mm}$. a. Arrhenia bigelowii (07.06.26.av01). b. The darkening reaction, occasionally seen with Arr. bigelowii. Collection 04.07.05.av03, the type specimen and model for the FNL logo. c. Collection 05.06.15.av01: in situ appearance at the time of collection. d. Appearance when taken from collecting basket, ca. 3 hours later-the most dramatic darkening reaction of Arr. bigelowii we have observed. E. Arrhenia gerardiana (06.06.15.av04)-macroscopically indistinguishable from Arr. bigelowii. f. (insert; photo: Michael Burzynski) A large overmature specimen (17.07.04.av02) with scales no longer evident (see also Fig. 3d) 


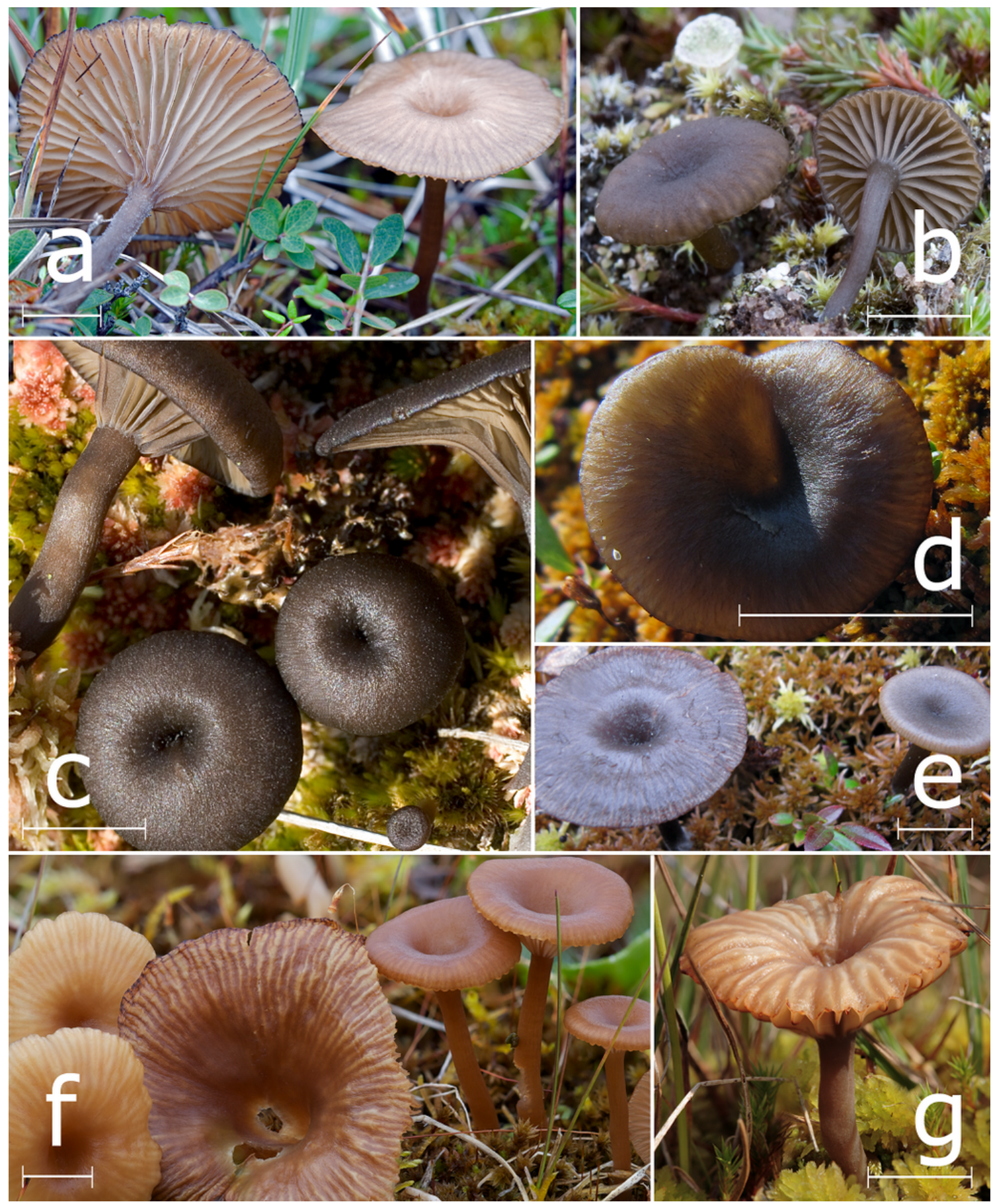

Figure 5

Smooth-capped sphagnicolous omphalinoids of NL. Bars = $10 \mathrm{~mm}$. a. Arrhenia philonotis (KL-015), "normal” light colouring. b. Arrhenia philonotis (LS-008), dark colouring. c. Arrhenia telmatiaea (05.09.08.av02). The near-black dark brown colour readily distinguishes this species from the others most of the time. d. Arrhenia telmatiaea (GNP-064). Lighter brown. e. Arrhenia telmatiaea (16.09.14.av01) medium dark caps. Colours of caps as on photos d and e might be confused with other species. f. OPCUS (16.10.18.av01) from unfertilized and mossy grassland, revealing full spectrum of fruiting body appearance. This species and Arr. philonotis are both larger (mature cap diameter $>25 \mathrm{~mm}$ ), smooth-capped, and brown. The reddish tones of Omphalina and the greyish tones of Arrhenia are not always as obvious as on these photos. g. OPCUS (07.07.24.av01) growing in Sphagnum 

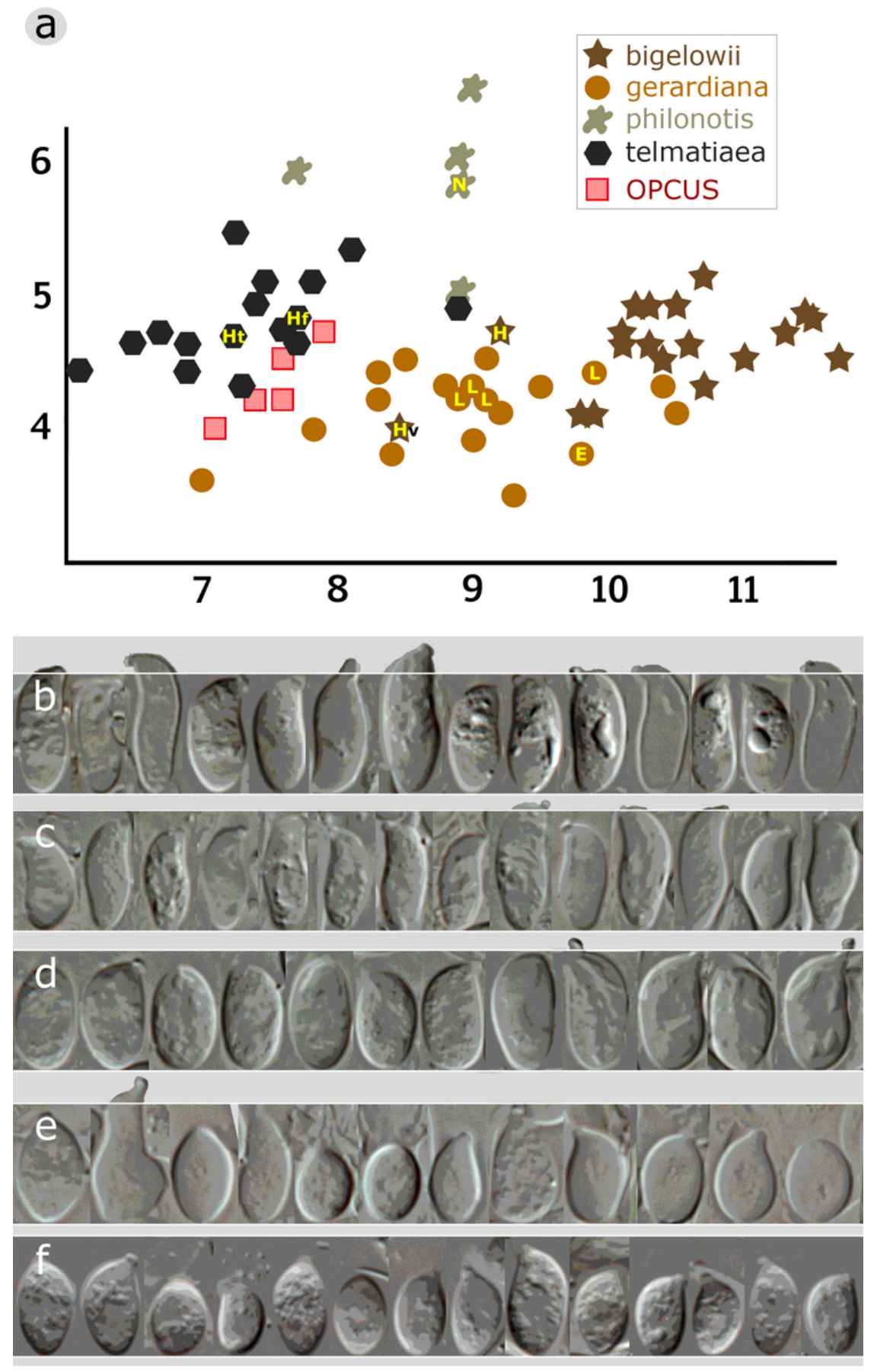

\section{Figure 6}

Basidiospores of studied species. a. Average spore sizes of sequence-identified sphagnicolous omphalinoids, length in $\mu \mathrm{m}$ on $\mathrm{x}$-axis, width on $\mathrm{y}$-axis. $\mathrm{H}=$ holotype; $\mathrm{Hv}=$ varietal holotype; $\mathrm{Ht}=$ holotype of $\mathrm{Ag}$. telmatiaeus, $\mathrm{Hf}=$ holotype of $O$. fusconigra; $\mathrm{N}=$ neotype; $\mathrm{L}=$ lectotype (4 basiomata, not sequenced); $\mathrm{E}=$ epitype. $\mathrm{b}-\mathrm{f}$. basidiospores of the studied species. Upper white line marks $10 \mu \mathrm{m}$. b. Arr. bigelowii, c. Arr. gerardiana, d. Arr. philonotis, e. Arr. telmatiaea, f. OPCUS. 


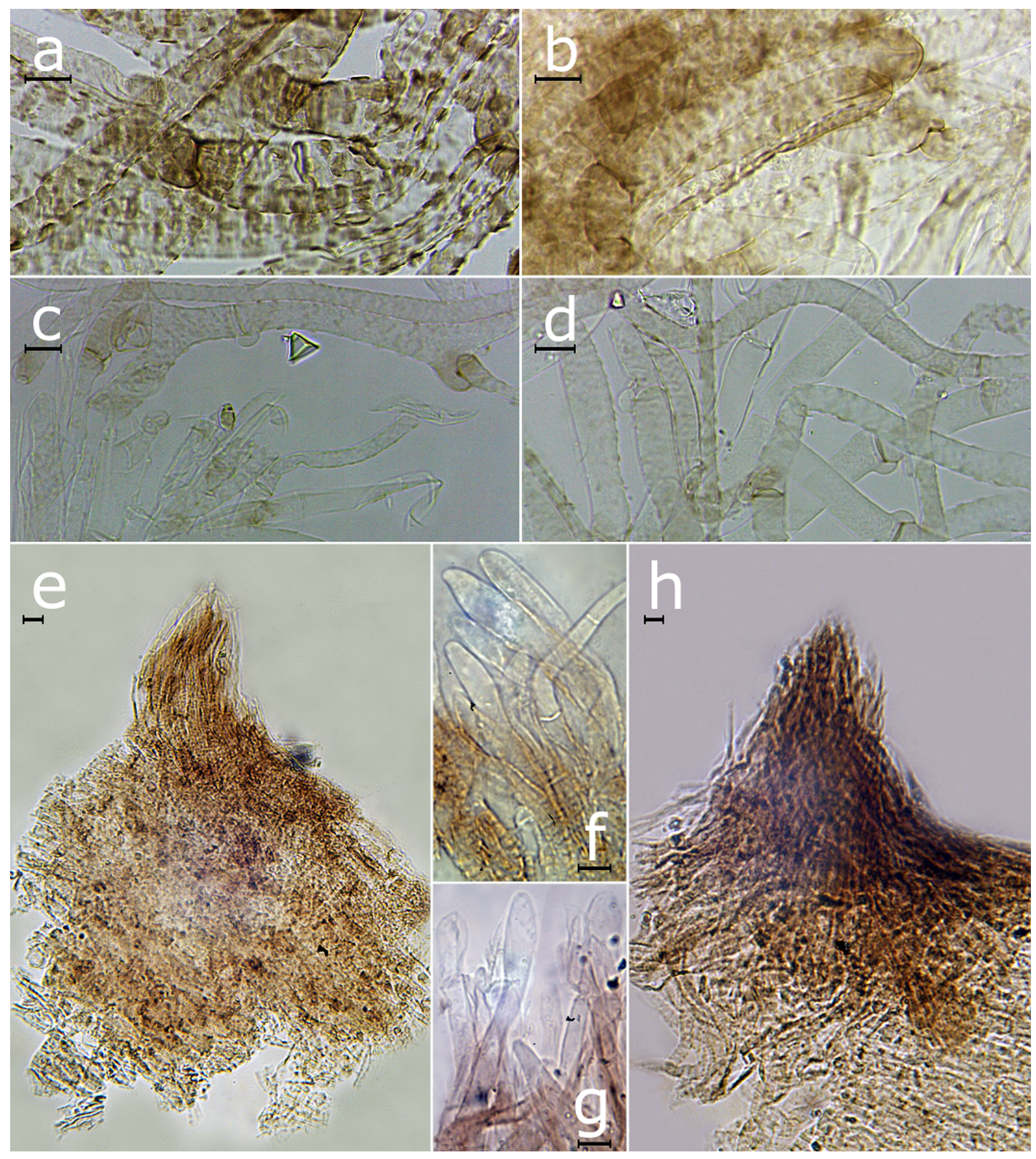

Figure 7

Cap micromorphology. Scale bars $10 \mu \mathrm{m}$. a. Incrusted pigment Arr. bigelowii (08.08.12.av05). b. Incrusted pigment Arr. gerardiana (17.07.05.av05). c. Incrusted pigment Arr. philonoitis (KL-015). d. Incrusted pigment Arr. telmatiaea (05.09.08.av02), the same specimen as Fig. 3e, from the collection on Fig. 5c. e. Scales of Arr. bigelowii (10.08.17.av01). b. Scales of Arr. gerardiana (10.07.13.av06) 


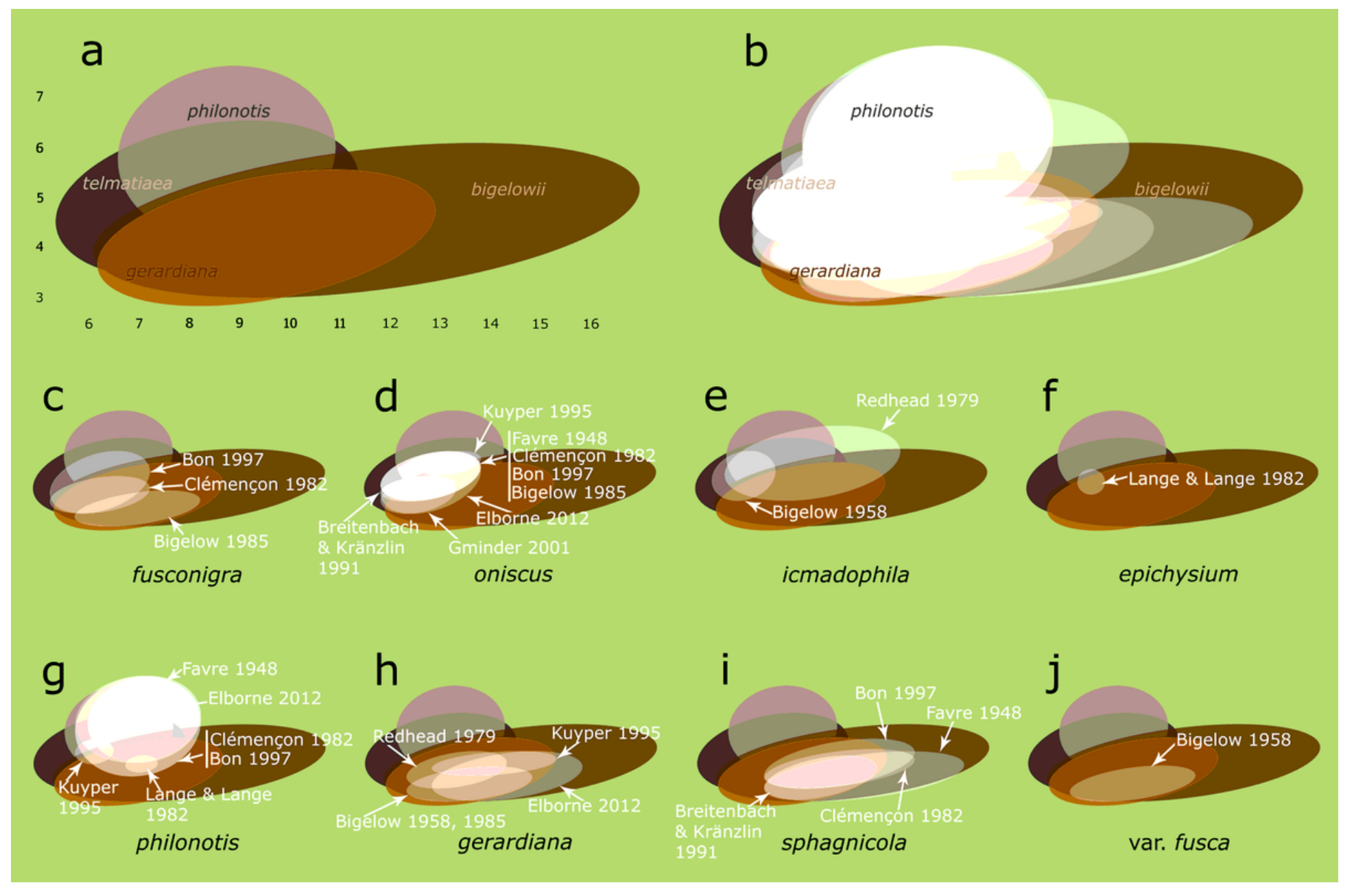

\section{Figure 8}

Comparison of spore measurements of our four species, with those reported in the ten major publications cited in the Introduction. a. Measurement as for Fig. 6. Ranges for our four species. b. Combined ranges of all species reported by all cited authors, represented by translucent white ovals superimposed on our results. $\mathbf{c}-\mathbf{j}$. Ranges for each epithet used by the cited authors on each separate view, again superimposed on our measurements. The ranges recorded by Lange \& Lange (1982) are for average values of the collections, not the complete range. 